\title{
Development and characterization of an electron gun for ultrafast electron microscopy
}

\author{
DisSERTATION \\ zur Erlangung des mathematisch-naturwissenschaftlichen Doktorgrades \\ "Doctor rerum naturalium" \\ der Georg-August-Universität Göttingen \\ im Promotionsprogramm ProPhys \\ der Georg-August University School of Science (GAUSS)
}

vorgelegt von

Herrn Dipl.-Phys. Reiner Bormann

aus Jena

Göttingen, 2015 
Betreuungsausschuss

Prof. Dr. Claus Ropers IV. Physikalisches Institut

Georg-August-Universität Göttingen

Prof. Dr. Rainer G. Ulbrich IV. Physikalisches Institut

Georg-August-Universität Göttingen

Mitglieder der Prüfungskommission

Referent: Prof. Dr. Claus Ropers, IV. Physikalisches Institut, Georg-August-Universität Göttingen

Korreferent: Prof. Dr. Markus Münzenberg, Institut für Physik, Ernst-Moritz-Arndt Universität Greifswald

Weitere Mitglieder der Prüfungskommission:

Prof. Dr. Konrad Samwer I. Physikalisches Institut, Georg-August-Universität Göttingen

Prof. Dr. Christian Jooß Institut für Materialphysik, Georg-August-Universität Göttingen

Prof. Dr. Wolfram Kollatschny Institut für Astrophysik, Georg-August-Universität Göttingen

Prof. Dr. Rainer G. Ulbrich IV. Physikalisches Institut, Georg-August-Universität Göttingen

Tag der mündlichen Prüfung: 27.11.2015 


\section{Abstract}

Ultrafast electron microscopy is an emerging field of research offering the potential to investigate structural processes on a femtosecond time-scale with nanometer resolution. In order to further advance ultrafast electron microscopes, an improvement of the electron gun is necessary with respect to spatial and temporal beam properties as well as the electron yield. In this thesis, photoemission characteristics of laser-driven nanotip cathodes incorporated into a Schottky-type emitter assembly are experimentally and theoretically investigated. Specifically, electron trajectories, emission patterns, transverse beam emittances and electron pulse durations are analyzed with respect to the emission site and settings of the electron gun geometry. Two operation regimes are identified offering high magnification or a large electron count rate. In the last part of this work, measurements of the electron beam quality (emittance, spot size, beam profile, electron yield) are conducted using a laser-driven needle emitter in a transmission electron microscope. 



\section{Contents}

1 Introduction $\quad 1$

2 Methods and concepts $\quad \mathbf{5}$

2.1 UTEM concept . . . . . . . . . . . . . . . . . . 5

2.2 Conventional electron sources . . . . . . . . . . . . . . . . 7

2.3 Pulsed electron sources . . . . . . . . . . . . . . . . . . . . . . . 14

2.4 Electron beam characteristics . . . . . . . . . . . . . . 21

2.5 Fundamentals in electron microscopy . . . . . . . . . . . . . . . . . 27

3 Prerequisites for simulation $\quad 35$

3.1 Numerical simulation method . . . . . . . . . . . . . . . . 35

3.2 Electrostatic landscape and $\Gamma$-parameter . . . . . . . . . . . . . 41

4 Experimental setup and first measurements $\mathbf{4 5}$

4.1 Fabrication of sharp tungsten tips . . . . . . . . . . . . . . . 46

4.2 Gun assembly alignment . . . . . . . . . . . . . . . . . . 47

4.3 Experimental setup . . . . . . . . . . . . . . . . . . . 47

4.4 Laser focus size . . . . . . . . . . . . . . . . . . . . . 50

4.5 Photocurrent measurement . . . . . . . . . . . . . . 51

4.6 Nonlinearity of the photoemission process . . . . . . . . . . . . . 52

4.7 Field emission . . . . . . . . . . . . . . . . . . . . 53

5 Spatial distribution of photoemitted electrons $\quad 57$

5.1 Electron trajectories . . . . . . . . . . . . . . . . . . 57

5.2 Emission patterns . . . . . . . . . . . . . . . . . . 62

5.3 Maps of electron emission sites . . . . . . . . . . . . . . . . . 71

5.4 Polarization dependence . . . . . . . . . . . . . . . . . . 75

$\begin{array}{llr}6 & \text { Gun performance capabilities } & \mathbf{7 9}\end{array}$

6.1 Transmission . . . . . . . . . . . . . . . . . . . 79

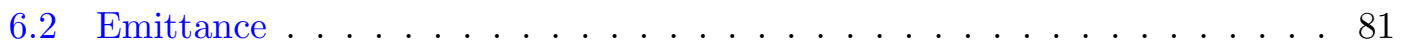

6.3 Temporal properties . . . . . . . . . . . . . . . 83

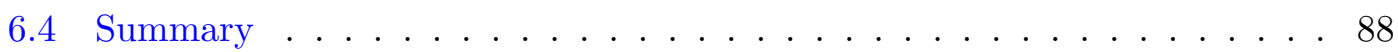

$\begin{array}{llr}7 & \text { UTEM characterization } & \mathbf{8 9}\end{array}$

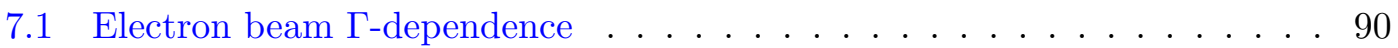

7.2 Electron beam properties . . . . . . . . . . . . . . . . 91

7.3 Electron beam pattern within the TEM . . . . . . . . . . 96 
8 Conclusions $\quad 99$

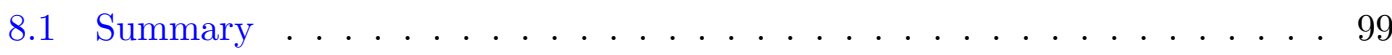

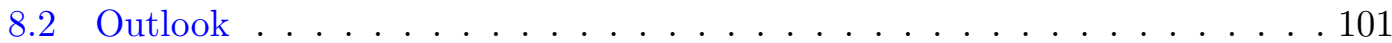

$\begin{array}{ll}\text { A Abbreviations and symbols } & 105\end{array}$

$\begin{array}{ll}\text { Bibliography } & 109\end{array}$

$\begin{array}{lr}\text { Publications and Conferences } & 127\end{array}$

Acknowledgments

129 


\section{Chapter 1}

\section{Introduction}

Mapping the dynamics of complex nanoscopic systems upon ultrafast ${ }^{1}$ excitation requires novel approaches in instrumentation. For a variety of physical and chemical questions, such as structural dynamics in chemical reactions, energy transfer in inhomogeneous systems, or phase transitions in layered bulk materials and at surfaces, the ability to observe ultrafast non-equilibrium processes on a nanometer length-scale is necessary. Several techniques are used to study ultrafast dynamics, such as x-ray spectroscopy, diffraction and crystallography [1-5], or electron diffraction and microscopy [6-21]. Xray sources are best suited for single-shot experiments and studies of large proteins with high resolution [19, 22]. In comparison, electron sources allow for studying thin samples in diffraction and imaging, and can be realized as a tabletop setup. In order to measure spatio-temporal dynamics in heterogeneous structures, at interfaces or defects, an electron microscope is beneficial providing both, high temporal and high spatial resolution.

For ultrafast electron microscopy, an essential part is the source which generates ultrashort electron pulses. The most common method of generating ultrafast electron pulses is illuminating a flat photocathode by femtosecond laser pulses [7, 14-16, 18, 23-25]. However, the spatial coherence of the resulting electron pulse is limited due to the fact that electron emission occurs over the size of the laser focus, which is typically on the order of several tens of micrometers. In comparison, the high coherence of conventional electron sources stems from the small source size in combination with filtering electrons,

\footnotetext{
${ }^{1}$ Here, ultrafast refers to sub-nanosecond time-scales.
} 
which are emitted at high angles with respect to the optical axis. For time-resolved electron microscopy, the generation of ultrafast electron pulses with high coherence is essential as well. However, blocking the majority of photoemitted electrons is not an option, since the number of generated electrons is usually very low and a minimum number of at least $10^{6}-10^{7}$ electrons is necessary in order to obtain an image [26, 27]. Therefore, an optimization of the electron gun with respect to various beam parameters is required.

In this thesis, photoemitted electrons from a nanotip incorporated into a Schottky-type electron gun assembly are characterized in terms of spatial and temporal beam properties. The utilization of such an needle emitter provides the high spatial coherence known from conventional electron microscopes. Preliminary work has proven promising characteristics for this approach [28-31]. Using the inhomogeneous field distribution and electric field enhancement at the apex of such a tip, in combination with nonlinear photoemission, offers the potential of an electron source yielding simultaneously high spatial and temporal resolutions [17, 28, 31-34]. Very recently, such a nanotip was successfully integrated into an ultrafast low-energy electron diffraction (ULEED) setup [17, 35]. The present work, on the other hand, will deal with an electron source for ultrafast transmission electron microscopy (UTEM). The first set of experiments is conducted in a prototype vacuum chamber using an identical electron gun assembly as installed in the UTEM. Spatial electron distributions are characterized by means of different voltages applied to the individual gun electrodes as well as varying optical illumination conditions. Ultimately, the electron gun is transferred into a functional TEM, and first measurements are presented in this work. Beam parameters such as the emittance and brightness are quantitatively characterized. The results from the prototype chamber are used to correctly position the laser pulse and set the electrode voltages, as this is crucial in order to obtain the beam characteristics needed for high-quality imaging. In addition, electron trajectories are simulated and used to analyze the system performance over a broad parameter range, in order to find optimum conditions. In the outlook, suggestions for future improvements are presented, and alternative approaches to nonlinear photoemission for generating ultrafast electron pulses are discussed.

The thesis is structured as follows. Chap. 2 gives an introduction into the methods and concepts of (ultrafast) electron microscopy, as well as a brief introduction into fundamentals for electron beam characterization. In Chap. 3, the numerical simulation method 
and the electrostatic landscape within the electron gun is described. Chap. 4 explains the experimental setup and the fabrication of sharp tungsten tips. Furthermore, preliminary measurements concerning the nonlinearity of the photoemission process or field emission are presented. The spatial distribution of photoemitted electrons measured in the prototype chamber is addressed in Chap. 5, including simulated results. Subsequently, Chap. 6 shows characteristics of the electron gun continuing the results presented in Chap. 5. In Chap. 7, measurements concerning the electron beam parameters are conducted in a transmission electron microscope using the ultrafast electron gun. 



\section{Chapter 2}

\section{Methods and concepts}

This chapter addresses to the fundamentals in ultrafast electron microscopy. First, the concept of an ultrafast electron microscope is outlined. Subsequently, conventional (Sec. 2.2) and pulsed (Sec. 2.3) electron sources are described including the underlying emission processes. Next, quantities are introduced, which are used in order to characterize an electron beam (Sec. 2.4). At the end of the chapter (Sec. 2.5), the fundamental setup of a transmission electron microscope is described.

\subsection{The concept of an ultrafast electron microscope}

Electron microscopes nowadays allow for the investigation of materials on the atomic scale. However, a temporal resolution is at most given by the video frame rate of the camera system. The limit for standard electron microscope cameras maintaining atomic spatial resolution is typically in the range of $10-60$ frames per second $(\mathrm{fps})^{1}$, occasionally as high as $1600 \mathrm{fps}^{2}$. Certainly, the time resolution is still far away from the pico- to femtosecond regime required for studying structural or electronic dynamics [27, 36].

The basic idea of an ultrafast electron microscope is rather intuitive. By replacing the static electron gun with a pulsed electron source, high temporal resolution can

\footnotetext{
${ }^{1}$ For example Direct Electron DE-16 (120fps, http://www.directelectron.com/ documents/Specs-DE16.pdf) or Gatan UltraScan (30fps, http://www.gatan.com/products/ tem-imaging-spectroscopy/ultrascan-camera)

${ }^{2}$ Gatan K2 IS (400 fps, http://www.gatan.com/products/tem-imaging-spectroscopy/k2-camera)
} 


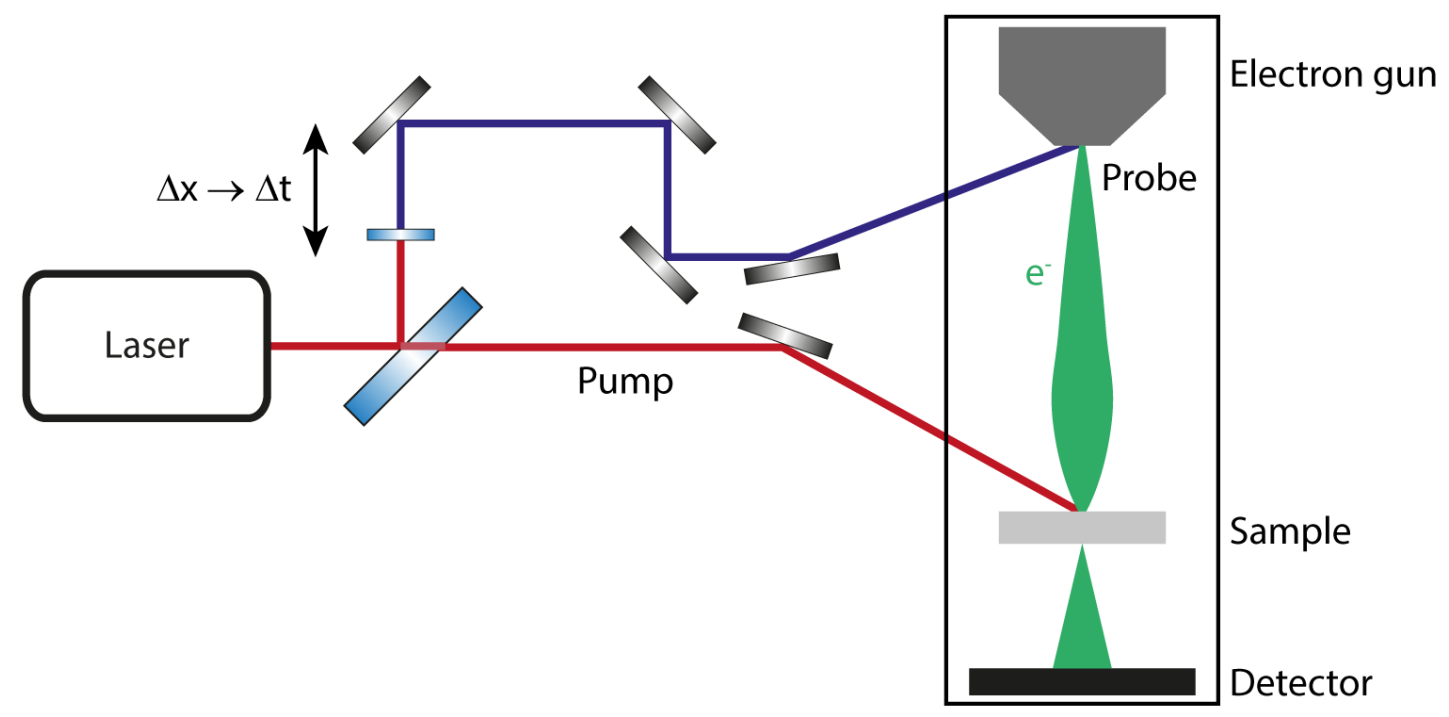

Figure 2.1: Pump-probe setup of an ultrafast electron microscope with a laser pump and electron probe beam.

be introduced into an existing electron microscope, e.g. a TEM [18, 24, 37]. Utilizing photoemission sources, the generation of ultra-short electron pulses is obtained via ultra-short laser pulses. The latter are typically generated using mode-locked laser systems [38], which are commercially available with a pulse duration of a few femtoseconds. Employing this approach, existing research methods can be used in order to extend investigations beyond mechanical or electronic shutter times into the pico- to femtosecond regime. For the implementation of such a photoemission gun, different concepts exists. An introduction into ultrafast electron guns is given in Sec. 2.3.

Due to the lack of detectors capable of directly measuring dynamics on the ultrafast timescale, a stroboscopic approach is used. In these so-called pump-probe experiments, the specimen is excited by a pump beam, leading to the desired change in the material, and subsequently probed by a second beam, measuring the response of the excited sample system. Varying the time-delay between the two pulses yields insight into the dynamics after excitation of the specimen. The temporal limit of this method is the pulse duration of the pump or probe beam, respectively. Hence, in order to resolve ultrafast dynamics, an ultrafast pump and probe beam is necessary. In case of a laser, as already mentioned, femtosecond pulses are not an issue. However, generating femtosecond electron pulses for an ultrafast electron microscope is challenging.

A pump-probe setup incorporating a TEM for electron diffraction or imaging is shown in Fig. 2.1. Here, the sample is pumped by a laser pulse and subsequently probed by 
an electron pulse. The temporal delay between pump and probe pulse is controlled by a variable path length of the laser pulse generating the electron probe pulse, which allows very precise control over the relative timing of the exciting and probing pulse. An additional path length in one of the arms of $\Delta x=0.3 \mu \mathrm{m}$ corresponds to a time delay of approximately $\Delta t \approx 1 \mathrm{fs}$.

Two general methods of achieving a temporal sequence using the pump-probe method can be distinguished: the single-shot and the repetitive approach. The single-shot approach is typically used to study irreversible processes, however, can also be used for reversible systems. In order to achieve a temporal sequence, not only the delay between pump and probe pulse, but also the investigated position on the specimen has to be changed (in case of irreversible processes). Hence, a homogeneous sample is required. For electron microscopy, the electron (probe) pulse in case of single-shot experiments has to contain at least $10^{6}-10^{7}$ electrons in order to achieve an image at the detector $[26,27]$, which leads to deteriorations of the pulse properties due to space charge effects.

If reversible processes are investigated, a repetitive approach can be used. Instead of obtaining an image with a single shot, the sample is pumped and probed multiple times. In case of a single-electron probe pulse, the experiment is required to be repeated $10^{6}-10^{7}$ times in order to receive an image on the detector. Thus, the integration time is increased compared to the single-shot experiment and depends on the number of necessary probe pulses and the repetition rate of the setup. The repetition rate on the other hand has to be low enough for the excitation of the sample system to fully decay. Compared to the single-shot approach, the repetitive method can be used to investigate inhomogeneous samples, since there is no requirement of changing the pump and probe pulse position.

\subsection{Conventional electron sources}

This section introduces the main electron sources used in electron microscopes. Generally, two different processes of emitting electrons are utilized in electrons guns: thermionic emission and field emission. The corresponding electron guns can be further distinguished in terms of the source optics, as thermionic gun assemblies are built to form a real crossover, whereas field emission electron guns only have a virtual crossover (see 
A

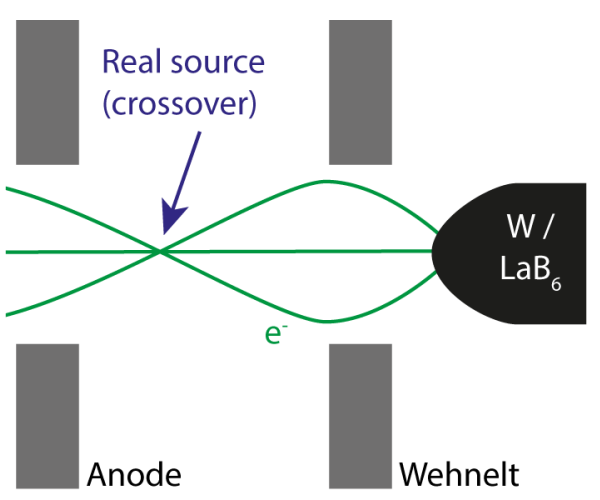

B

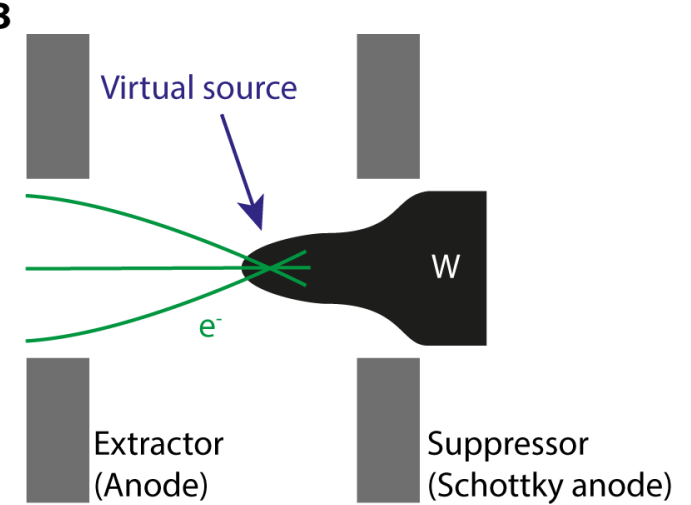

Figure 2.2: Schematic setup of an electron source. A: Real crossover design as used in thermionic electron guns. B: Gun design with a virtual source. Images adapted from Ref. [39].

Fig. 2.2). The term electron gun refers to the electron source and electrodes in the vicinity of the emitter. It is one of the most important parts of an electron microscope, and its design significantly affects the beam properties.

\subsubsection{Thermionic source}

In order to be emitted from a solid into vacuum, an electron has to overcome the work function $(\Phi)$ of the material. Electron emission from solid materials arising from high temperatures is referred to as thermionic emission. For sufficient temperatures, the Fermi-Dirac distribution is broad enough so that the high energy tail provides electrons with energies above the vacuum level, thus overcoming the work function. However, the required temperature is quite high, and for most metals on the order of several thousand Kelvin. With increasing temperature, the number of electrons which are able to escape rapidly increases. A term for the current density is given by the Richardson equation

$$
J_{R} \propto T^{2} \exp \left(-\frac{\Phi}{k_{B} T}\right)
$$

where $T, \Phi$ and $k_{B}$ denote the temperature, work function and Boltzmann constant, respecitvely. The constant of proportionality consists of the Richardson constant $A=$ $\frac{m_{e} e k_{B}^{2}}{2 \pi^{2} \hbar^{3}}$ ( $m_{e}$ : electron rest mass, $e$ : elementary charge and $\hbar$ : reduced Planck constant). Sometimes, it includes additional correction factors as well [40-43], or the Richardson constant itself is referred to as a material-dependent constant [44]. 

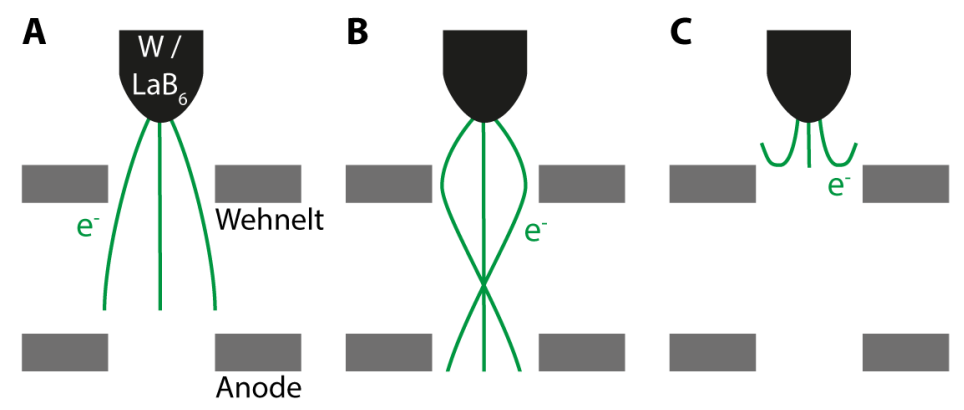

Figure 2.3: Electron emission for different bias voltages of the Wehnelt electrode. A: No bias, max. current B: Optimum bias, intermediate current c) High bias, no current. Images adapted from Ref. [39].

An electron gun utilizing thermionic emission is analogously called thermionic source. For a thermionic electron gun, temperatures corresponding to an energy of a few electronvolts are necessary to emit electrons. However, most materials will melt or even vaporize when exposed to such thermal conditions. To overcome this problem, either a material with high melting temperature (tungsten) or one with a low work function (Lanthanum hexaboride, $\mathrm{LaB}_{6}$ ) is needed.

A thermionic tungsten emitter consist of a tungsten filament that is bent in a V-shape ("hairpin"). The diameter of the tungsten wire is typically in the range of 100 to $300 \mu \mathrm{m}$ and the wire is heated by a direct current. Tungsten is an advantageous material for an electron source, as it has a high melting temperature, a low vapor pressure and is an electrical conductor. However, since it has to be heated to $2500-3000 \mathrm{~K}$, the lifetime of approximately 100 hours is rather low for such an emitter [45]. The alternative approach of using $\mathrm{LaB}_{6}$ has the advantage of a low work function, which reduces the necessary temperature significantly $(1400-2000 \mathrm{~K})$. As a result, the typical lifetime of $500-2000$ hours is higher $[39,45,46]$. Due to bad electrical conductivity, $\mathrm{LaB}_{6}$ has to be externally heated (e.g. by wires around the tip) [47, 48]. In addition, it is more susceptible to thermal shock. As mentioned before, thermionic sources are built into designs exhibiting a crossover electron gun. A Wehnelt electrode is assembled opposing the filament and, except of a small hole for the electrons to pass through, completely surrounds the emitter. The Wehnelt cylinder controls the emission current (cf. Fig. 2.3). Its potential is more negative than the emitter potential, reducing the emission area to the vicinity of the tip front and converging the electrons to the crossover point. Still, the effective source size (crossover size) is in the range of $10-40 \mu \mathrm{m}$ for these thermionic sources [39, 44, 45]. 
A

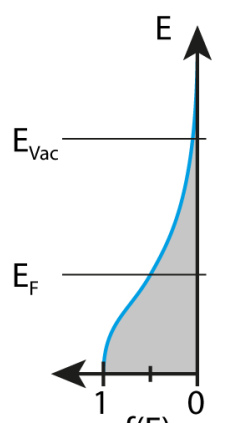

B

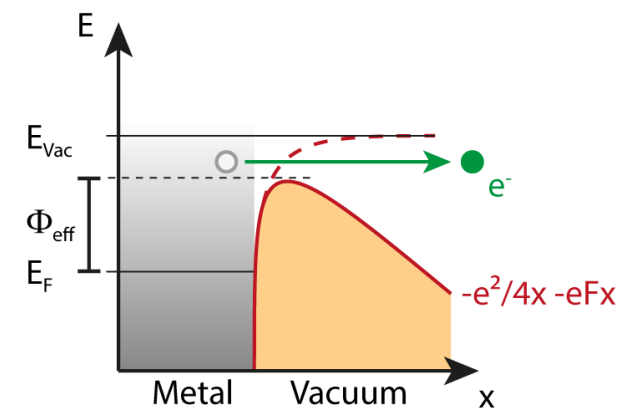

Figure 2.4: A: Exemplary Fermi-Dirac distribution with high-energy tail above the effective work function. B: Schematic depiction of the Schottky emission employing a hot electron distribution and an applied electric field $F$.

\subsubsection{Schottky emitter}

By introducing an electric field at the surface of a metal, e.g., by applying a voltage between the material and an electrode, the work function is reduced for proper electric field directions (see effective work function in Fig. 2.4). This so-called Schottky effect can be used in combination with thermionic emission to reduce the required temperature for operation.

The potential outside the metal becomes

$$
V(x)=\Phi-\frac{e^{2}}{16 \pi \varepsilon_{0} x}-\frac{e F x}{4 \pi \varepsilon_{0}},
$$

where $x$ is the coordinate pointing out of the metal normal to the surface, $\frac{-e^{2}}{16 \pi \varepsilon_{0} x}$ the contribution from the image force and $-\frac{e F x}{4 \pi \varepsilon_{0}}$ from the externally applied field $F$. The maximum of this potential curve and thus the effective work function is at $[39,49,50]$

$$
\Phi_{e f f}=\Phi-\sqrt{\frac{e^{3} F}{4 \pi \varepsilon_{0}}} .
$$

This field enhanced thermionic emission is called Schottky emission, and the current density is described by the Schottky equation [51]

$$
J_{S} \propto T^{2} \exp \left(\frac{e^{3 / 2} F^{1 / 2}}{\left(4 \pi \varepsilon_{0}\right)^{1 / 2} k_{B} T}-\frac{\Phi}{k_{B} T}\right)
$$

with the electric field $F$ and the vacuum permittivity $\varepsilon_{0}$. For very low electric fields, the equation reduces to the Richardson equation of the thermionic emission, as one would 
expect.

The Schottky equation holds under the assumption of insignificant contributions from tunneling current. According to J. Orloff (Ref. [39]), for electric fields, where less than approx. $30 \%$ of the emitted electrons are due to tunneling emission, Eq. 2.4 remains sufficient. Beyond that, the Schottky equation can be extended to remain adequate until about $70 \%$ tunneling contribution. The current density $J_{E S}$ in this extended Schottky regime is

$$
J_{E S}=J_{S} \frac{\pi q}{\sin \pi q}
$$

with $q=1.656 \cdot 10^{-4} \frac{F^{3 / 4}}{T}$ being a measure for the tunneling current contribution ${ }^{3}$ [39].

The implementation of Schottky emission into an electron gun can be seen as a trade-off between thermionic guns with their low lifetime and relatively poor beam characteristics and field emission sources (see next section), which require very good vacuum conditions.

In contrast to thermionic emitters, here, instead of a Wehnelt electrode in front, the tip is protruding through a suppressor electrode. Therefore, only a virtual source size exists, since there is no real crossover within the electron gun (cf. Fig. 2.2). The suppressor has, similar to the Wehnelt cylinder, a more negative potential than the tip and is responsible for preventing electron emission from the tip shank.

In Schottky emitters, a $\langle 100\rangle$ oriented tungsten tip is employed and additionally coated with a zirconium oxide $\left(\mathrm{ZrO}_{2}\right)$ layer to reduce the work function to about $2.6-2.95 \mathrm{eV}$ $[39,45,52]$. The lifetime greatly benefits from the lower work function, since it reduces the necessary temperature and applied electric field. In order to achieve a $\mathrm{ZrO}_{2}$-coating, zirconium hydride $\left(\mathrm{ZrH}_{2}\right)$ is placed onto the shank distant from the tip apex in the form of a droplet [53-55]. By heating the tip, the $\mathrm{ZrH}_{2}$ decomposes into zirconium and hydrogen. The hydrogen evaporates, leaving a $\mathrm{Zr}$ droplet. In a next step, the emitter is heated in a partial pressure of oxygen (so-called oxygen treatment), allowing for the formation of a $\mathrm{Zr}-\mathrm{O}$ complex [53, 54, 56-58]. This $\mathrm{Zr}-\mathrm{O}$ complex diffuses several hundred tungsten layers into the bulk, as well as to the front facet of the tip by both, surface and bulk diffusion [57, 58]. Furthermore, the zirconium of the droplet is transformed into zirconium (di-)oxide, leaving a reservoir of zirconium and oxygen [53, 54]. Hence, the emitter only lasts as long as the reservoir is not depleted. Additional

\footnotetext{
${ }^{3}$ A value of $q=0.3$ means that approximately $30 \%$ of the overall current is due to tunneling.
} 
oxygen treatments are not necessary, since the reservoir is now consisting of $\mathrm{ZrO}_{2}$. Upon

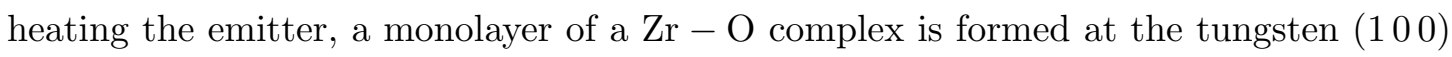
surface, reducing the work function to about $2.7 \mathrm{eV}$ [57-60]. The lowering of the work function is highly selective to the tungsten orientation and occurs solely at the W( $\left.\begin{array}{lll}1 & 0 & 0\end{array}\right)$ surface, leading to a high confinement of the electron beam along the $\langle 100\rangle$ direction $[56,57]$. For, e.g., the (111)-surface, the work function is only slightly lowered to about $4.8 \mathrm{eV}[61]$.

\subsubsection{Field emission gun}

As already known from Schottky emission, if an electrostatic field is applied at a solid surface, the potential barrier is bent as a result. Therefore, for sufficiently high fields, the width of the potential barrier can become small enough for quantum mechanical tunneling of electrons through the barrier. This process is called field emission or FowlerNordheim tunnel emission.

The corresponding current density is described by the Fowler-Nordheim equation [43, $62]$ :

$$
J_{F N} \propto F^{2} \exp \left(-\frac{4 \sqrt{2 m_{e}}}{3 e \hbar} \frac{\Phi^{3 / 2}}{F}\right) .
$$

For this equation, the constant of proportionality is complex, and the reader is referred to Ref. [62-66] for more detailed information. Typical values of the required electric field are on the order of $10^{9}-10^{10} \frac{\mathrm{V}}{\mathrm{m}}$ for field emission from metals [67].

The setup of a field emission gun is rather simple, as it comprises only the emitter and an opposing anode. Since the tip is not heated, there is no need for a Wehnelt or Schottky electrode. Utilizing field emission to extract electrons has the advantage of achieving even better beam properties than a Schottky emitter, since the emission is more localized.

As mentioned above, a field strength of $10^{9} \frac{\mathrm{V}}{\mathrm{m}}$ is necessary for a significant tunneling current. Instead of applying a corresponding voltage to a flat cathode, the way to go is to apply a relatively moderate voltage to a very sharp tip utilizing the so-called lightning rod effect. By approximating the tip as a sphere with radius $r$, the local electric field can be calculated by $F=U / r$ for an applied potential $U$. This formula is extended by an empirical geometry factor $k$ to match the field of an actual tip which is reduced due 
to the presence of the shank:

$$
F=\frac{U}{k r}
$$

The value of $k$ is typically in the range of $4-6$ for most emitter geometries [43, 67, 68].

The preferred material for field emitters is again tungsten, with the advantages stated in the previous section. Furthermore, it can be etched easily from a thin wire to a tip with an apex radius on the order of about $100 \mathrm{~nm}$. This leads to a significant local enhancement of the electric field, and applying a potential on the order of $1-5 \mathrm{kV}$ already results in a tunneling current. The field emission depends on the work function and therefore on the crystallographic orientation of the tip. For tungsten, the best orientation would be $\langle 310\rangle$, with a work function of about $4.2-4.35 \mathrm{eV}[44,69,70]$. However, also $\langle 111\rangle$ oriented tungsten is used, as it is more stable with regard to mechanical forces $[71,72]$. To sustain the work function, the surface has to be free of contamination. Therefore, the emitter has to be operated under ultra-high vacuum conditions, i.e., at a pressure of $10^{-10}$ mbar or below $[44,45]$. Even under those conditions, the emitter needs to be heated ("flashed") from time to time to remove adsorbate layers.

\subsubsection{Summary}

In summary, field emission guns are best in terms of their beam characteristics. The small source size leads to a very large spatial coherence and high current density. The drawbacks are the necessary ultra-high vacuum condition and, compared to Schottky emitters, the emission current is not as stable. A Schottky emitter is a good trade-off between beam properties and reliable, easy operation. Furthermore, since the surface contamination does not occur in the same way as for the field emission gun, the necessary vacuum pressure can be $1-2$ orders of magnitude higher. Thermionic guns only present advantages when operating at relatively low magnification $(\ll 100.000 \times)$, since the small source size of a field emitter leads to a loss in current density when illuminating large areas [46]. For comparison, different parameters of the introduced electron guns are shown in Tab. 2.1. 


\begin{tabular}{|c|c|c|c|c|}
\hline Electron source & $\begin{array}{l}\text { Thermionic } \\
(\mathrm{W})\end{array}$ & $\begin{array}{l}\text { Thermionic } \\
\left(\mathrm{LaB}_{6}\right)\end{array}$ & $\begin{array}{l}\text { Schottky } \\
\text { emitter }\end{array}$ & $\begin{array}{l}\text { Field } \\
\text { emitter }\end{array}$ \\
\hline Material & W & $\mathrm{LaB}_{6}$ & $\mathrm{ZrO} / \mathrm{W}$ & $\mathrm{W}$ \\
\hline Work function $(\mathrm{eV})$ & 4.5 & 2.7 & 2.7 & 4.5 \\
\hline Operation temp. (K) & $2500-3000$ & $1400-2000$ & 1800 & 300 \\
\hline Tip radius $(\mu \mathrm{m})$ & 60 & 10 & $0.4-1$ & $<0.1$ \\
\hline Electric field $\left(\mathrm{V} \mathrm{m}^{-1}\right)$ & Low & Low & $10^{8}$ & $>10^{9}$ \\
\hline Current density $\left(\mathrm{A} \mathrm{m}^{-2}\right)$ & $10^{4}$ & $10^{5}-10^{6}$ & $10^{7}-10^{8}$ & $10^{8}-10^{10}$ \\
\hline Total emission current $(\mu \mathrm{A})$ & 200 & 80 & $100-500$ & $5-10$ \\
\hline Brightness $\left(\mathrm{A} \mathrm{m}^{-2} \mathrm{sr}^{-1}\right)$ & $10^{9}$ & $10^{10}$ & $10^{11}$ & $10^{12}-10^{13}$ \\
\hline Source size & $15-50 \mu \mathrm{m}$ & $5-20 \mu \mathrm{m}$ & $15-20 \mathrm{~nm}$ & $2.5-10 \mathrm{~nm}$ \\
\hline Vacuum pressure (mbar) & $<10^{-5}$ & $<10^{-6}$ & $<10^{-9}$ & $<10^{-10}$ \\
\hline Lifetime $(\mathrm{h})$ & 100 & 1000 & $>5000$ & $>2000$ \\
\hline Energy spread (eV) & $1.5-3$ & $1-2$ & $0.3-0.7$ & 0.3 \\
\hline
\end{tabular}

TABLE 2.1: Operation parameters for different electron sources. Values combined from Refs. $[45,73,74]$.

\subsection{Pulsed electron sources}

This section starts with an introduction to photoemission from metals, and shortly discusses the nonlinear emission mechanisms and their (dis-)advantages. Thereafter, different gun designs in order to generate ultrafast electron pulses are presented.

\subsubsection{Photoemission from metal surfaces}

The photoelectric effect describes the excitation and subsequent emission of an electron after the absorption of a single photon with an energy $\hbar \omega$ (Fig. 2.5A). The photon energy is required to be larger than the work function of the material. The maximum excess energy is hence the difference in photon energy and work function as stated by Einstein's photoelectric law $[75,76]$

$$
E_{k i n}=\hbar \omega-\Phi .
$$

The number of emitted electrons follows a linear dependency on the incident light intensity. For photons with insufficient energy, i.e., $\hbar \omega<\Phi$, single-photon electron emission cannot occur. This restriction, however, can be relaxed for high intensities in the sense that the required energy can stem from more than one photon. In 1931, Göppert-Mayer presented a perturbation theory calculation for two-photon absorption [77]. However, the necessary light intensities could not be reached at the time. Only with the advent of 
A

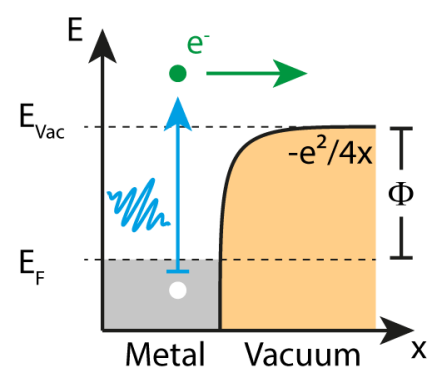

B

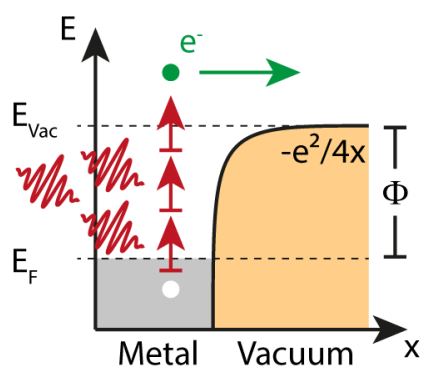

C

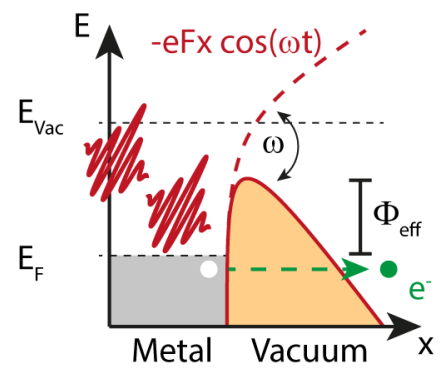

Figure 2.5: Sketches of photoemission at a metal surface. $E_{F}$ denoting the Fermilevel and $E_{v a c}$ the vacuum level. A: Normal (single-photon) photoelectric effect, where an electron is excited above vacuum level by a single photon with an energy larger than the work function $(\hbar \omega>\Phi)$. B: Multi-photon emission: Several photons with energy $\hbar \omega>\Phi$ are simultaneously absorbed in order to excite one electron. C: Optical field emission regime (Keldysh parameter below one) achieved upon strong laser excitation.

laser systems, the required intensities for this improbable process became achievable. In 1961, Franken et al. could demonstrate the effect of second harmonic generation, where a suitable material emits a photon with twice the energy of the incident photon energy [78]. This experiment demonstrated the importance of laser light for nonlinear optics. For photoemission, this means that it is possible to generate electrons by simultaneously absorbing two or more photons with energy below the work function (Fig. 2.5B). The cross-section for nonlinear photoemission decreases for increasing nonlinearity, i.e., for increasing number of necessary photons. The current density scales with the power of the number of photons absorbed in intensity [79-81]:

$$
J_{N P} \propto I^{n},
$$

where $n$ is the number of absorbed photons. This so-called multiphoton photoemission has been studied intensively in gases $([82-86])$ as well as solid materials [79-81, 8789]. Latter especially in case of metal nanotips [28-32, 90-92]. Applying a static bias voltages can lead to a decrease in the nonlinearity of the emission process [31, 93]. For increasing intensities, the absorption of more photons than the minimum number required for photoemission (or ionization) might occur [82, 84, 94-96]. This process is called above-threshold photoemission (or above-threshold ionization).

By increasing the light intensity even further, new processes become dominant: the so-called optical field emission or strong field ionization. Here, "strong" refers to the optical field strength becoming larger than the binding fields [29, 92, 97, 98]. In case of 
solid materials, the optical fields are strong enough to significantly bend the potential barrier at the material surface. This allows for electrons to be able to tunnel through the potential barrier within one half cycle of the laser pulse, in a process similar to static field emission (Fig. 2.5C) [29, 92, 99, 100].

The transition between strong and weak fields is characterized by the Keldysh parameter $\gamma$, defined as

$$
\gamma=\sqrt{\frac{\Phi}{2 U_{p}}},
$$

where $U_{p}$ is the ponderomotive potential [101]. The ponderomotive potential is a quantity for the mean kinetic energy of an electron within an oscillating field, here the laser electric field, and can be calculated using

$$
U_{p}=\frac{e^{2} F^{2}}{4 m \omega^{2}}
$$

Here, $F$ denotes the laser electric field, $\omega$ the frequency of the laser, $m$ the electron mass and $e$ the elementary charge.

\section{Nonlinear photoemission from metal nanostructures}

Nonlinear photoemission in combination with a non-uniform electric field offers the possibility to emit electrons with a high spatial localization $[28,31,33]$. This is particularly interesting for ultrafast electron microscopy, where ultra-short electron pulses with a low emittance are desired.

The non-uniform electric field is crucial to realize localization beyond the laser focus size. To this end, metal nanotips with a radius of curvature on the order of a few tens of nanometers at the apex are utilized. Due to the geometry of the metal conductor, electric field lines are concentrated at the sharpest point, also known as the lightning rod effect. The enhancement of the local electric field at visible to near-infrared wavelengths is typically in the range of $3-12$ for a tungsten tip with an apex diameter of about $20-40 \mathrm{~nm}$ [32, 102-105]. For gold tips, the enhancement can be even larger due to resonant excitation of surface plasmons $[102,106]$.

The static electric field distribution can be calculated analytically in case of paraboloidal or hyperboloidal tip-anode geometries [107]. A paraboloidal solution of the electric field 


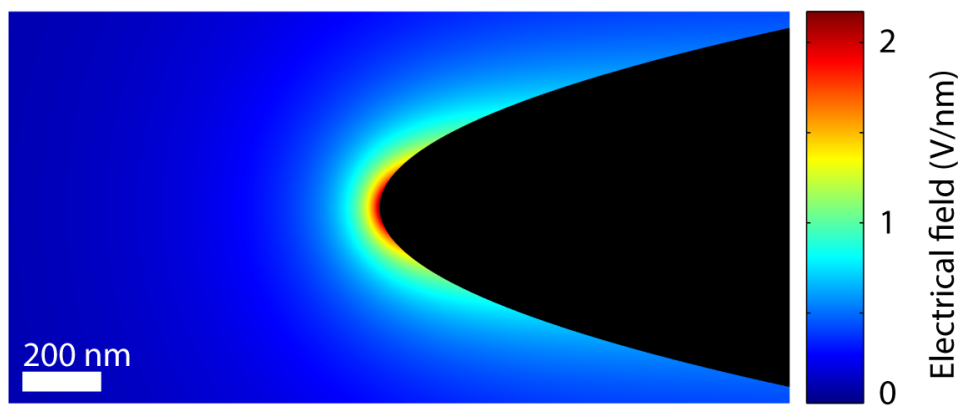

Figure 2.6: Electrostatic field distribution calculated based on Ref. [108] for a paraboloidal tip-anode geometry with $100 \mathrm{~nm}$ apex radius and a potential of $1000 \mathrm{~V}$ applied between the tip and an anode at a distance of $1 \mathrm{~mm}$.

is shown in Fig. 2.6 for a tip with $100 \mathrm{~nm}$ apex radius and a potential of $1000 \mathrm{~V}$, which is applied between the tip and an anode at a distance of $1 \mathrm{~mm}$.

As introduced in Sec. 2.3.1, depending on the illumination conditions, different regimes of electron emission can be reached. In case of multiphoton photoemission and considering the field enhancement at the apex, it is evident that the higher the nonlinearity, the better the confinement of the electron emission to the tip apex. For an n-photon photoemission process, the ratio between the number of electrons emitted from the apex and shaft region can be estimated to be

$$
\frac{N_{\text {apex }}}{N_{\text {shaft }}}=\frac{A_{\text {apex }} \beta^{2 n} I^{n}}{A_{\text {shaft }} I^{n}}=\frac{A_{\text {apex }} \beta^{2 n}}{A_{\text {shaft }}} .
$$

Assuming a half-sphere apex surface with an apex radius of $100 \mathrm{~nm}$, a laser focus size of $15 \mu \mathrm{m}$ and an opening angle of the tip emitter of $10^{\circ}$ (cf. Fig. 4.2B), the ratio of the emission areas is approximately

$$
\frac{A_{\text {apex }}}{A_{\text {shaft }}}=\frac{2 \pi(100 \mathrm{~nm})^{2}}{\pi(15 \mu \mathrm{m})^{2} \cdot 10^{\circ} / 360^{\circ}}=0.0032 .
$$

Further assuming a field enhancement at the apex of $\beta \approx 5$, Tab. 2.2 shows values for the ratio of apex to shaft yield obtained for one- to four-photon photoemission processes. Already for a nonlinear process where two or more photons are necessary, electron emission from the apex region is dominant. The ratio of approximately $2: 1$ for two-photon photoemission is sufficient in the TEM-emitter geometry, since the emission site can be selected (see Chap. 5).

Upon further increase of the incident laser intensity on such nanostructures, a transition 


\begin{tabular}{cc} 
Number of photons $n$ & $N_{\text {apex }} / N_{\text {shaft }}$ \\
\hline 1 & 0.08 \\
2 & 2 \\
3 & 50 \\
4 & 1250 \\
\hline
\end{tabular}

TABLE 2.2: Ratio of emitted electrons stemming from the apex and shaft region for different single- and multi-photon photoemission processes.
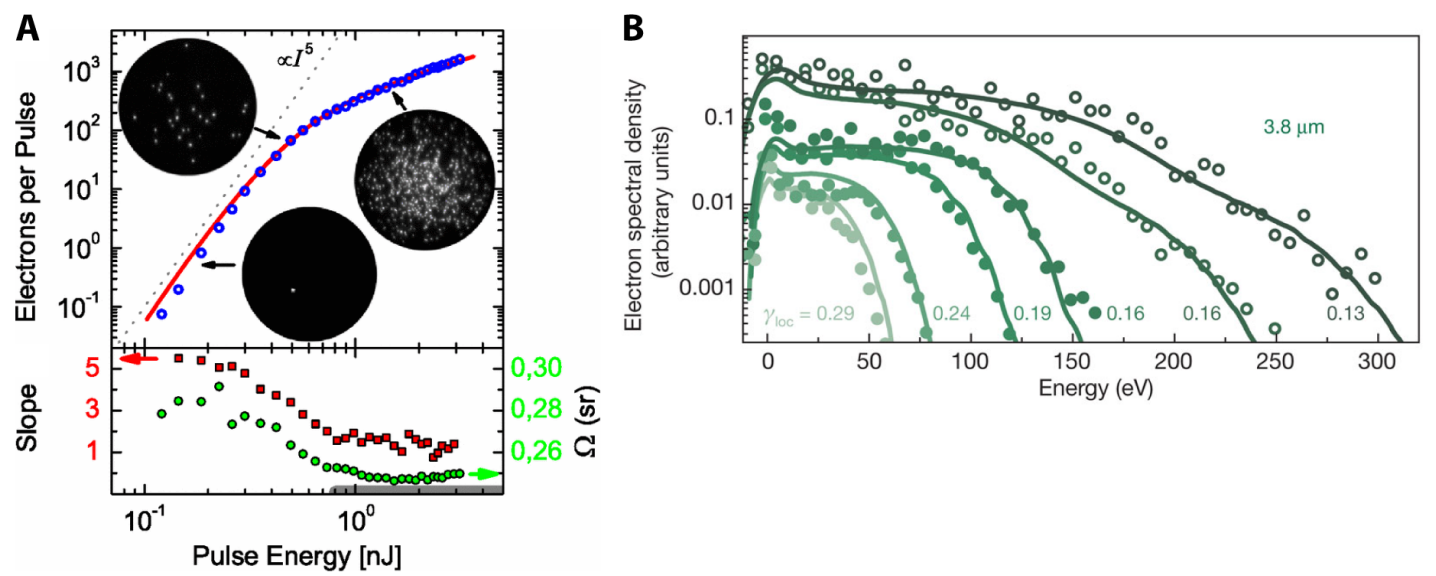

Figure 2.7: A: Electron yield indicating the transition from the multi-photon to the strong-field regime, measured with a gold nanotip at $800 \mathrm{~nm}$ wavelength. Figure from Ref. [29]. B: Kinetic energy distribution of photoelectrons for different intensities at a constant wavelength of $3.8 \mathrm{um}$ in the strong-field regime. Reprinted by permission from Macmillan Publishers Ltd: Nature (Ref. [92]), copyright (2012).

into the strong-field regime occurs $[29,31,91,109]$. The required field strength is in the range of $10^{13} \frac{\mathrm{W}}{\mathrm{cm}^{2}}$ for, e.g., $800 \mathrm{~nm}$ laser light ${ }^{4}$. Such intensities can be acquired utilizing the field enhancement at the apex of a sharp metal tip in combination with a femtosecond laser pulse. On the other hand, these intensities are also in the range of the damage threshold $[110,111]$. A hallmark of the strong-field regime is a strong interaction between the laser field and the kinetic energy of emitted electrons [92, 97, 112]. The transition from the multiphoton to the strong-field regime occurs at Keldysh parameters around unity and is identified by a decrease of the intensity dependent electron yield (see Fig. 2.7A) [29]. The transition occurs around $\gamma \approx 1$ due to rapid closing of lowerorder emission channels and is accompanied by an increase in forward momentum gained from the laser field, thus reducing the solid angle of emission $\Omega$. To reach further into the strong-field regime, in order to prevent material damage, the wavelength has to be increased (cf. Eq. 2.10). Upon decreasing the Keldysh parameter, a second feature of the strong-field regime can be observed: The broadening of the electrons kinetic.

\footnotetext{
${ }^{4}$ In order to reach a Keldysh parameter of one using Eq. 2.10, Eq. 2.11 and a work function of $4.5 \mathrm{eV}$.
} 
A

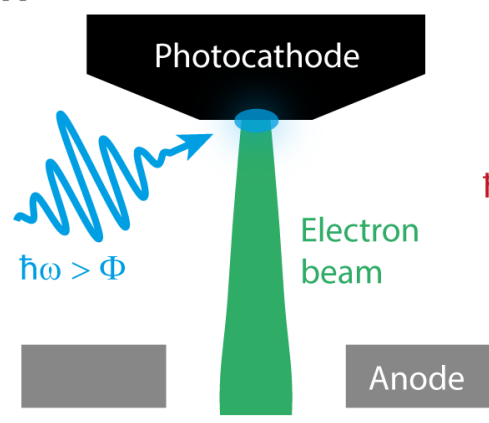

B

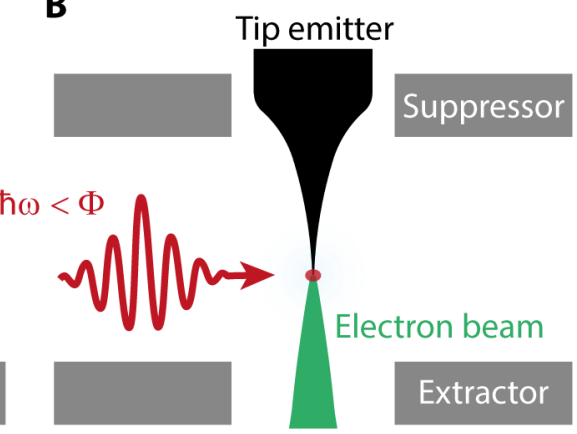

FiguRE 2.8: Schematic of the two main approaches in ultrafast electron gun design. A: A flat photocathode illuminated by an UV-laser pulse (photon energy larger than the work function of the photocathode $\Phi$ ). B: Tip based approach, where visible to near-IR-laser pulses in combination with nonlinear photoemission generate localized electron pulses.

This broadening is a result of the quiver motion in the driving (laser) field an electron is undergoing while escaping the near-field of a sharp metal tip, and scales with the ponderomotive potential [92]. Such energy spectra are shown in Fig. 2.7B for a gold-tip illuminated at mid-infrared frequencies [92].

Due to the large energy spread, the strong-field regime is not suited for ultrafast electron microscopy, as the kinetic energy spread should be kept as small as possible to maintain coherence and reduce aberrations.

\subsubsection{Gun design for pulsed electron sources}

The common design used for an ultrafast electron gun consists of a flat photocathode with an opposing anode (see Fig. 2.8A) [7, 12, 113-115]. Recently, three alternative approaches were investigated. The first one utilizes a sharp metal tip in a Schottky emitter setup (see Fig. 2.8B) [17, 35, 116]. The second approach of generating ultra-short electron pulses is by photoionizing an ultra-cold gas [117-119]. A third method generates electrons using a flat photocathode as well, however, incorporating a radio frequency compressor in order to achieve short electron pulses [26, 120-123]. All three designs come with respective advantages and disadvantages. The common flat photocathode and the tip based source will be shortly introduced.

\section{Flat photocathode}

Ultrafast electron diffraction and imaging experiments are nowadays mostly based on 
flat photocathodes illuminated by ultra-short laser pulses (cf. Fig. 2.8A) [7, 12, 113-115]. In these setups, an ultraviolet laser pulse generates electrons from either a metal film or a solid metal cathode. The emission process is usually single-photon photoemission, as the photon energy of the laser light $(\hbar \omega)$ is larger than the work function $(\Phi)$ of the photocathode material. The generated electrons are subsequently accelerated towards an anode opposing the emitter (cf. Fig. 2.8A).

This design can be used in the single-electron regime, where only one electron per laser pulse is generated, or to perform single-shot experiments. In order to obtain an image with one shot, at least $10^{6}-10^{7}$ electrons are necessary $[26,27]$. The single-shot mode allows to investigate non-reversible dynamics [6, 124], whereas the single-electron regime is limited to reversible processes.

On the downside, a flat photocathode suffers from a large source size, since the source size in this design is mainly determined by the laser focus size. Furthermore, if not driven in single-electron mode, Coulomb interaction may become dominant. The space charge effect is the most prominent effect and generally describes the deflection due to electron-electron interactions [39]. Space-charge effects are typically limiting the pulse duration to the picosecond regime, or, at best, a few hundred femtoseconds [26, 125]. Such electron-electron interactions occur especially within a focus or crossover of the electron beam and lead to deterioration of the beam parameters which can also affect the brightness [39, 126].

\section{Tip based source}

In this work, we investigate an alternative design based on the classical Schottky or field emission gun, employing a sharp tungsten needle emitter. A schematic illustration of the design is depicted in Fig. 2.8B. In this tip based approach, the idea is to generate electrons only at the very apex of the tip. Again, an anode (extractor) accelerates the electrons. Furthermore, a suppressor electrode restricts electrons emitted from the tip shaft to be transmitted through the anode aperture. The suppressor electrode is built into the (conventional) Schottky emitter to prevent thermionic emission from the tip shank and the wire connected to it. However, in case of a photoemission source, the suppressor is helpful to control the emission site of photoemitted electrons as well, which will be discussed in the following chapters of this thesis. The control over the emission site is especially important for low-order nonlinear or single-photon photoemission, where 
electron emission from the tip apex is not dominating the emission from the shaft (see below).

The main advantage of the tip based approach is, compared to the flat photocathode, the confinement of the emission site. An exemplary apex diameter of $100 \mathrm{~nm}$ in case of the tip-based approach compared to a typical focus size of about $10 \mu \mathrm{m}$ for a flat photocathode yields a much larger confinement of the emission size. Thus, intrinsically a much smaller emittance.

\subsection{Electron beam characteristics}

To be able to compare different electron microscopes, electron sources and, as an indication of the capabilities of a setup, e.g. the minimal focus size, the properties and characteristics of an electron beam are important. Even though they can be manipulated by lenses and apertures, the emittance, brightness and coherence are usually parameters initially governed by the electron gun.

\subsubsection{Emittance}

An electron beam has a certain spread in kinetic energy. This can, for example, stem from thermal velocity spread of electrons, or emission from different energy levels. Furthermore, electrons are emitted with different angles with respect to the emitter surface. To be able to describe the beam quality, a parameter taking into account directions and velocities or the width and divergence of the electron beam is necessary. This quantity is called emittance. Unfortunately, the definition of emittance is not standardized and not used consistently throughout literature. The definition employed here is based on the concept of Reiser [127]. To neglect space charge effects and forces, such as the Boersch effect, the beam is considered to have a sufficiently low current density and hence can be treated as a set of independent rays.

Electrons are defined by the space coordinates $x, y, z$ and momentum coordinates $p_{x}$, $p_{y}, p_{z}$. Throughout this thesis, the electron beam propagates along the $z$-direction. 
The slope of an electron trajectory is given by

$$
x^{\prime}=\frac{d x}{d z}=\frac{d x d t}{d t d z}=\frac{\dot{x}}{\dot{z}}=\frac{p_{x}}{p_{z}}
$$

within the $x-z$ plane. For a fixed $z$-coordinate, every electron then incorporates a point in the $x-x^{\prime}$ space, the so-called "trace space". The area obtained by integrating over the trace space is

$$
A_{x}=\iint d x d x^{\prime}
$$

which is often referred to as a geometric definition of the emittance [127, 128]. Correspondingly, the same calculation applies for the $y-y^{\prime}$ trace space. The two emittances in $x$ - and $y$-direction $\left(\varepsilon_{x}, \varepsilon_{y}\right)$ are referred to as transverse emittance and taken into account separately. However, if the electron beam is axially symmetric, a radial emittance $\varepsilon_{r}$ is sufficient.

The emittance defined in the above stated manner is inadequate though, since it depends on the kinetic energy of the electrons. This means that for increasing $p_{z}$, that is for increasing accelerating voltage, the slope $x^{\prime}$ decreases as $p_{x}$ stays the same. Therefore, a normalization of the emittance is necessary to compare beams with different energies. With the relativistic energy and velocity factors $\gamma=1 / \sqrt{1-\beta^{2}}$ and $\beta=v_{e} / c, c$ being the speed of light and $v_{e}$ the electron velocity, the normalized emittance reads

$$
\varepsilon_{n, x}=\beta \gamma \varepsilon_{x}
$$

Alternatively, the emittance is defined in terms of beam width and divergence by central moments of the particle distribution $[49,127,129]$ :

$$
\varepsilon_{x}=\sqrt{\left\langle x^{2}\right\rangle\left\langle x^{\prime 2}\right\rangle-\left\langle x x^{\prime}\right\rangle^{2}}
$$

This version is called root mean square emittance (RMS emittance). The brackets $\langle\cdot\rangle$ indicate an average value. Hence, the RMS emittance is a quantity of the electron beam width $\left(\left\langle x^{2}\right\rangle\right)$, the velocity spread $\left(\left\langle x^{\prime 2}\right\rangle\right)$ and the term $\left(\left\langle x x^{\prime}\right\rangle^{2}\right)$.

It is usually reasonable to assume that the forward momentum is much larger than the transverse momentum $\left(p_{x} \ll p_{z}\right)$. Hence, in Eq. 2.14, the average longitudinal momentum of an electron bunch, expressed as $\beta \gamma m_{e} c$, can be used in place of $p_{z}$. This 
results in an equation for the normalized emittance that reads

$$
\varepsilon_{n, x}=\frac{1}{m_{e} c} \sqrt{\left\langle x^{2}\right\rangle\left\langle p_{x}^{2}\right\rangle-\left\langle x p_{x}\right\rangle^{2}}
$$

In the waist of an ideal uniform beam, the covariance term $\left\langle x x^{\prime}\right\rangle^{2}$ vanishes, and the emittance can be written with the standard deviation $\sigma_{x}=\sqrt{\left\langle x^{2}\right\rangle}$ and $\sigma_{x^{\prime}}=\sqrt{\left\langle x^{\prime 2}\right\rangle}$ as

$$
\varepsilon_{x}=\sigma_{x} \sigma_{x^{\prime}}
$$

The term $\sigma_{x}$ can be considered as the minimal cross section of the electron beam and hence be calculated using $\sigma_{x}=\varepsilon_{x} \sigma_{x^{\prime}}$. The emittance is therefore a measure for the achievable spot size when the electron beam is focused using a certain angle $\alpha$. The angle $\alpha$, defined as

$$
\alpha \approx d x / d z=x^{\prime}
$$

is the angle between the optical axis and the electron trajectory. Here, used as the maximum angle. The emittance can then be rewritten in terms of the angular standard deviation: $\varepsilon_{x}=\sigma_{x} \sigma_{\alpha}$, or, in case of the normalized emittance:

$$
\varepsilon_{n, x}=\beta \gamma \sigma_{x} \sigma_{\alpha}
$$

\subsubsection{Brightness}

For an estimation of the electron beam quality, the emittance alone is insufficient. In principle, the emittance can be arbitrarily decreased by inserting smaller apertures, since no information about the actual number of electrons is included. However, in order to obtain an image within reasonable integration times, a statement about the electron current is necessary. The figure of merit is the current density in phase space, called brightness. There are different definitions of brightness in use throughout literature. Usually, the brightness is defined by the current density per solid angle [39, 46, 127, 128]:

$$
B=\frac{J}{d \Omega}=\frac{d I}{d A d \Omega}
$$

To calculate the average brightness, the total beam current confined in a given tracespace volume and the emittance is used. Latter is assumed to form a (hyper-) ellipsoid and therefore is proportional to the trace space area. The resulting definition reads 
[127]:

$$
\bar{B}=\frac{I}{8 \pi^{2} \varepsilon_{x} \varepsilon_{y}}
$$

A normalized brightness is given by

$$
B_{n}=B /(\beta \gamma)^{2}
$$

in analogy to the normalized emittance.

In addition to the normalized brightness introduced, a different normalization can be found in the literature. Usually referred to as reduced brightness, it is sometimes called normalized brightness as well. The definition is

$$
B_{r}=B / U
$$

with $U$ being the acceleration voltage. For small velocities, i.e. $v_{z} \ll c$, an estimation of the difference between the two definitions can be made. By approximating $v_{z} \approx v_{e}$, the product $\beta \gamma$ can be simplified to $\beta \gamma \approx v_{e} / c$. In terms of the acceleration voltage $U$, the square of the relativistic parameters can be written as $(\beta \gamma)^{2} \approx 2 e U /\left(m_{e} c^{2}\right) \approx 4 \cdot 10^{-6} U$. Hence, the normalized brightness is larger than the reduced brightness by a factor of $2.5 \cdot 10^{5}$.

In this thesis, the definition of the normalized average brightness $\left(\bar{B}_{n}\right)$ is used, and will be referred to as "brightness". The brightness remains constant throughout the beam path, as long as aberrations can be neglected [71].

\subsubsection{Coherence}

The coherence of an electron wave denotes the ability to produce interference effects and depends on the phase correlation within an electron bunch. In Ref. [130] a more general definition is given, where coherence is defined by the correlation between quantities of an optical field. In electron microscopy, a coherent electron beam is necessary in order to good obtain image quality, e.g., sharp diffraction patterns or phase contrast images. Two different kinds of coherence can be determined - temporal (longitudinal) and spatial (transverse) coherence [130]. 
A

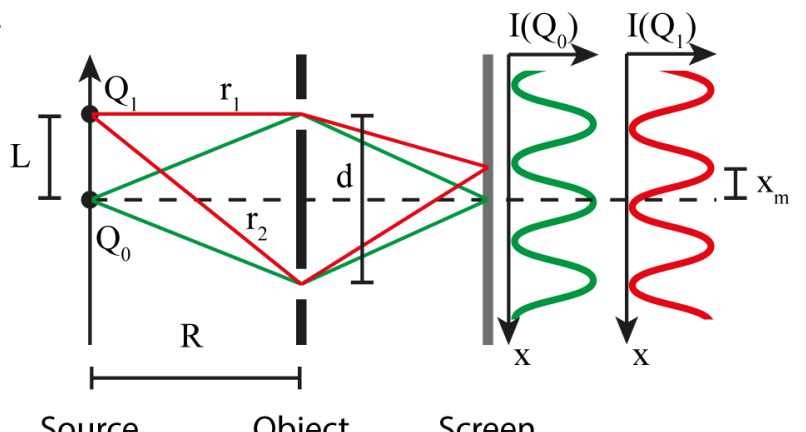

B

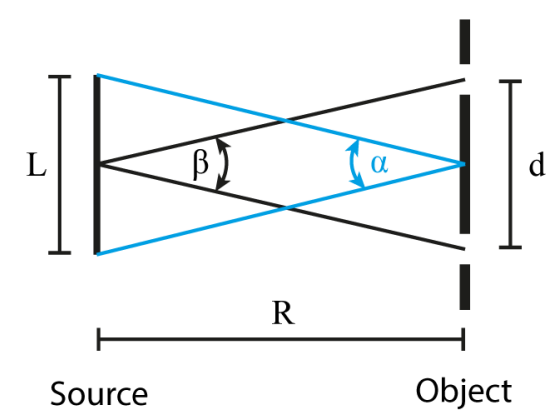

FIGURE 2.9: A: Sketch of Young's interference experiment with two beam paths for two point sources $Q_{0}$ (green) and $Q_{1}$ (red). B: Angles for the spatial coherence condition. Images adapted from Ref. [130].

Temporal coherence is a measure of how well a wave can interfere with itself in time and in propagation direction. In case of electron pulses, it therefore depends on the speed (or wavelength) of the electron bunch and the energy spread $\Delta E$ within an electron wave packet. The longitudinal coherence length is defined with the electron velocity $v_{e}$ and the Planck constant $h$ as

$$
d_{c, l o}=\frac{v_{e} h}{\Delta E}
$$

Considering an acceleration voltage of $U=120 \mathrm{kV}$ and an energy spread of $\Delta E=2 \mathrm{eV}$, the longitudinal coherence length calculates to $d_{c, l o} \approx 360 \mathrm{~nm}$. Compared to the transverse coherence, this value is significantly larger. Thus, the temporal coherence of the beam incident on the sample rarely limits the image quality in an electron microscope. However, for electrons transmitted through a specimen, due to energy losses, temporal coherence might affect the image quality. For compensation, energy-filters can be applied (see, e.g., Ref. [46] for more information).

The spatial or transverse coherence describes the capability to achieve interference patterns from scatterers that are far apart from each other. In case of electron sources, it reflects the source size. Therefore, perfect spatial coherence would correspond to electrons originating from a point source. In order to resolve complex systems, as for example cell structures in biology, a large spatial coherence length is necessary, since electrons scattered across the sample region have to interfere. With relevant molecules or structures on the order of nanometers this determines the order of the necessary coherence length [27].

For imperfect sources, spatial coherence can be nicely explained with a Young's interference experiment as illustrated in Fig. 2.9. An extended electron source can be treated 
as a composition of independent (single) point sources. For simplicity, two point sources $\left(Q_{0}, Q_{1}\right)$ are considered, separated by a distance $L$. A double-slit is introduced as the scattering object with the distance $\mathrm{d}$ between the holes and at a distance $R$ from the source.

By placing the first source on the optical axis, the phase of the wave at the two holes is the same. Subsequently the interference pattern has an intensity maximum on the optical axis $\left(I\left(Q_{0}\right)\right.$, see Fig. 2.9A). For the off-axis point source $Q_{1}$ the path length from the source to the slits are not equal and therefore a phase difference between the two waves exists. This results in a shift $x_{m}$ of the intensity pattern $I\left(Q_{1}\right)$ with respect to the on-axis pattern $I\left(Q_{0}\right)$ [130]. The path length difference can be expressed in terms of the geometrical setup by

$$
\left|r_{1}-r_{2}\right|=\frac{d L}{R}
$$

Upon simultaneous emission from $Q_{0}$ and $Q_{1}$ with a fixed phase difference between the two again only a shifted intensity pattern will be observed. However, for an incoherent source, where incoherent means that there is no fixed phase relation between $Q_{0}$ and $Q_{1}$, only the sum of the intensities from the two superimposed wave fields is observed. Hence, to be able to detect the fringes, the shift between the patterns of the point sources should remain low. Especially, the minimum of one pattern must not overlap with the maximum of the other. This case occurrences for the first time at a path difference of half the (electron) wavelength. Therefore the following condition must be met:

$$
\left|r_{1}-r_{2}\right|<\frac{\lambda}{2}
$$

In combination with Eq. 2.27 the condition reads $\frac{d L}{R}<\frac{\lambda}{2}$, and hence a maximal specimen size from where an interference pattern is still observable can be defined as

$$
d_{c, t r}=\frac{\lambda R}{2 L}
$$

This length is called coherence length. It can also be formulated using the angles shown in Fig. 2.9B, where $\alpha$ is the angle subtended by the source at the specimen $[36,46,130]$. With $\frac{L}{2 R}=\tan \left(\frac{\alpha}{2}\right) \approx \frac{\alpha}{2}$, the coherence length translates to

$$
d_{c, t r}=\frac{\lambda}{2 \alpha}
$$


Considering the angle $\beta$ which yields the coherence condition $L \beta \lesssim \frac{\lambda}{2}, L$ can be seen as the effective source size for coherent illumination. Using the above mentioned example with an acceleration voltage of $U=120 \mathrm{kV}$ and assuming an convergence angle of $2 \alpha=0.1 \mathrm{mrad}$, the transverse coherence length calculates to $d_{c, t r} \approx 33 \mathrm{~nm}$. Compared to the longitudinal coherence length, this value is one order of magnitude lower. Hence, the transverse coherence length is usually the limiting parameter concerning coherence lengths.

In electron beam physics, the transverse coherence length is often defined alternatively using the angular spread $\sigma_{\alpha}$ of the electron beam and a factor $\pi$, thus reading $[6,26$, 27]:

$$
d_{c, t r}=\frac{\lambda}{2 \pi \sigma_{\alpha}}
$$

By approximating $\sigma_{\alpha} \approx \sigma_{p_{r}} / p$ with the transverse momentum spread and using the de-Broglie relation, the spatial coherence length can be written as

$$
d_{c, t r}=\frac{\hbar}{\sigma_{p_{r}}}
$$

Additionally, in a beam waist the equation can be expressed in terms of the transverse normalized emittance $\left(\varepsilon_{n, r}\right)$ :

$$
d_{c, t r}=\frac{\hbar}{m c} \frac{\sigma_{r}}{\varepsilon_{n, r}}
$$

\subsection{Fundamentals in electron microscopy}

Electron microscopes were originally developed to overcome the resolution limit of light microscopes. As stated by Abbe in 1873, the resolution limit is at most half of the wavelength of the used light [131]. An improvement of the accuracy of this statement was given later incorporating the Rayleigh criterion. The resolution of a microscope is then given by the minimum distance at which two point like objects can be distinguished:

$$
d_{\min }=\frac{0.61 \lambda}{n \sin (\alpha)}
$$

where $\lambda$ is the wavelength used, $\alpha$ is the half angle subtended by the objective at the object, and $n$ the refractive index of the material between the object and the objective 
lens $[132,133]$. For example, when using green light (500 nm wavelength) and a numerical aperture of one $(N A=n \sin (\alpha)=1)$, this results in a resolution of about $300 \mathrm{~nm}$.

To overcome this limit, Ruska and Knoll proposed and subsequently built a microscope incorporating an electron beam instead of a light beam, using the electrons' wave-like characteristics first theoretically described by de Broglie [46]. By accelerating electrons with a voltage $U$, the de Broglie-wavelength can be expressed as

$$
\lambda=\frac{h}{\sqrt{2 m_{e} e U}} .
$$

Here, $h$ denotes the Planck constant, $m_{e}$ the electron rest mass and $e$ the elementary charge. Considering relativistic corrections for fast electrons, the formula reads

$$
\lambda=\frac{h}{\sqrt{2 m_{e} e U\left(1+\frac{e U}{2 m_{e} c^{2}}\right)}} .
$$

Following the approach of Abbe and Rayleigh, a large improvement of the resolution is to be expected from electron microscopes. With an accelerating voltage of $200 \mathrm{kV}$, a wavelength of $2.5 \mathrm{pm}$ is obtained, which is sufficient to resolve single atoms [46]. However, this resolution is deteriorated by aberrations of the electron lenses within the TEM and up to date, at most $50 \mathrm{pm}$ resolution is possible [46, 134].

In the following, a short introduction to electron microscopes is given. The general setup is presented as well as information about the main beam parameters.

\subsubsection{Electron microscope setup}

Similar to transmission and reflection light microscopes, electron microscopes exist for transmission and reflection mode as well. The transmission electron microscope (TEM) can be seen as a further development of the classical light microscope, albeit with a much smaller imaging wavelength due to the application of electrons. Since the electron beam is transmitted through the sample, it is used to study the internal structure of specimens. In contrast, scanning electron microscopes (SEM) can, in some sense, be seen as the equivalent of reflecting light microscopes and are used to study features at the surface of bulk materials [132]. 
A

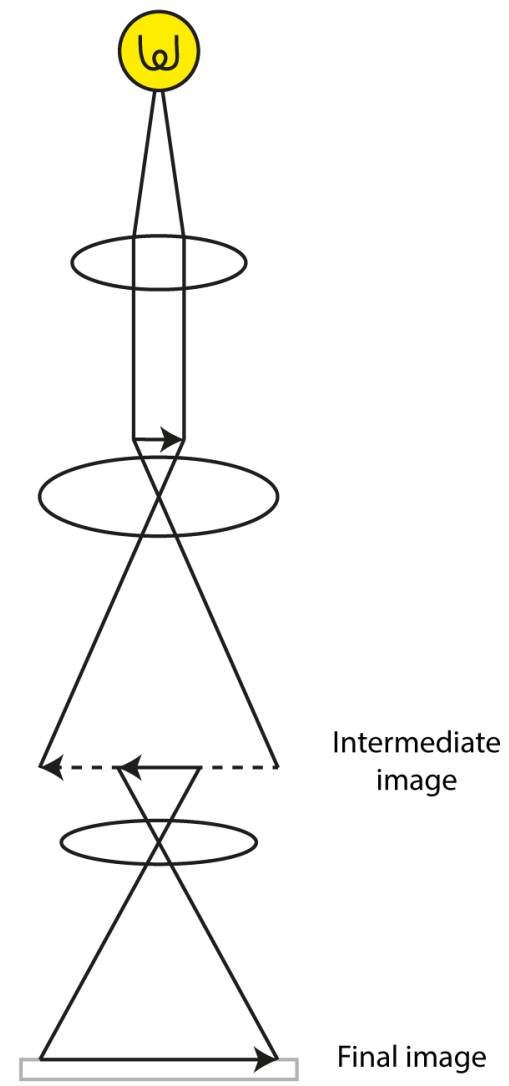

B

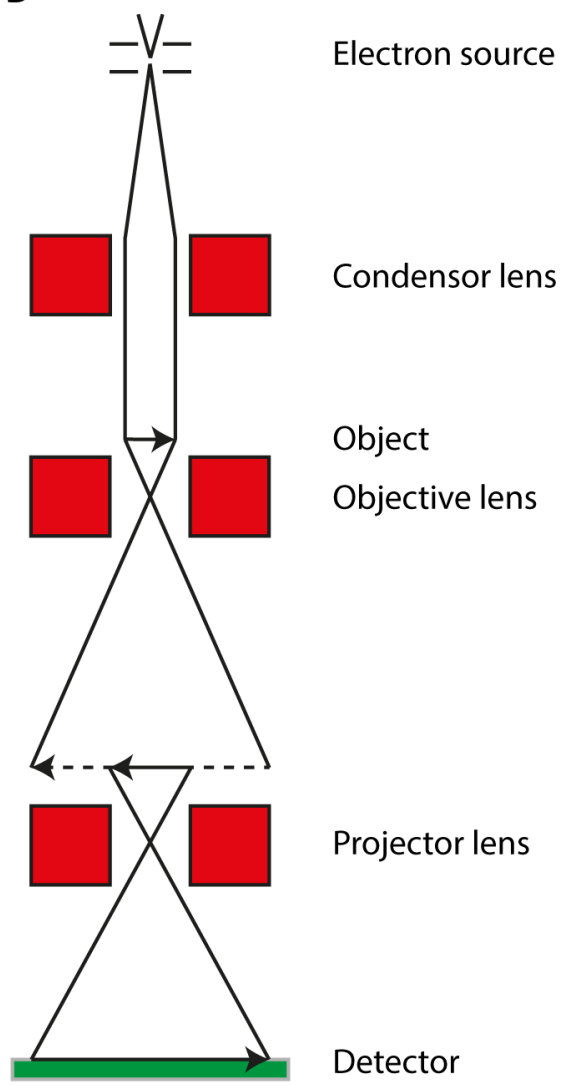

FIGURE 2.10: Light microscope setup. B: Rudimentary electron microscope with twostage magnification. Image adapted from Ref. [132].

The simplest electron microscope is built analogously to a (transmitting) light microscope using two lenses for image formation (see Fig. 2.10). This reduced setup is helpful to understand the basic concept of an electron microscope. First, electrons are generated in an electron source. The electron beam created is then focused onto the specimen by a condenser lens. An objective lens magnifies the electrons passing through the sample into an intermediate image. The latter is subsequently further magnified using a projector lens and forms the final image on the detector.

In contrast to the TEM just described, an SEM operates by scanning an electron beam over the specimen, recording the back-scattered electron signal or secondary electrons [45]. The spatial resolution is mostly determined by the focal size of the electron beam, which depends on the electron source size and the lens configuration [52]. Since the electrons in an SEM are not required to penetrate through the specimen, but to generate secondary electrons, the acceleration voltage is usually up to $30 \mathrm{kV}$, in contrast to a few hundred $\mathrm{kV}$ in a TEM. Fig. 2.11A shows a cross-section of a typical SEM setup. In 
A

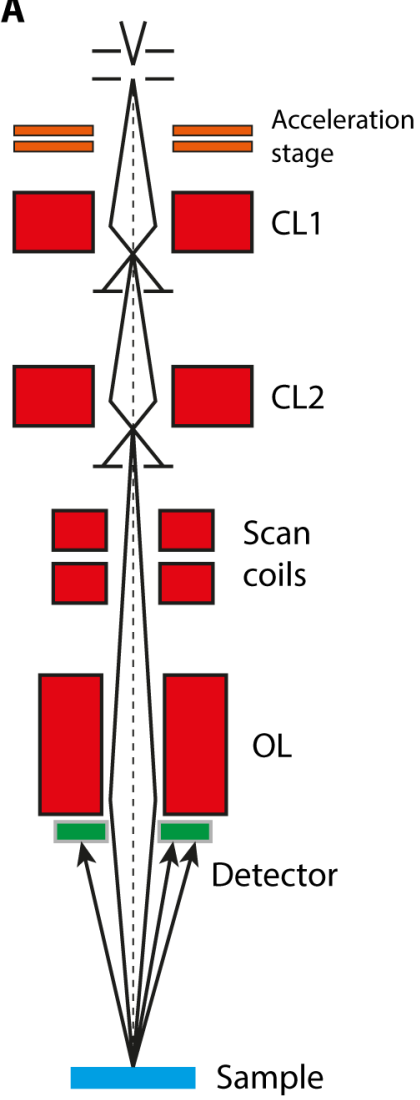

B

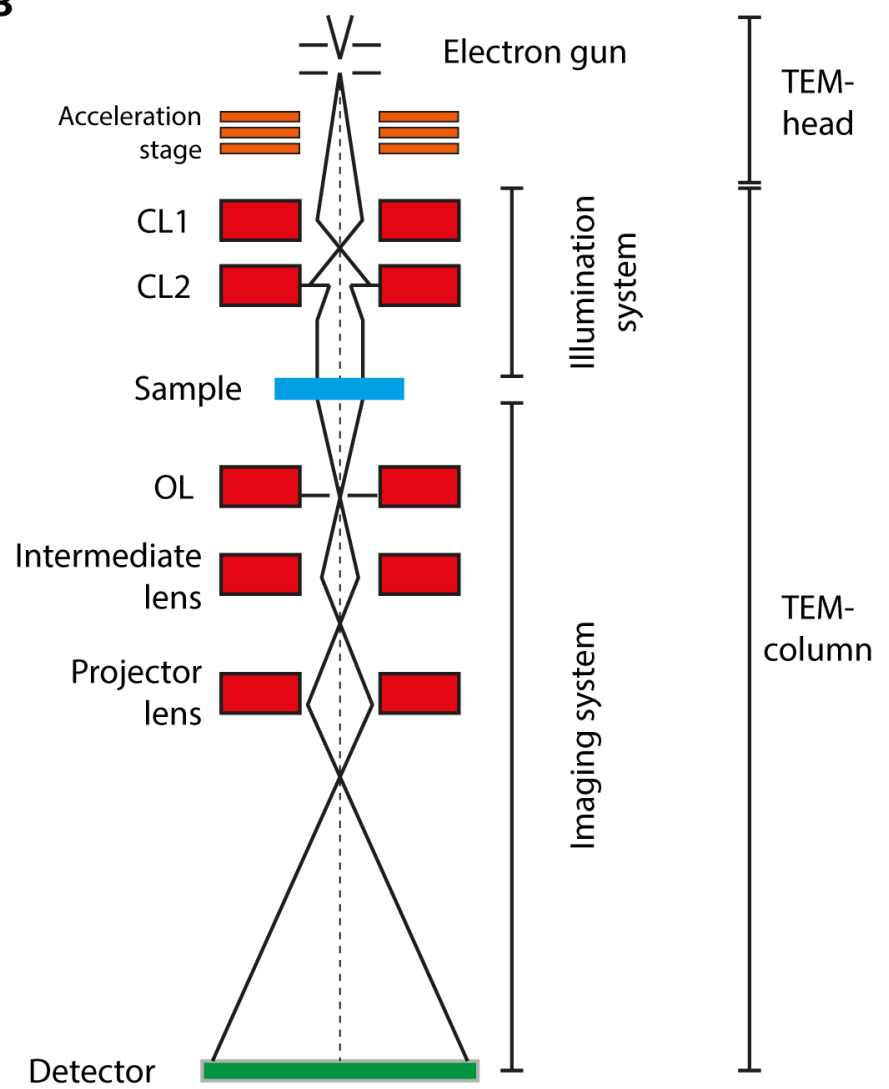

Figure 2.11: Schematic cross-sections of the two general electron microscopes. Condenser lens (CL) and objective lens (OL) are abbreviated. A: SEM with in-lens detector.

B: TEM with indicated divisions. Image adapted from Ref. [52].

Fig. 2.11B, a more involved setup of a TEM is shown.

Since the illumination part, being the section directly before the specimen, is in principle the same for an SEM and a TEM, and experiments were conducted using a TEM, only the TEM is discussed in the following.

The setup of a TEM is typically separated in divisions with regard to the electron lenses and deflection coils. As already mentioned, the section from the electron gun to the specimen is called the illumination system and usually does not include the electron gun itself. The part following the specimen up to the detector is called imaging system. The illumination system controls the so-called spot size and $\alpha$ selection in addition to the overall imaging mode (see Sec.2.5.2). The imaging system is responsible for the magnification, switching between imaging and diffraction mode in case of parallel illumination and detection of the signal of interest (see Sec. 2.5.3).

In terms of constructing a TEM, the whole illumination and imaging system is combined 
into the TEM-column (cf. Fig. 2.11B). Electrons entering the TEM-column are already fully accelerated. The part above ${ }^{5}$ the column contains the TEM-head and consists of the electron gun and the acceleration section. After the acceleration stage, deflection coils are implemented in order to couple the electron beam into the TEM-column. The setup of different electron guns is presented in Sec. 2.3.

\subsubsection{Illumination system}

The main purpose of the illumination system is to transfer the electrons from the source to the specimen and to shape the electron beam in the process. Two main operation modes are distinguished: parallel beam and convergent beam mode. In the first case, imaging and selected-area diffraction are achieved. The second mode, in which the beam is focused onto the sample, is mainly used for scanning TEM imaging (STEM) or convergent beam diffraction (CBED).

The TEM model used in this thesis (Chap. 7) is a JEOL JEM-2100F microscope. The ray diagrams will be shown with regard to the setup of this particular model. As already mentioned, the illumination system allows the user to choose, which and under what conditions electrons from the electron gun impinge on the specimen.

The two main modes are shown in Fig. 2.12, where Fig. 2.12A-C depicts the parallel illumination mode and Figure Fig. 2.12D-E the convergent beam mode.

In parallel mode, the upper polepiece of the objective lens is held at a constant current. Adjustments are done by the condenser lens $\mathrm{CL}^{6}{ }^{6}$ and the condenser mini-lens (CM), which have to produce an image of the gun crossover at the front-focal plane of the upper polepiece in order to obtain parallel illumination at the specimen. However, the beam will never be exactly parallel and is therefore usually underfocused (focus spot far below the specimen), which leads to a convergence angle $\alpha$ typically in the range of $0.1 \mathrm{mrad}$ [46]. By adjusting the CL3 lens, the electron beam can be (de-)focused at the specimen. This can, in principle, be used to set the illuminated area in parallel beam mode. However, in order to regain parallel illumination, either the condenser lens aperture (CL aperture / CLA) or the $\alpha$-selector has to be adjusted accordingly. The

\footnotetext{
${ }^{5}$ Almost all TEMs are built vertical. This is mainly because when assembling the individual parts of a TEM, they have to be aligned precisely and must not shift.

${ }^{6}$ In the JEOL JEM2100F microscope, the second condenser lens is named CL3. However, it acts like the second condenser lens described in literature, e.g. Ref. [46, 133].
} 


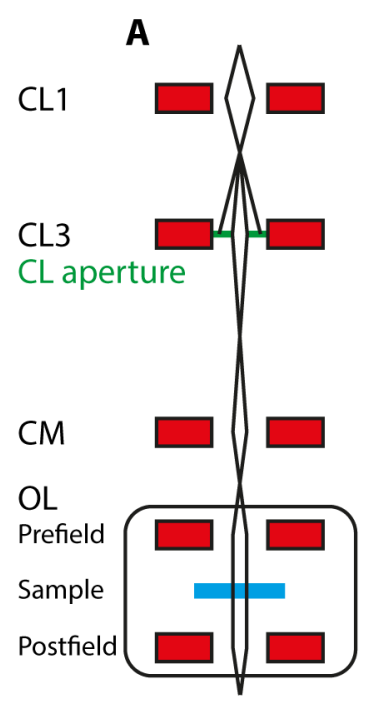

$\alpha=1$

spot $=1$
B

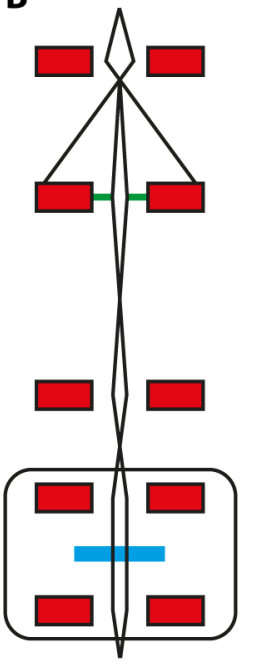

$\alpha=1$

spot $=2$

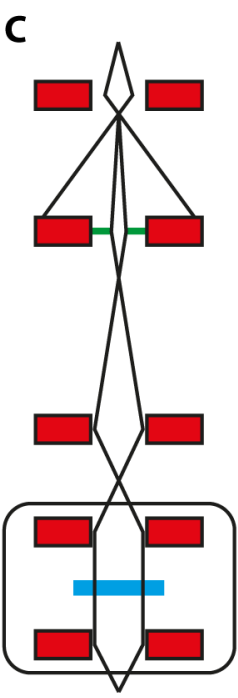

$\alpha=2$

spot $=2$
D

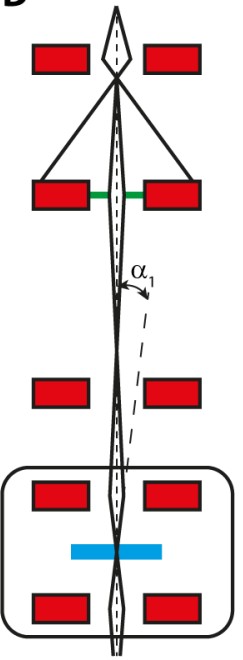

$\alpha=1$

spot $=2$
$\mathbf{E}$

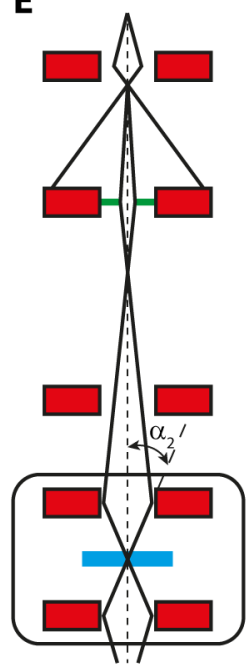

$\alpha=2$

spot $=2$

Parallel beam mode

Figure 2.12: Simplified ray diagrams for parallel (A-C) and convergent (D-E) beam mode and different settings of the illumination system.

CL3 lens can (in parallel mode) also be adjusted in such a way that the beam is focused onto the specimen. This mode will be used to determine beam parameters in Chap. 7 .

In contrast to the parallel mode, in convergent beam mode the CM lens is tuned down (or turned off) and the upper objective polepiece is used to focus the electron beam onto the specimen. In convergent beam mode, the spot- and $\alpha$-selector directly influence the beam properties. The function of the two are shortly introduced in the following.

\section{Spot selection}

The first condenser lens (CL1) forms an image of the gun crossover as depicted in

Fig. 2.12. The strength of the CL1 lens determines the number of electrons that are cut at the CL-aperture. This is usually called spot selection and can be adjusted in discrete steps in the JEOL microscope. Spot 1 marks a weak CL1 lens and thus a higher electron current and larger source size, whereas spot 5 stands for the highest CL1 strength and therefore the lowest source size (cf. Fig. 2.12A, B). Fig. 2.12A-C shows the parallel beam operation mode (regular TEM mode), however, the spot selection works in the same way for the convergent beam mode (Fig. 2.12D-E). Furthermore, for convergent beam mode the spot selection actually determines the spot size of the electron beam on the specimen due to the variation of the (virtual) source size. 


\begin{abstract}
Alpha selection
Independent of the spot selection, an $\alpha$-selector can be set, even though the convergent angle $\alpha$ at the specimen depends, e.g., also on the CL aperture. For parallel illumination, a different value of the $\alpha$-selector leads to a change of the CL3 lens strength. By simultaneously changing the CM lens strength, parallel illumination of the specimen is preserved. In case of a convergent beam, the $\alpha$-selection directly leads to a different convergent angle at the specimen (cf. Fig. 2.12D, E).
\end{abstract}

\title{
2.5.3 Imaging system
}

After passing through the specimen, electrons are collected by the (lower polepiece of the) object lens. A diffraction pattern is created at the (back-) focal plane of the objective lens, and an image is formed in the image plane (see Fig. 2.13). In order to be able to observe a diffraction pattern on the detector, the back-focal plane of the objective lens has to be the object plane of the intermediate lens (Fig. 2.13A). On the other hand, in order to obtain an image of the specimen on the detector, the object plane of the intermediate lens has to coincide with the image plane of the objective lens (Fig. 2.13B). The projector lens in both cases subsequently magnifies the pattern mapped by the intermediate lens. 
A

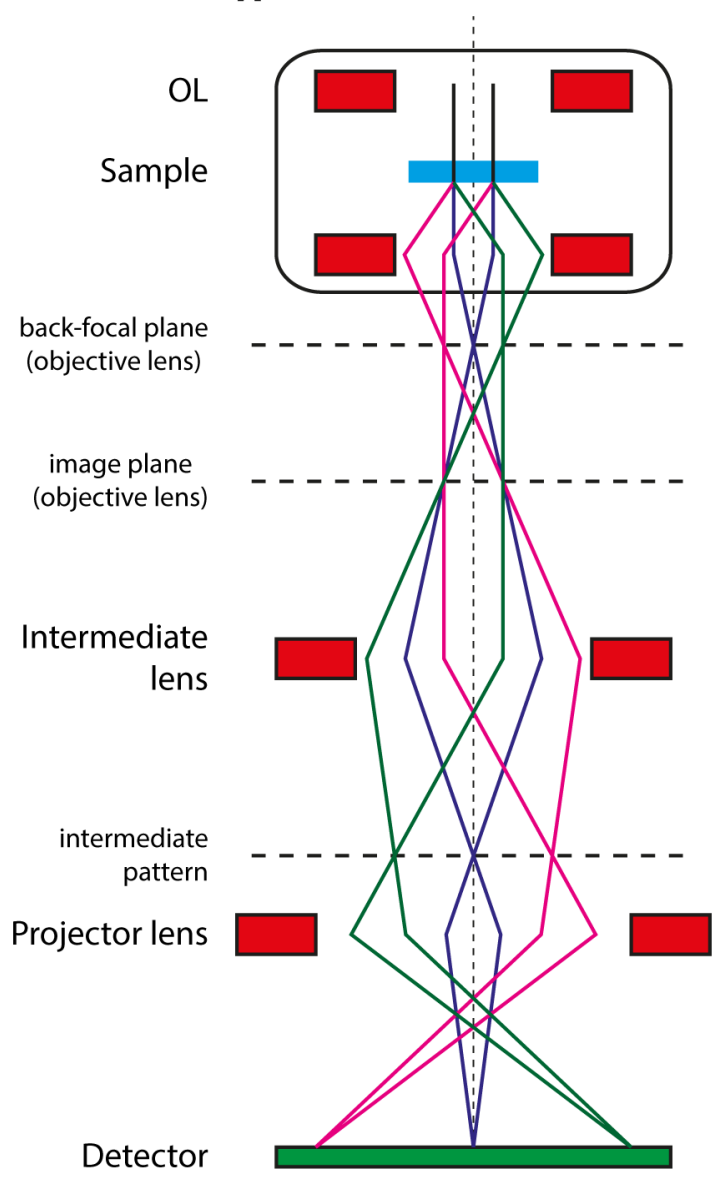

B

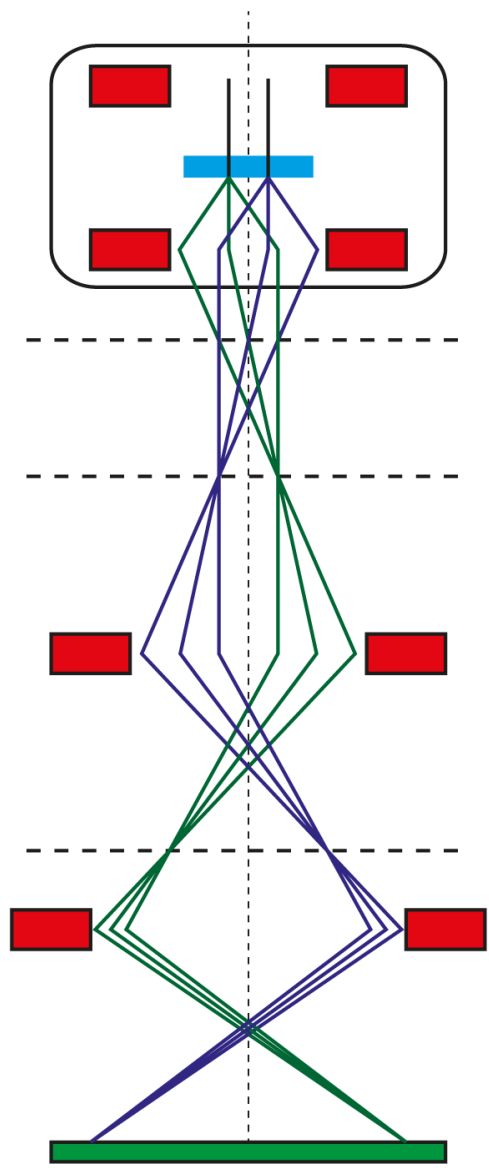

Figure 2.13: Simplified diagram of the two modes of the imaging system. A: Diffraction mode. The diffraction pattern of the specimen forms at the back-focal plane of the objective lens. This plane acts as the object plane of the intermediate lens and is subsequently projected onto the detector. B: Imaging mode. The object plane of the intermediate lens is set to the image plane of the objective lens. Now the magnified image is visible at the detector. 


\section{Chapter 3}

\section{Prerequisites for simulation}

This chapter introduces the numerical simulation methods used. First, a description of the employed numerical finite element method is given, and the principle of the numerical simulation is explained. Subsequently, the electrostatic potential landscape in the vicinity of the emitter is computed and discussed with respect to its influence on electron emission. The electron emission characteristics is described in this thesis by means of a single parameter $\Gamma$, which is introduced in this chapter.

\subsection{Numerical simulation method}

For a better understanding of the measurements and to gain insight into the electron propagation after emission, electron gun configurations are simulated using a finite element analysis. From the obtained electron trajectories, one can deduce beam properties such as spatial distribution, emittance or pulse duration.

The finite element method (FEM) is a numerical technique for solving partial differential equations. It provides an approximate solution to boundary-value problems [135, 136]. The accuracy can be enhanced at the expense of calculating time. Since FEM simulations offer the possibility to solve complex problems, they enjoy great popularity in physics and engineering. However, a parametric analysis is not readily possible. At most, a variation of parameters can be obtained at the cost of additional computation time.

The basic idea of FEM is to divide the underlying geometry into a number of smaller volumes, the so-called mesh cells. These volumes have a finite size and can be described 
by a (finite) number of parameters. Naturally, the solution should converge to a value which is independent of the discretization for an increasingly fine mesh. As the computational cost rises with the number of elements in the simulated volume, the mesh has to be made as coarse as possible within the desired accuracy. For a more detailed description of the finite element method, the reader is referred to the literature, e.g. Refs. [135-137].

In the applied FEM simulations the Laplace equation $\Delta U(r, \varphi, z)=0$ needs to be solved in order to obtain an approximation for the electrostatic potential $U(r, \varphi, z)$. Due to the cylindrical symmetry of the problem (see Fig. 3.1), the electrostatic potential is independent of $\varphi: U=U(r, z)$ and the Laplace equation is reduced to

$$
\Delta U=-\frac{1}{r} \frac{\partial}{\partial r}\left(r \frac{\partial U}{\partial r}\right)-\frac{\partial^{2} U}{\partial z^{2}}=0 .
$$

The boundary conditions satisfy the Dirichlet criterion for electrode elements and the tip emitter, since the potential $U$ is fixed at that region: $U(r, \varphi, z)=U_{\text {electrode. If the }}$ gradient of the potential with respect to the surface vanishes, i.e. in every remaining surface, von Neumann boundary conditions are used: $\partial U(r, \varphi, z) / \partial \mathbf{n}=0$. Here, $\mathbf{n}$ denotes the normal vector of the respective boundary.

The size and geometric structure of the needle emitter is based on typical shapes of tungsten tips employed in the measurements (cf. Sec. 4.1). Not only the apex diameter, but also the overall shape of the tip shank is adopted. The construction of the geometry layout including the gun assembly and vacuum chamber is done using Autodesk Inventor $^{1}$. The mesh is then generated by the software Gmsh using the "MeshAdapt" algorithm $^{2}$ (see Fig. 3.1). To reduce the number of elements within the mesh and therefore to reduce computational cost, areas with different precision are defined for microscopic and macroscopic scales. This results in a fine mesh at the tip apex (microscopic scale) and along the shaft, a moderate discretization along the remaining anodes (suppressor, extractor) and less precision between the electron gun and the detection plane (macroscopic scale). For maximum performance, the mesh size and temporal step size have been optimized before starting the complete simulations (see Fig. 3.1).

\footnotetext{
${ }^{1}$ More about Inventor can be found at http://www . autodesk. com/products/inventor.

${ }^{2}$ Information about Gmsh and the MeshAdapt algorithm can be found at http://geuz.org/gmsh/ doc/texinfo/gmsh.txt.
} 


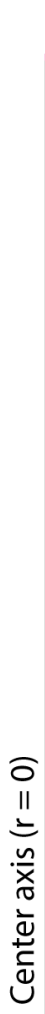

A

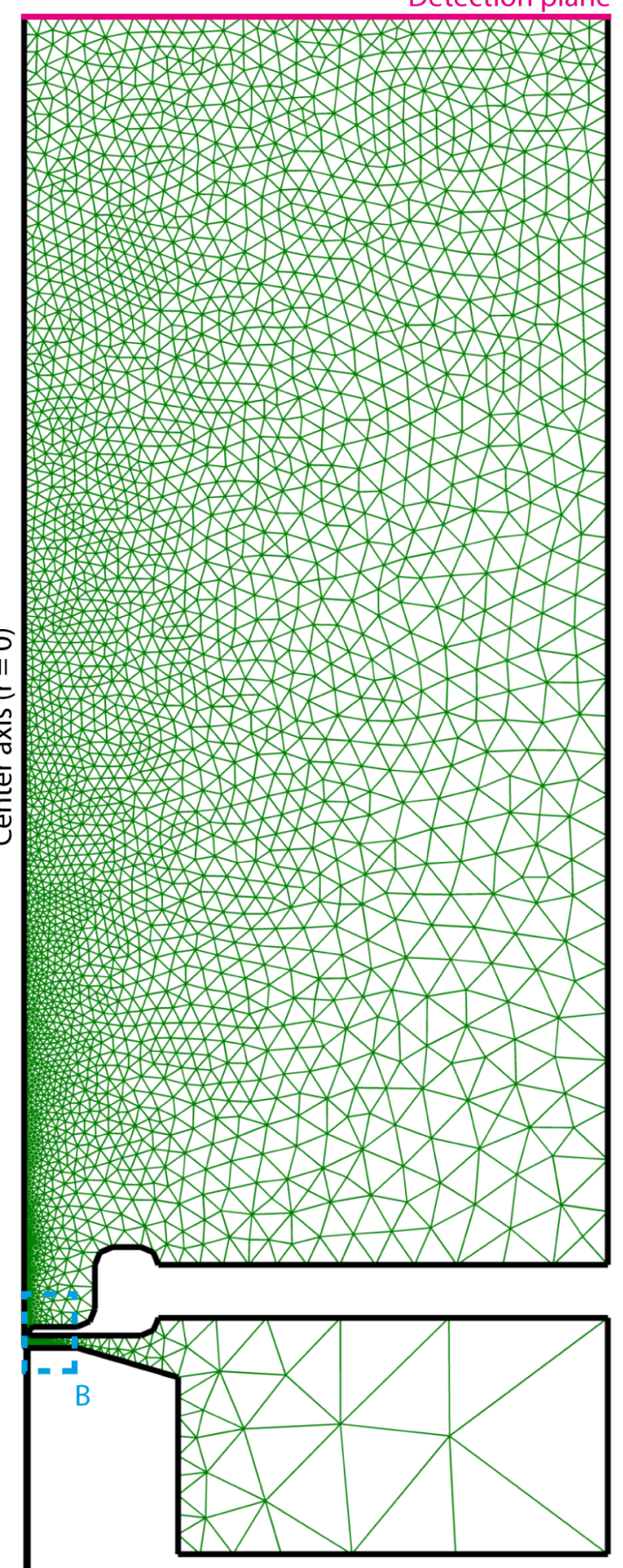

Detection plane

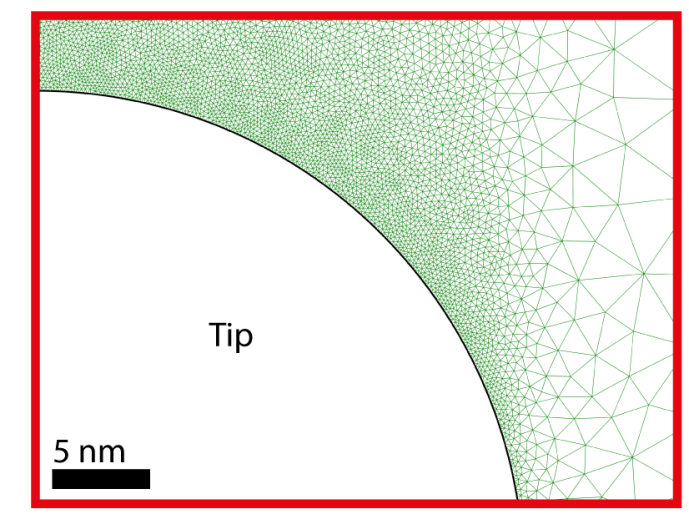

B

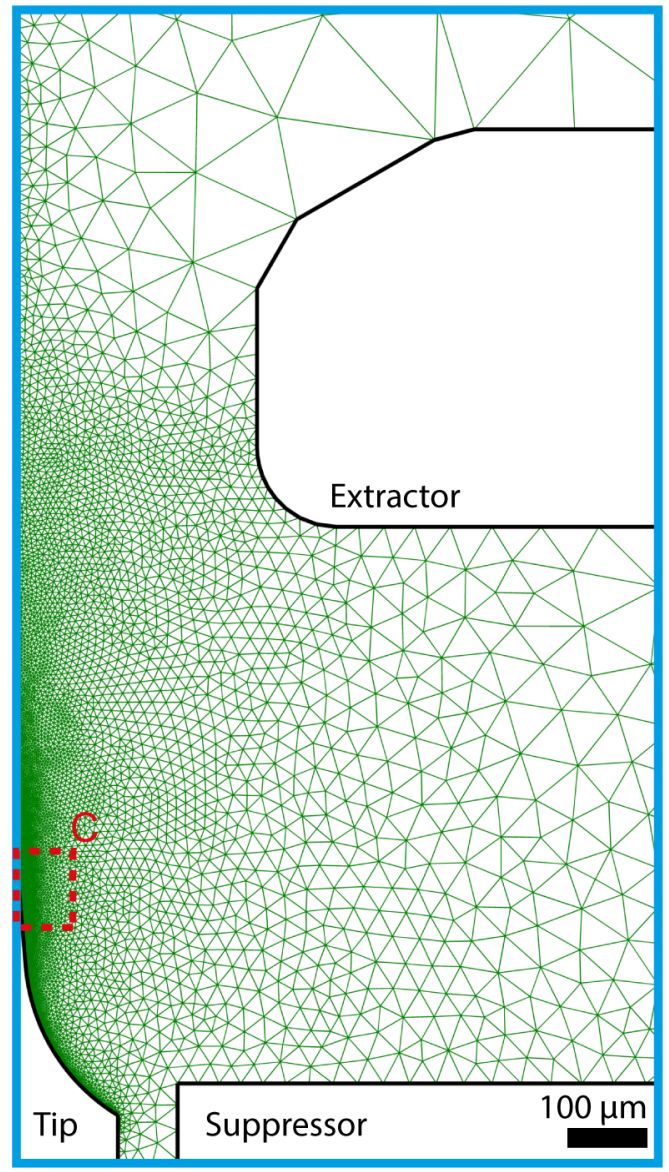

$3 \mathrm{~mm}$

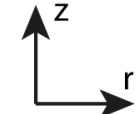

FiguRE 3.1: Generated mesh used for FEM simulations at different magnifications. The left edge in all three figures corresponds to $r=0$. 
The FEM solution is computed using MathWorks Matlab ${ }^{3}$ software. The general implementation of the Matlab script was programmed by Felix Schenk ${ }^{4}$. The accuracy of the program was verified and compared to simulations using Comsol Multiphysics ${ }^{5}$ in Refs. [34, 35].

Using the computed electric potential, electron trajectories can be classically simulated by solving the equation of motion

$$
\frac{d^{2} \mathbf{r}}{d t^{2}}=\frac{e}{m_{e}}\left(\begin{array}{l}
\partial U(r, z) / \partial r \\
\partial U(r, z) / \partial z
\end{array}\right)
$$

A cylindrical symmetry is assumed as well as no out-of-plane momentum to substantially reduce calculation time. Computations are done for single electrons within the Matlab framework using a Runge-Kutta algorithm. The single electron approach is reasonable for several reasons. In the experiments conducted within this work, the electron count rate is at maximum on the order of a few tens of electrons per pulse. In addition, in prior experiments for similar geometries, space charge effects were not observed [29]. As can be seen later in Sec. 4.6, space charge seems to be negligible for the experimental conditions used here as well. Hence, electron-electron interactions are not taken into account, and the electron trajectory depends only on initial parameters and the calculated electric field. Parameters include the kinetic energy of the electron, the starting point on the tip surface and the starting angle with respect to the tip surface. The calculation of an electron trajectory is finished whenever it impinges on a boundary of the geometry or when it crosses the detection plane at the end of the simulation area. For further use, the velocity, spatial coordinates and the time of flight are saved for every trajectory.

\subsubsection{Probability density function}

For simplicity, a smooth tip surface and a rotationally symmetric emitter is assumed. Furthermore, effects induced by the electric field of the ultrafast laser pulse are not taken into account. Moreover, further simplifications are necessary, as the exact laser wavelength, laser intensity, and applied potential can significantly affect the electron

\footnotetext{
${ }^{3}$ See http://de.mathworks.com/products/matlab/ for product information.

${ }^{4}$ In cooperation with the group of Thorsten Hohage from the Institute of Numerical and Applied Mathematics (University of Göttingen). More information can be found in Ref. [17, 34, 35, 138].

${ }^{5}$ Information about Comsol Multiphysics can be found at http://www.comsol.com/ comsol-multiphysics.
} 

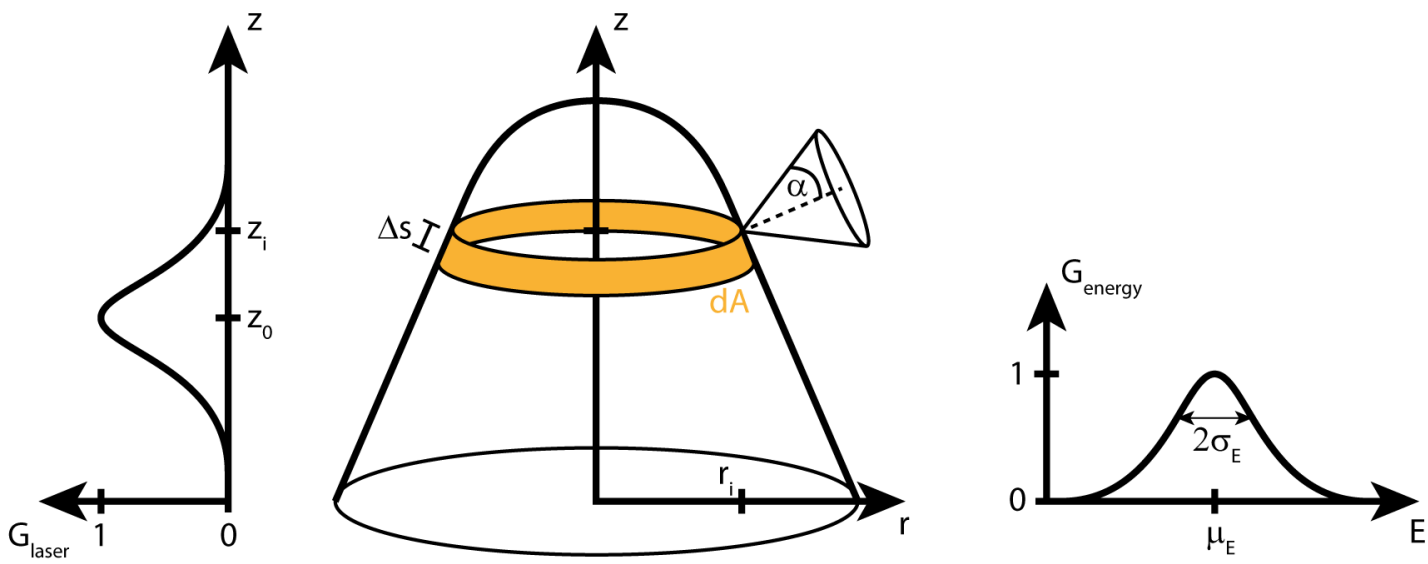

Figure 3.2: Simulated tip emitter and probability density functions.

trajectory and hence the spatial and temporal shape of the electron beam. In the following, the assumptions on initial parameters are described.

The initial kinetic energy of the emitted electrons is assumed to be a Gaussian distribution, in reasonable agreement with various experiments concerning the photoemission in similar setups $[28,34,35,91,138-141]$. The distribution reads

$$
G_{\text {energy }}(E)=G_{0, E} \cdot \Theta(E) \cdot \exp \left(-\frac{\left(E-\mu_{E}\right)^{2}}{2 \sigma_{E}^{2}}\right)
$$

where $G_{0, E}$ ensures normalization, $\mu_{E}$ denotes the mean energy and $\sigma_{E}$ the standard deviation of the kinetic energy. The Heaviside function $\Theta(E)$ ensures that the kinetic energy is larger than zero. Initial kinetic energies of exactly zero are neglected, since they cause computational issues in the simulation. Instead, an energy of $E=10^{-4} \mathrm{eV}$ is used in place of zero kinetic energy, which is negligible compared to occurring energies on the order of $1 \mathrm{eV}$.

Concerning the emission angle $\alpha$ (measured with respect to the surface normal), a homogeneous distribution is assumed. Dowell et al. considered the photoelectron generation in terms of emission angles similar to an optical refraction process [49]. Hence, the emission angle is not restricted and can have values of $-\pi / 2 \leq \alpha \leq \pi / 2$, where $\alpha=0$ corresponds to the surface normal. In this work, for the same reasons as for the initial kinetic energy of zero, the maximum angle is reduced to $\pm \pi / 2 \mp 0.01$ to avoid numerical problems. 
The tip shape used for simulations is modeled according to SEM images of tungsten tips used in the experiments. To account for the different length scales on the emitter tip, the starting points of calculated photoelectron trajectories are not evenly distributed across the emitter surface. Instead, a high density of starting points is used at the tip apex compared to the tip shaft. This results in the same order of magnitude of initial spatial coordinates at the tip shaft and apex. Normalization of the emission site is given in terms of emission area taking into account the three-dimensional shape of the emitter:

$$
G_{\text {area }}\left(\mathbf{r}_{i}\right)=G_{0, r_{i}} 2 \pi r_{i} \Delta s
$$

Here, $r_{i}$ is the initial radial component of the electron trajectory in cylindrical coordinates, and $\Delta s=\sqrt{\Delta r_{i}^{2}+\Delta z_{i}^{2}}$ is the section of the tip surface between two adjacent initial spatial coordinates.

In a prior work, the solid angle of emission from a gold tip, for multi-photon emittion with $800 \mathrm{~nm}$ laser light, was found to be $\Omega=0.28 \mathrm{sr}$ [29]. One could argue, that the calculation of electron trajectories from a confined region on the tip apex surface is sufficient for the simulation of an electron gun. However, the above mentioned experiments were performed without additional electrodes (i.e. suppressor, extractor), and the nonlinearity of the emission process was higher. The higher nonlinearity itself leads to a lower solid angle of emission, since the necessary field strength for electron emission is more confined to the tip apex (see Sec. 2.3.1). Therefore, in the simulations computed within this work, no further assumptions are made with regard to the emission site.

Further selection of the emission site is performed in terms of excitation due to a laser pulse. Here, a Gaussian distribution along the $z$-axis models the laser position and intensity. Electrons originating from the center of the laser pulse $\left(z_{0}\right)$ are therefore emitted with a probability of one, and all other electrons with a probability corresponding to the Gaussian distribution of the laser pulse:

$$
G_{\text {laser }}\left(z_{i}\right)=G_{0, z_{i}} \cdot \exp \left(-\frac{\left(z_{i}-z_{0}\right)^{2}}{2 \sigma_{z}^{2}}\right)
$$

In the simulations carried out within this work, the electron trajectories are first calculated for a range of starting points along the tip surface, initial energies and emission angles. Normalization and weighing is implemented afterwards using the combination 


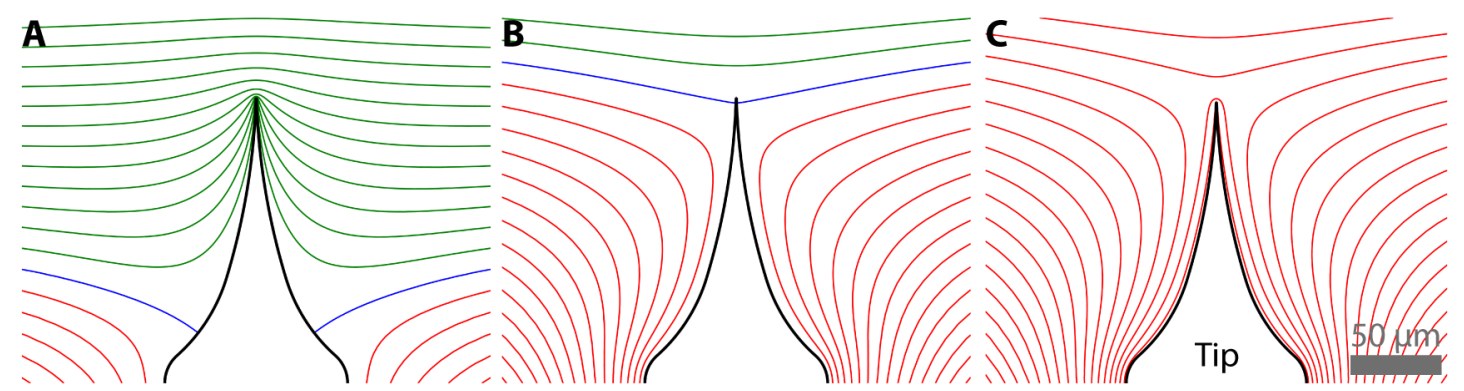

Figure 3.3: Equipotential lines for different voltage settings at extractor, suppressor and tip electrode: A: $\Gamma=0.58, \mathrm{~B}: \Gamma=1.15, \mathrm{C}: \Gamma=1.32$ (see Eq. 3.7 for the definition of $\Gamma$ ). Green (red) lines depict those with a higher (lower) potential than the emitter tip, the equipotential line with the same value is plotted blue.

of the just described probability density functions:

$$
G\left(E, \mathbf{r}_{i}, z_{i}\right)=G_{0} \cdot \Theta(E) \cdot \exp \left(-\frac{\left(E-\mu_{E}\right)^{2}}{2 \sigma_{E}^{2}}-\frac{\left(z_{i}-z_{0}\right)^{2}}{2 \sigma_{z}^{2}}\right) \cdot 2 \pi r_{i} \Delta s
$$

\subsection{Electrostatic landscape and $\Gamma$-parameter}

In this section, the electrostatic field distribution around the tip is modelled for different voltage configurations at the gun assembly. A parameter $(\Gamma)$ is introduced, in order to estimate for which voltage settings electrons can be emitted from a metal tip embedded in such a suppressor and extractor electrode. Obviously, the potential around the tip has to be more positive than the tip potential itself, otherwise electrons would be accelerated back to the tip even if an emission were possible.

Fig. 3.3 shows equipotential lines for different voltages at the suppressor, extractor and tip. Green lines correspond to electric potentials higher (more positive) than the potential of the tip emitter and red (blue) lines to those which are lower (equal).

The point on the tip, where the equipotential line from the vicinity of the emitter coincide with the surface is called the cutoff-point. It marks the transition of the electric force pointing away from the tip emitter to pointing towards the tip emitter. For the latter case, electron emission is strongly suppressed.

Three different emission regimes can be determined. The first regime is shown in Fig. 3.3A. The applied voltages are $U_{\text {tip }}=-800 \mathrm{~V}, U_{\text {sup }}=-1000 \mathrm{~V}$ and $U_{\text {ext }}=0 \mathrm{~V}$. Here, the cutoff point is on the shaft, "far" away from the tip apex region. Hence, the electric field at the apex is enhanced, as expected from a metal tip (lightning rod effect) 
A

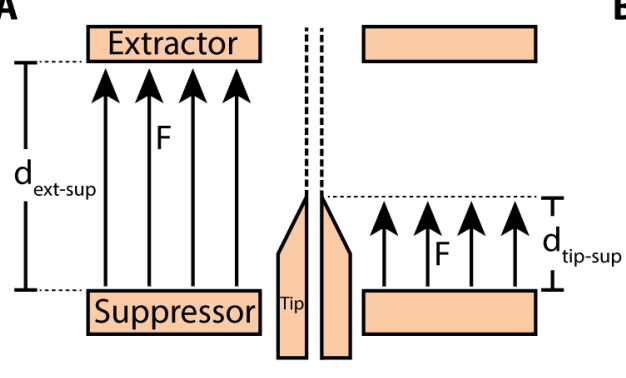

B

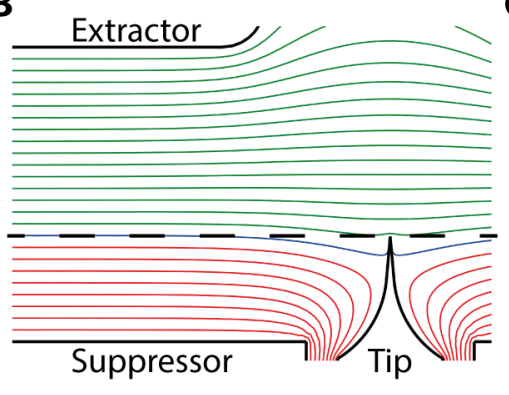

C

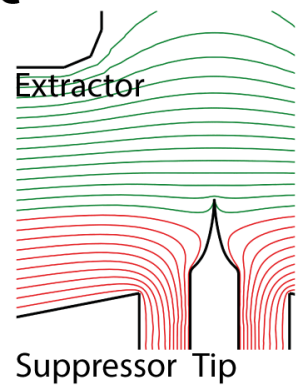

Figure 3.4: Sketch of the simplified plate capacitor model. B: Potential landscape illustrating the distortion of the potential lines in the vicinity of the tip emitter compared to the plate capacitor situation. C: Same as B, but for a different suppressor shape.

and electron emission can efficiently occur. Furthermore, with the enhanced field, electrons are quickly accelerated away from the tip, which leads to a suppression of space charge mediated beam broadening and, since the final velocity is reached more quickly, is also expected to reduce the pulse duration [35, 138, 142]. In this setting, electron emission is possible even at the tip shaft. This situation differs when the cutoff point approaches towards the tip, e.g., by increasing the tip voltage (Fig. 3.3B). The static field enhancement decreases, and electron emission from the tip shaft is suppressed due to the electric force pointing towards the surface. In the third case, the whole tip is surrounded by equipotential lines with lower potential than that of the emitter (see Fig. 3.3C). Therefore, no electron emission can occur.

To be able to distinguish the three regimes mentioned above, one can compare the situation of the electrode assembly to a simple parallel plate capacitor (cf. Fig. 3.4A). When the potential of the tip has the same value as the equipotential line at the height of the apex in a plate capacitor formed by the suppressor and extractor, the second of the above-mentioned scenarios is applicable. To reach the first regime, the equipotential line in a suppressor-extractor capacitor with the same potential as the tip must lie closer to the suppressor (and closer to the exractor for the third case).

In other words, one compares the electric field of a capacitor with voltages and distance of suppressor and extractor electrode to the fields formed by the tip and suppressor. 
The ratio $\Gamma$ between the two electric fields is used to distinguish between the emission regimes throughout this work.

$$
\Gamma=\frac{U_{\text {tip }}-U_{\text {sup }}}{d_{\text {tip-sup }}} / \frac{U_{\text {ext }}-U_{\text {sup }}}{d_{\text {ext-sup }}}=\frac{U_{\text {tip }}-U_{\text {sup }}}{U_{\text {ext }}-U_{\text {sup }}} \cdot \frac{d_{\text {ext-sup }}}{d_{\text {tip-sup }}} .
$$

Values of $\Gamma<1$ correspond to the field-enhanced regime, with possible emission from the tip shaft; $\Gamma \approx 1$ denotes the regime with emission only from the - not enhanced - apex region, and for $\Gamma>1$ electron emission is suppressed. This holds under the simplified assumption of a plate capacitor. However, for the geometries used in simulation and experiment, the transition occurs at values of $\Gamma \approx 1.2$, due to the distortion of the potential landscape by the tip electrode (visible in Fig. 3.4B).

This distortion, however is also largely influenced by the shape of the electrodes. For a different suppressor shape (see Fig. $3.4 \mathrm{C}$ ), this transition occurs at $\Gamma \approx 1.4$.

Results of the simulated trajectories and calculated beam properties can be found in Chap. 5 and Chap. 6, respectively. 



\section{Chapter 4}

\section{Experimental setup and first}

\section{measurements}

In this chapter, the experimental setup is explained, including the emitter preparation and alignment of the electron gun. An illustration of the electron gun is shown in Fig. 4.1. A tungsten tip is built into a suppressor-extractor unit. Preliminary measurements are shown including static tunnel emission, the photoelectric yield measured with an electrometer, as well as the nonlinearity of the power dependency of the electron emission process.

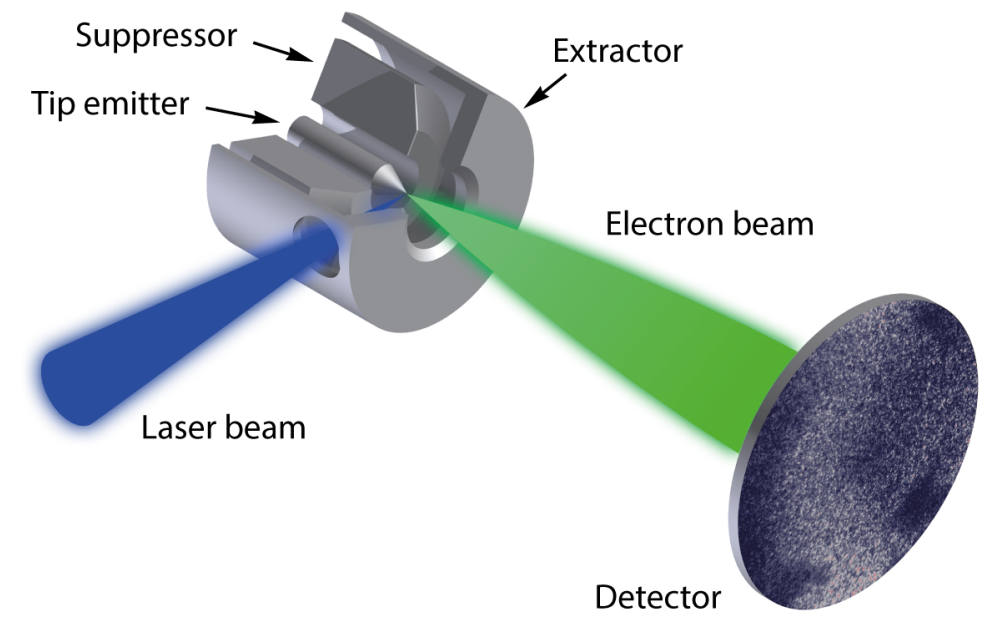

Figure 4.1: Schematic of the setup of the electron gun. Picture is not to scale. 

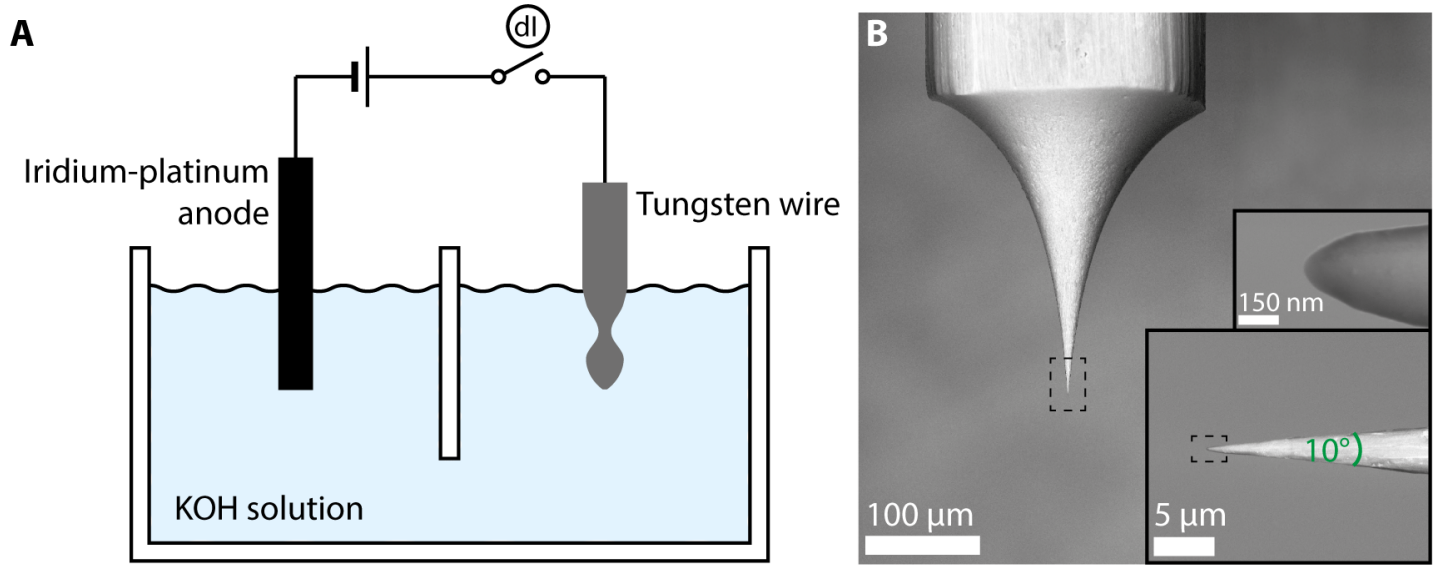

Figure 4.2: Tungsten tip fabrication. A: Illustration of the chemical etching setup. B: SEM image of a tungsten tip. Insets show higher magnification.

\subsection{Fabrication of sharp tungsten tips}

For building an ultrafast electron gun, a sharp metal tip with a radius of curvature of a few tens to a hundred nanometers is necessary. Typically, the tip material is tungsten, due to its high melting temperature, mechanical strength, good electric and thermal conductivity and low vapor pressure. On the downside, oxidation of the tungsten occurs, which requires storage under appropriate vacuum conditions. The shaping of tungsten needle emitters is performed by electrochemical etching with a method derived from Ref. [143]. Fig. 4.2A shows a sketch of the etching setup. The plain tungsten wire $(250 \mu \mathrm{m}$ diameter) is mounted into a holder and submerged by $3 \mathrm{~mm}$ into a $3.5 \% \mathrm{~mol}$ aqueous potassium hydroxide $(\mathrm{KOH})$ solution. A DC voltage of $6.5 \mathrm{~V}$ is applied between an iridium-platinum anode and the tungsten wire, which leads to a tapering of the wire. The process continues until the weight of the lower part of the wire cannot be supported anymore by the notched region and falls off. At this point, the etching current drops significantly and triggers an electric circuit to shut off the external voltage, thus stopping the etching process. The newly formed tip is washed in ethanol and deionized water. A first characterization is conducted under a light microscope to check whether the tip is straight and symmetrically formed. Subsequently, an inspection in a scanning electron microscope (SEM) reveals the radius of curvature (Fig. 4.2B). 

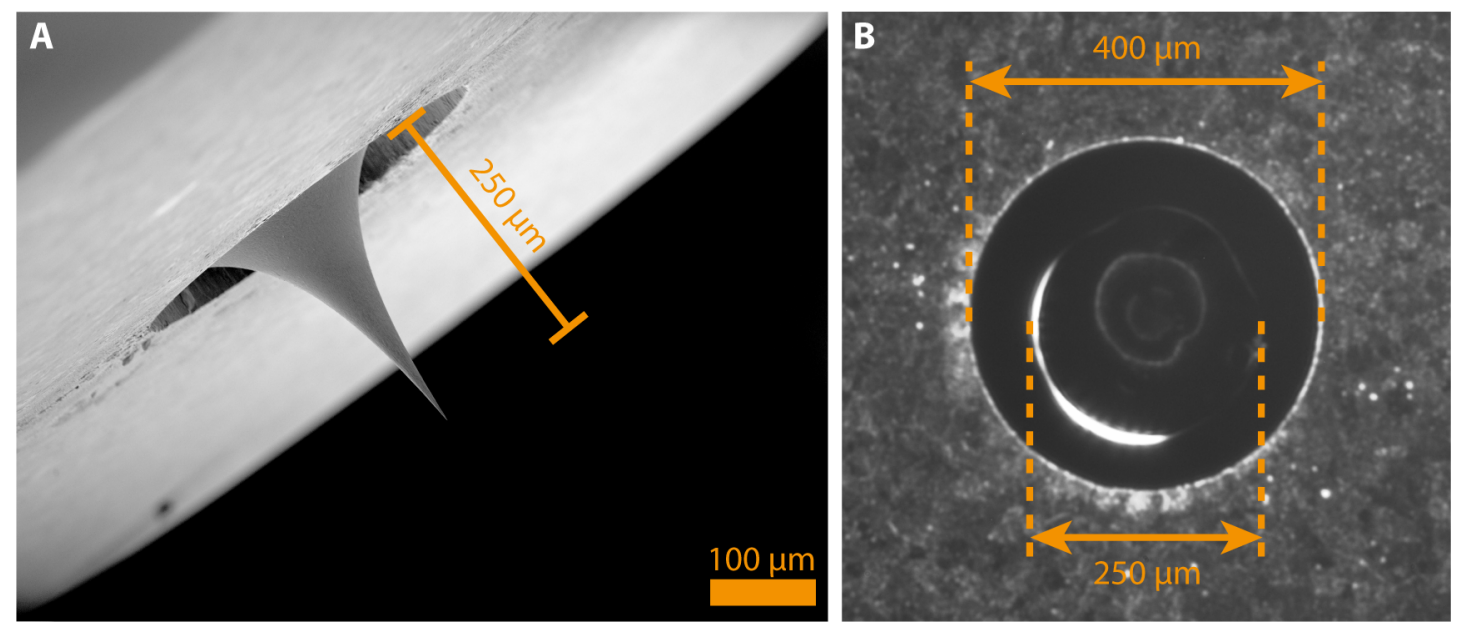

Figure 4.3: A: SEM image of the suppressor with an installed tip. B: Light microscope image showing a correctly aligned tip (top view). Note that the irregularly glowing surfaces at the tip are a result of light reflection from the single-crystalline wire.

\subsection{Gun assembly alignment}

After preparation, tips with a well-formed, sharp apex are mounted into the suppressor electrode. To this end, a self-made tip holder is used, which is then inserted into the suppressor. Symmetric alignment is very important in this step. A rotation mount is used to ensure the orientation of the tip, and, if necessary, the wire is bent accordingly. The distance between tip apex and suppressor surface is inspected under a light microscope with integrated scale. Changing the apex-suppressor displacement and subsequent realignment of the orientation is carried out, until the result is satisfactory (cf. Fig. 4.3B). The desired distance, by which the tip protrudes the suppressor, is $250 \mu \mathrm{m}$. In order to limit the duration of the alignment process to acceptable time-scales, a deviation of up to $30 \mu \mathrm{m}$ is considered to be acceptable.

The suppressor with the tip, aligned in the manner described, is thereafter mounted into the gun holder. A uniaxial light microscope is used to mount and correctly adjust the extractor electrode in such a way as to concentrically overlay apertures and tip apex.

\subsection{Experimental setup}

In order to drastically reduce the complexity of the experimental setup compared to a TEM environment, electron source characterizations are performed in a small vacuum 


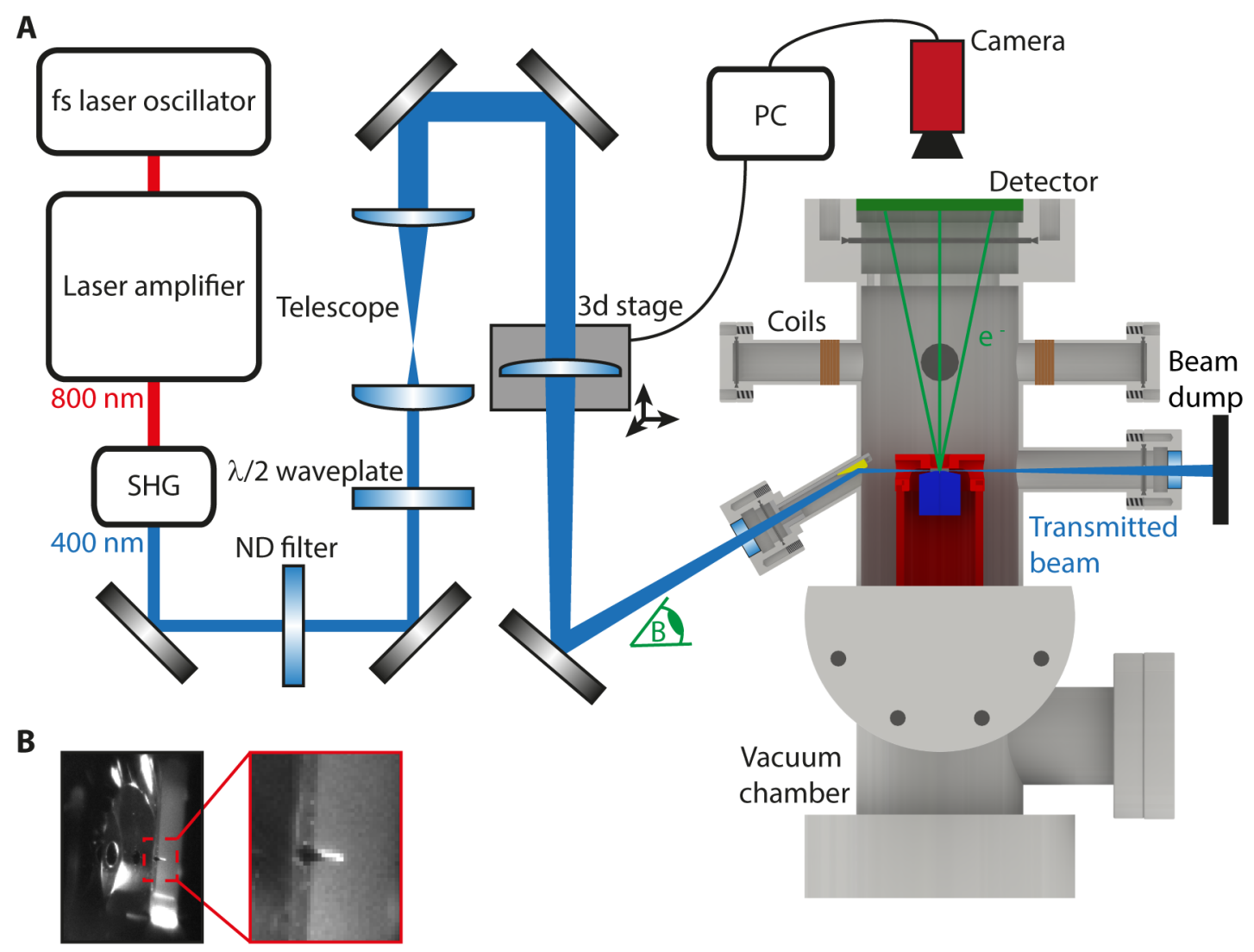

Figure 4.4: Experimental setup. A: Schematic of the laser beam setup and optical components. Pictures are not to scale. B: View onto the suppressor electrode and tungsten tip from outside the chamber via the implemented mirror. C: Electron gun, see Fig. 4.1.

chamber using only the gun assembly. The chamber is built in an identical way as the respective part of the TEM. The experimental setup is schematically shown in Fig. 4.4.

Laser pulses (red and blue lines) for photoemission of electrons (lime green) are generated by a Coherent Vitara Ti:sapphire oscillator ${ }^{1}$ seeding a Coherent RegA 9040 regenerative amplifier (800 nm center wavelength, $250 \mathrm{kHz}$ repetition rate $^{2}, 6 \mu \mathrm{J}$ pulse energy, pulse duration $<40 \mathrm{fs}$ ). Subsequently, a $\mathrm{BBO}^{3}$ crystal is utilized to frequency-double the laser light (second harmonic generation, SHG) ${ }^{4}$. The generated $400 \mathrm{~nm}$ laser pulses are used to generate electrons from the employed tungsten tip by nonlinear photoemission. Beforehand, the pulse energy is reduced to $4 \mathrm{~nJ}$ (except for the measurement of the pulse energy dependency) using a neutral density (ND) filter. The polarization state of the

\footnotetext{
${ }^{1}$ Center wavelength $800 \mathrm{~nm}$, pulse energy $6 \mathrm{~nJ}$, pulse duration $<20 \mathrm{fs}$.

${ }^{2}$ The laser system features a variable repetition rate (single shot to $800 \mathrm{kHz}$ ). However, for all experiments presented in this work, the repetition rate was set to $250 \mathrm{kHz}$.

${ }^{3}$ Beta barium borate; BBO is a common crystal used in nonlinear optics to, among other things, generate second harmonic radiation. For more information about frequency doubling see e.g. Ref. [78].

${ }^{4}$ The BBO crystal is part of the laser system's optical parametric amplifier (Coherent OPA 9450).
} 
linearly polarized laser light is adjusted by a $\lambda / 2$ wave-plate, and, unless stated otherwise, the polarization is set parallel to the tip axis. The beam diameter is increased to about $10 \mathrm{~mm}$ by the use of two plano-convex BK7 glass lenses in a telescope.

A third lens (focal length $300 \mathrm{~mm}$, BK7 glass) focuses the laser beam onto the tip emitter. To be able to move the focused laser spot, this lens is mounted onto a 3-dimensional translation stage. The stage can be manually moved by $25 \mathrm{~mm}$ along the propagation direction, and motorized in the two transverse directions utilizing a Newport OpticallyEncoded Translation Stage ( $80 \mathrm{~nm}$ resolution, $25.4 \mathrm{~mm}$ travel range) in combination with a Newport Picomotor Actuator (25.4 mm range, $<30 \mathrm{~nm}$ step size) and a Closed-Loop Controller.

With the movable lens, the laser is focused into an ultra-high vacuum chamber. This custom-made chamber is evacuated to a pressure on the order of $10^{-9}$ mbar by a serial connection of a scroll pump and a turbo-molecular pump. The low pressure ensures that the emitter remains free from contamination and electrons are not scattered on the path to the detector. It is also built to resemble the characteristics of the TEM head ${ }^{5}$ of a JEOL JEM-2100-F microscope. Hence, the laser beam is coupled into the chamber through a sapphire window (1.5 mm thickness) via an angled pipe and a custom-built mirror (UV-enhanced aluminum coating) onto the emitter tip. Fig. 4.4B shows an image of the tip reflected by the mentioned mirror, which was taken with a camera from outside the chamber. It demonstrates the accessibility of the tungsten tip for the laser light.

A micro-channel-plate (MCP) with phosphorous screen (Hamamatsu F2223-21PGF) is used to detect emitted electrons in a distance $75 \mathrm{~mm}$ away from the tip. To slightly correct for a possible mismatch between the electron gun axis and the center of the detector, electromagnetic deflection coils are used (cf. Fig.4.4A). A distortion of the spatial distribution as a consequence was not found.

The detector screen is monitored by an Allied Vision Technologies Pike F-032B camera (if not stated otherwise). For some high-accuracy experiments, the camera model Zyla 5.5 sCMOS from Andor Technology is used.

\footnotetext{
${ }^{5}$ The topmost part of a TEM, composed of the TEM gun assembly (suppressor, tip emitter, extractor and focus electrode) and accelerating section is denoted TEM head.
} 

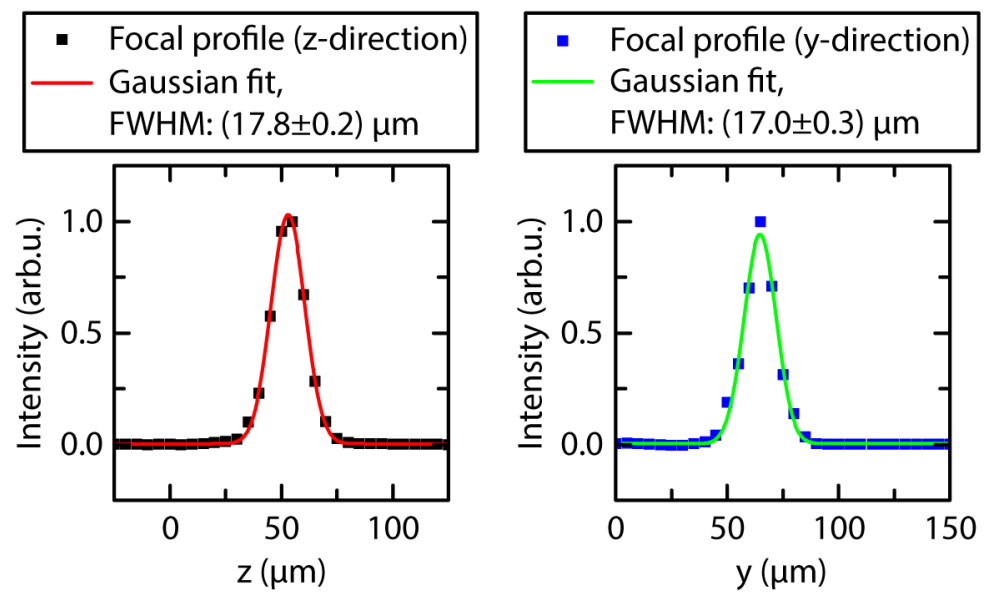

FiguRE 4.5: Laser spot size in two perpendicular directions. Along ( $z$-direction) and orthogonal ( $y$-direction) to the tip emitter axis.

A Labview ${ }^{6}$ program written specifically for these experiments controls the lens position, voltages at the emitter and suppressor electrode and saves pictures recorded by the camera to the hard drive of the computer.

\subsection{Laser focus size}

The laser focus size is important to calculate the laser intensity and to know the illuminated area on the tip emitter. The spot size is measured by the so-called knife edge method. Here, a gold film evaporated onto a sapphire substrate is used. The sharp transition between the gold and the pure sapphire allows for a measurement of the focus size by scanning the laser beam across this edge and measuring the transmitted laser power. The derivative of the obtained intensity scan yields the focus size along the scanned direction. For this measurement, the gold-sapphire glass was placed $300 \mathrm{~mm}$ behind the movable lens from the setup described in the previous section (the last mirror outside the vacuum chamber had to be removed). Laser power measurements are done by a Thorlabs Photodiode Power Sensor (model S120VC, used with Power Meter Console PM100D).

For the system used, a focus size of $18 \mu \mathrm{m} \times 17 \mu \mathrm{m}$ full-width-at-half-maximum (FWHM) is achieved (see Fig. 4.5), limited by the focal length of $30 \mathrm{~cm}$.

\footnotetext{
${ }^{6}$ National Instruments Labview is a programming environment for implementing measurement and control software.
} 
A

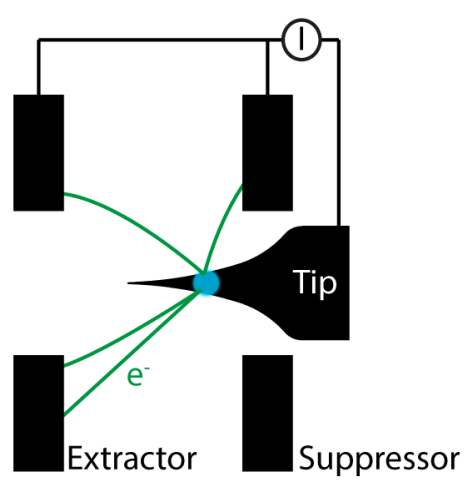

B

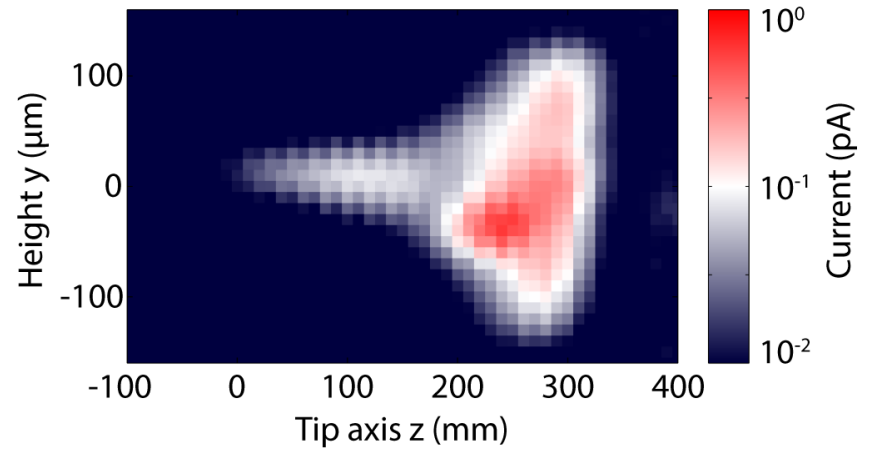

FiguRE 4.6: Measured current between tip and short-circuited electrodes in an $x y$-scan across the tungsten tip emitter. A: Sketch of the measurement principle. B: Measured $x y$-scan without applied voltages at the electrodes.

\subsection{Photocurrent measurement}

A challenging task after the assembly of the electron gun is to overlay the laser focus with the employed tungsten tip. If the focus electrode is not installed, the laser beam can propagate through the extractor electrode and through a second sapphire window flange (cf. Fig. 4.4A). In this case, the shadow image of the emitter is sufficient to find the overlap. However, in the case of an installed focus electrode, or later in the TEM itself, a different approach is needed. One viable way is to measure the electric current emitted by the tip.

Such measurements were carried out with a Keithley Electrometer (model 6514). The ability to measure currents below $1 \mathrm{pA}$, however, demands some restrictions. The maximum tip voltage which can be applied when measuring with such a device is rather small $(<40 \mathrm{~V})$. Therefore, no voltages were applied to the setup during this experiment. Furthermore, the expected current of nonlinear photoemission from a tungsten tip is well below $1 \mathrm{pA}$ in the region of the apex. Hence, to achieve maximum signal to noise ratio, all electrodes besides the tip where short-circuited.

The current map of this measurement for a scan of the laser spot across the emitter region is plotted in Fig. 4.6. The tip shape and an approximate location of the tip apex are clearly visible. It is not surprising that the signal stemming from the broad tip shaft is larger than that from the apex. Even with a field enhancement at the apex $(\beta \sim 5)$, for a two-photon process, the ratio between the electron yield of the apex and shaft region is estimated to be $N_{\text {apex }} / N_{\text {shaft }} \approx 0.04$. 
In conclusion, the electrical current measurement through the tip is a very useful way to determine the position of the laser focus relative to the emitter.

With a maximum current of $I_{\max }=0.6 \mathrm{pA}$, an estimation of the number of electrons emitted per laser pulse can be made. An upper limit is given by $I_{\max } /\left(e \cdot f_{\text {rep }} \approx\right.$ $15 e^{-} /$pulse), where e is the elementary charge and $f_{r e p}=250 \mathrm{kHz}$ the repetition rate of the laser system. Hence, the assumption of being below the space charge limit seems justified $([29,144]$.

\subsection{Nonlinearity of the photoemission process}

Before discussing the experimental findings regarding the spatial electron distribution of a laser-illuminated tungsten tip, the emission process should be addressed and clarified. The work function of the tungsten depends on the crystallographic surface orientation and varies between $4.2 \mathrm{eV}$ for the $\langle 310\rangle$ direction and $5.3 \mathrm{eV}$ in $\langle 110\rangle$ orientation [69]. For polycrystalline tungsten, a work function of $4.6 \mathrm{eV}$ is found when assuming a uniform distribution of crystal orientations [133]. With a photon energy of $3.1 \mathrm{eV}(400 \mathrm{~nm}$ wavelength), a two-photon photoemission process is expected. For confirmation, a measurement of the electron current as a function of incident laser power is shown in Fig. 4.7. In the low pulse-energy regime, the electron number within an image of the CCD-camera is obtained by a computer algorithm finding local maxima. For higher fluences, the image intensity is used to acquire the electron count rate. Since the image intensity depends linearly on the impinging number of electrons, this method holds as long as neither the detector nor the image recorded with the camera are saturated [29, 35, 144, 145]. For this purpose, the Zyla camera is used, as it provides a larger dynamic range.

A clear apparent slope of value two in a double-logarithmic plot confirms the two-photon process (cf. Eq. 2.9) [79-81]. A deviation from a two-photon process is not expected for these experimental settings, since the excess energy is large enough $(\sim 1.5 \mathrm{eV})$ to compensate for potential contaminations of the tungsten tip. This contamination can lead to an increase or decrease of the work function. Furthermore, cumulative heating effects can be neglected for a repetition rate of $250 \mathrm{kHz}$, as it results in a pulse to pulse interval of $4 \mu \mathrm{s}$, which is sufficient for the phonon system to equilibrate. The heating of the electron system due to the laser excitation on the other hand needs to 


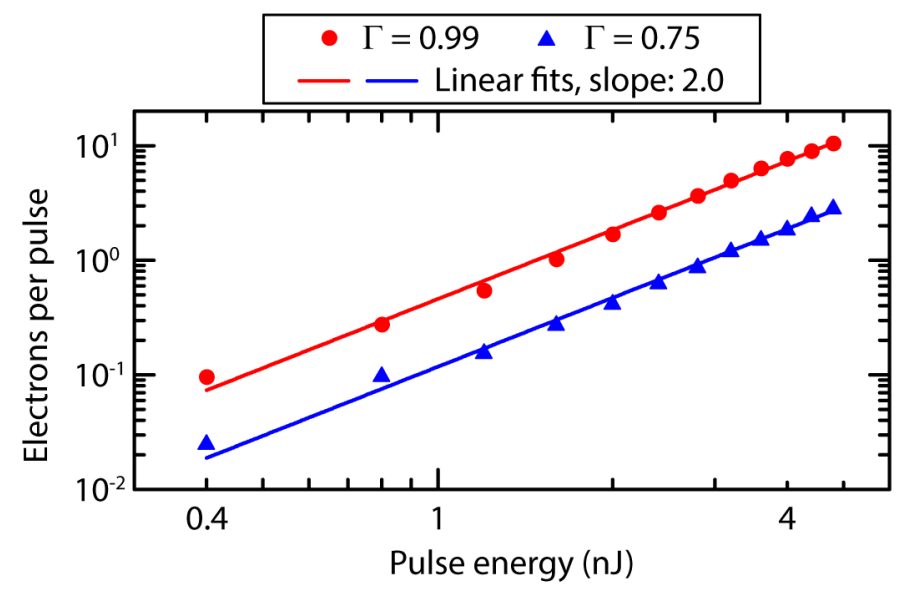

Figure 4.7: Electron yield as a function of the pulse energy for two different values of $\Gamma$. Curves are measured under illumination of the tip apex. Dots represent measurements, and lines correspond to a linear fit of the data (apparent fit for double-logarithmic scale).

be considered separately. For an intensity of $4 \cdot 10^{10} \mathrm{~W} / \mathrm{cm}^{2}(5 \mathrm{~nJ}$ pulse energy, $20 \mu \mathrm{m}$ FWHM focal size, 40 fs pulse duration) the temperature of the electron gas is increased to about $1500 \mathrm{~K}$ [146]. Therefore, thermally assisted photoemission is generally possible [81]. However, judging from the measured nonlinearity, it seems that this is not the dominant emission process in the present experiment. For a change in the nonlinearity due to effects regarding the ponderomotive energy, such as channel closing, a Keldysh parameter of one or below is necessary [29, 92, 145, 147]. However, even for the $5 \mathrm{~nJ}$ pulse energy in Fig. 4.7, only a Keldysh parameter of 31 is achieved. A reduction of the nonlinearity due to space charge effects can, apparently, also be ruled out with a maximum of 100 electrons per pulse $[26,29,144,148]$. Otherwise, the curve of the power dependency would saturate, as electron emission would be extenuated.

\subsection{Field emission}

In order to calculate the geometric factor $k$ for the electric field of a tip (see Sec. 2.2.3), and to check for the homogeneity of the detector, static field emission measurements are presented in this section. The calculation of $\mathrm{k}$ can be performed by employing the FowlerNordheim equation as shown below. Analyzing the homogeneity of the response of the detector is feasible when analyzing the emission patterns of more than one tip emitter. Static field emission offers the possibility to generate a large amount of electrons. Hence, a potential damaged detector regions are easily identified. Furthermore, by utilizing 

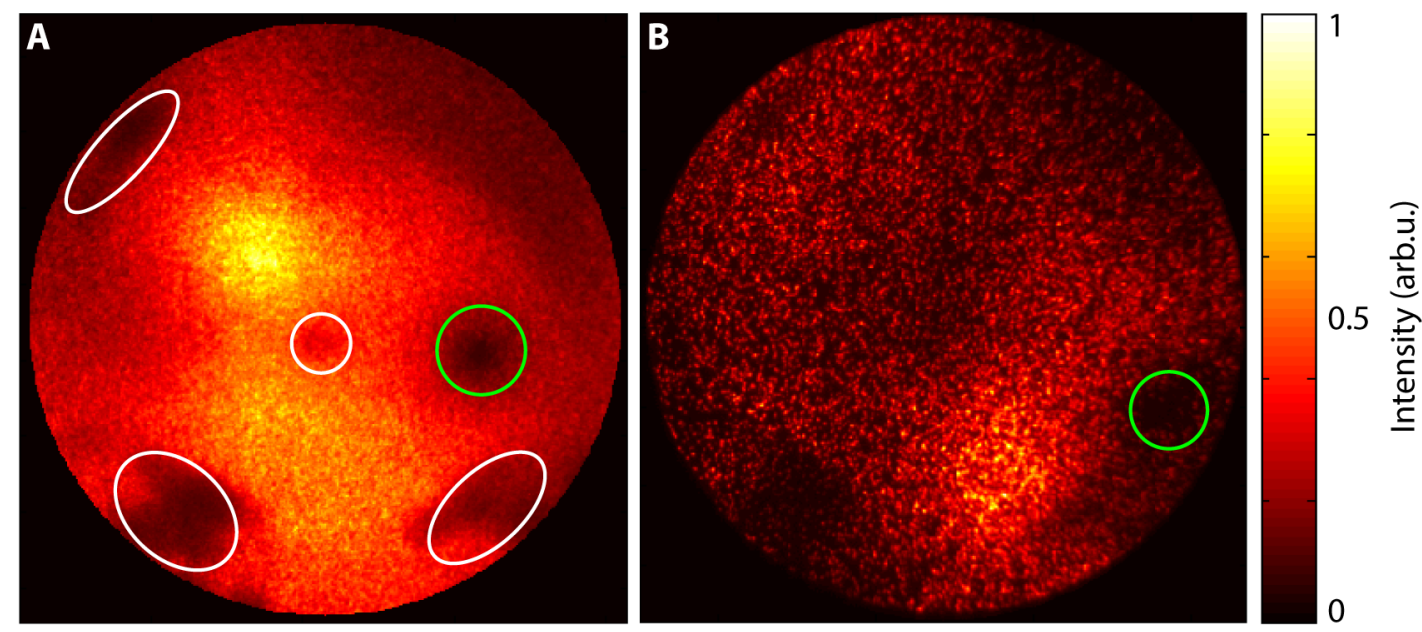

FIGURE 4.8: Field emission patterns for different employed tungsten tips. The intensity is individually normalized for each image. White ellipses mark areas of reduced gain on the detector. The region denoted by the green ellipse is due to a gain reduction caused by electrons with an incident angle similar to the detector channel angle.

more than one (polycrystalline) tungsten wire, chances are high that intensity maxima on the detector, which might occur due to the crystallographic orientation of the front facet, even out.

Fig. 4.8 shows field emission patterns on the detector. First, it should be noted, that the dark regions in Fig.4.8A (marked with white ellipses) originate from a weakened amplification of the detector. This is true also for the very center, which seems to be less bright than the surrounding signal. The green ellipse marks a region where electrons arrive on the detector with the same angle of MCP channels, resulting in less amplification. This spot shifts, e.g., if the magnetic coils around the chamber are used to apply a different magnetic field, or when a magnet is held next to the chamber, verifying this assumption. The position of the green circle also differs between Fig. 4.8A and Fig. 4.8B.

Within the field emission pattern, no direct identification of any facet of the tip was possible. This is to be expected since all employed tungsten tips, except one, were etched using a polycrystalline wire. Hence, even if some statistically distributed facets were to emit more electrons, a distinction is not possible.

A voltage-dependent measurement of the field emission current can be used to gain information about the work function and the field enhancement factor $k$. For this purpose, a so-called Fowler-Nordheim plot is used, where the ratio $J / F^{2}$ is plotted versus $1 / F$, with $J$ being the current density and $\mathrm{F}$ the electric field at the tip. Since the electric 
field $F$ cannot be directly measured, instead, the applied voltage $U$ is used in the plot, utilizing the relation $F=U /(k r)$ [67]. For calculating the current density, the electron number was divided by the camera integration time and the emission area $A_{\text {em }}$. Multiplying with the elementary charge yields the current density. Assuming an emission from the apex area, which can be approximated as a half sphere, and further presuming that only half of this area contributes, since the tunnel current is field dependent and thus maximal in forward direction, the emission area can be defined as $A_{e m}=\pi r_{\text {tip }}^{2}$. The number of electrons in a detector image was counted for low numbers of electrons with a counting algorithm. This also yields an average intensity for one electron on the detector. Thus, for higher numbers the total intensity of the detector, weighted with the intensity of a single electron, is used to compute the electron count rate. The Fowler-Nordheim equation is written

$$
J=A \frac{(\beta U)^{2}}{\Phi} \exp \left(\frac{B \Phi^{3 / 2}}{\beta U}\right)
$$

with $A=\frac{e^{3}}{16 \pi^{2} \hbar}, B=-\frac{4 \sqrt{2 m_{e}}}{3 \hbar e}$ and $\beta=\frac{1}{k r}$, where $k$ is the geometric correction factor and $\mathrm{d}$ the tip apex diameter. In the Fowler-Nordheim plot, the measurements of a field emission curve will result in a straight line, which is then fitted. The slope $\xi$ and intercept $\eta$ of the fit yield values for the work function $\Phi$ and the field enhancement factor $\beta$ :

$$
\begin{gathered}
\Phi=\frac{\xi \sqrt{\frac{\exp (\eta)}{A}}}{B}, \\
\beta=\sqrt{\frac{\Phi \exp (\eta)}{A}} .
\end{gathered}
$$

Unfortunately, fitting experimental data using the intersection of the linear fit for calculation tends to result in unrealistic values. One reason is that the exact current density would be required and the value of the gained intersection is very sensitive to these numbers. Therefore, usually only the slope is used. In order to calculate either the work function or the field enhancement factor, an assumption on the respective other quantity is made.

Fig. 4.9 shows a Fowler-Nordheim plot from a tungsten tip. Since the work function $\Phi$ is more properly known than the enhancement factor $k$, here, only $k$ will be calculated. By assuming a work function of $\Phi=(4.6 \pm 0.5) \mathrm{eV}$ (calculated with values taken from 


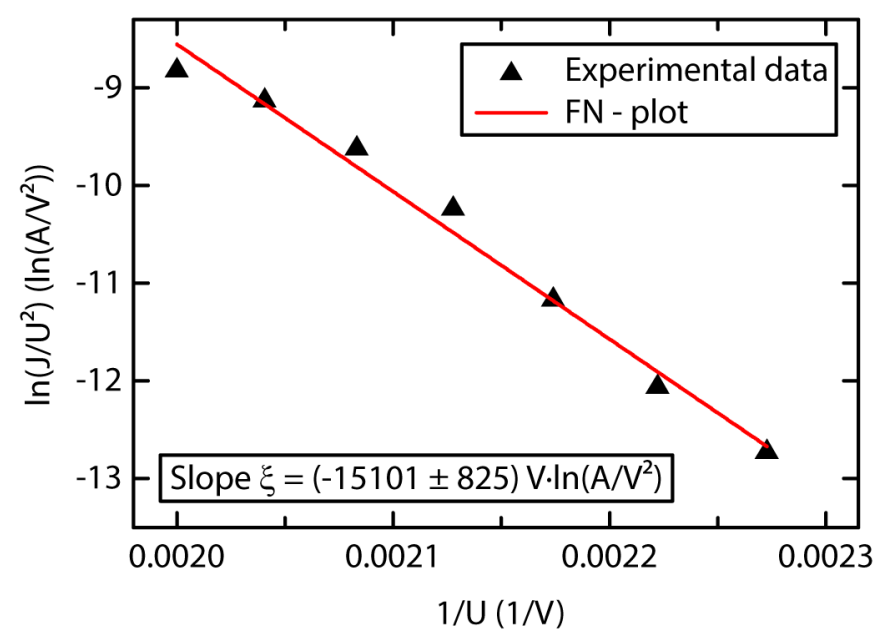

FiguRE 4.9: Fowler-Nordheim plot acquired from a tungsten tip within the gun assembly.

Refs. $[69,149,150])$, the field enhancement factor and $k$-parameter can be calculated from the slope $\xi$ of the linear fit using

$$
k=\frac{\xi}{B \cdot \Phi^{3 / 2} \cdot r_{t i p}^{2}},
$$

which results in a value of $k=2.2 \pm 0.4$. Compared to the literature, this value appears to be small (cf. Sec. 2.2.3). However, aside from the precise tip geometry, the presence of the suppressor electrode has a strong impact on the exact electric field as well, which is why the calculated value differs from the typical value found for a tip opposing an anode. The empirical formula

$$
k=0.59 \cdot \vartheta^{1 / 3} \cdot(x / r)^{0.13}
$$

describes the calculation of $k$ as a relation between the tip radius $r$, the tip-to-screen distance $x$, the emitter-cone half angle $\vartheta$ (in degree) [67]. For the employed gun assembly $\left(r=(100 \pm 20) \mathrm{nm}, x=(350 \pm 20) \mu \mathrm{m}, \vartheta=(5 \pm 1)^{\circ}\right)$, the above stated formula yields a value of $k=2.9 \pm 0.2$, which agrees well with the above calculated value from the measurement. 


\section{Chapter 5}

\section{Spatial distribution of photoemitted electrons}

In order to successfully align an ultrafast TEM with regard to beam brightness, emittance and pulse duration, knowledge about the emission site of the emitted electrons is important. To this end, the electron emission pattern from tungsten tips upon femtosecond laser irradiation are investigated in this chapter. First, simulated electron trajectories are introduced. Subsequently, experimental results are shown and compared to the results obtained with the simulations. Under illumination of different regions on the tip emitter, conclusions regarding the emission site can be drawn.

\subsection{Electron trajectories}

Successfully understanding the spatial electron distribution requires knowledge about the electron trajectories. To this end, calculated electron trajectories are shown in this section. The computation is performed for the test chamber as described in Fig. 3.1 (see Fig. 4.4 for setup) and the associated section.

Throughout this work, a distinction is made between two emission sites: emission from the tip apex and emission from the tip shaft. The tip apex refers to the front-most part of the tip emitter and spans the region where the shape of the tip resembles a sphere (cf. Fig. 5.1). The surface beyond the apex radius does not belong to the apex region anymore, but to the tip shaft. 


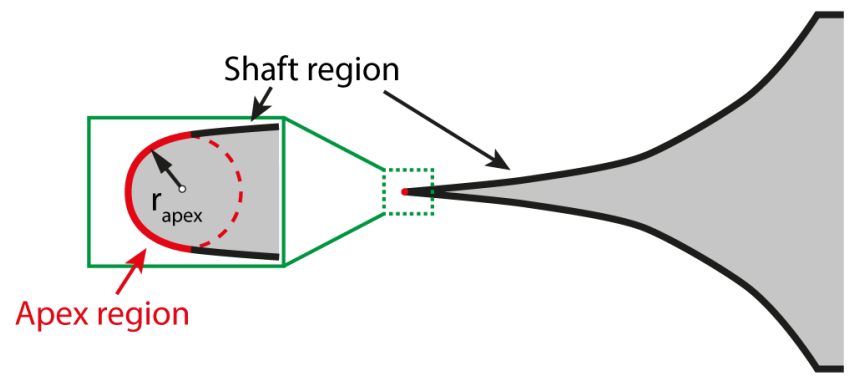

FIGURE 5.1: Sketch of the tip emitter indicating shaft region (black) and apex region (red).

Simulated trajectories are plotted in Fig. 5.2 for different voltage ratios $\Gamma$ (see Eq. 3.7 for definition) and in Fig. 5.3 for different emission angles. In both pictures, trajectories are computed with an initial kinetic energy of $1 \mathrm{eV}$. Orange lines depict trajectories stemming from the apex, green lines those from the tip shaft. In addition, dark green marks trajectories which impinge at the detection plane, whereas light green lines depicts those electrons which are blocked by the extractor. For every setting, four different magnifications (I-IV) are shown to facilitate reading the description of the electron trajectories. To be able to identify trajectories which cross the symmetry axis, only curves from one side of the emitter are presented.

In Fig. 5.2, different voltage ratios are applied and electrons are emitted perpendicularly with respect to the tip surface. The cutoff point (electric force free point, cf. Sec. 3.2) is shifted towards the apex from Fig. 5.2A to Fig. 5.2C. Electrons emitted with an angle $\left( \pm 90^{\circ}\right)$ towards the surface normal are shown for a fixed voltage ratio in Fig. 5.3.

Considering apex electrons (orange trajectories), an increasing $\Gamma$ parameter leads to a more focused pattern on the detection plane. Furthermore, more electrons are able to pass the extractor aperture, and hence, the overall electron yield (on the detector) increases. For electron emission normal to the tip surface, apex electrons can be detected in a region which is set by the geometric opening angle between the tip apex and the extractor aperture. This statement remains valid unless the electrons are focused more tightly, which occurs for $\Gamma$ values close to the field reversal point, as visible in Fig. 5.2C. Assuming electron emission from every side of the tip apex, a homogeneous circular area pattern is expected at the detector. This assumption, however, is neither taking into account the size of the emission area (cf. Fig. 3.2 and Eq. 3.4), nor the changing field enhancement along the tip surface (cf. field distribution in Fig. 2.5). The emission 
A

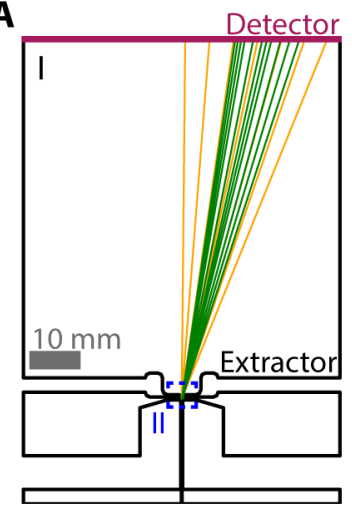

B
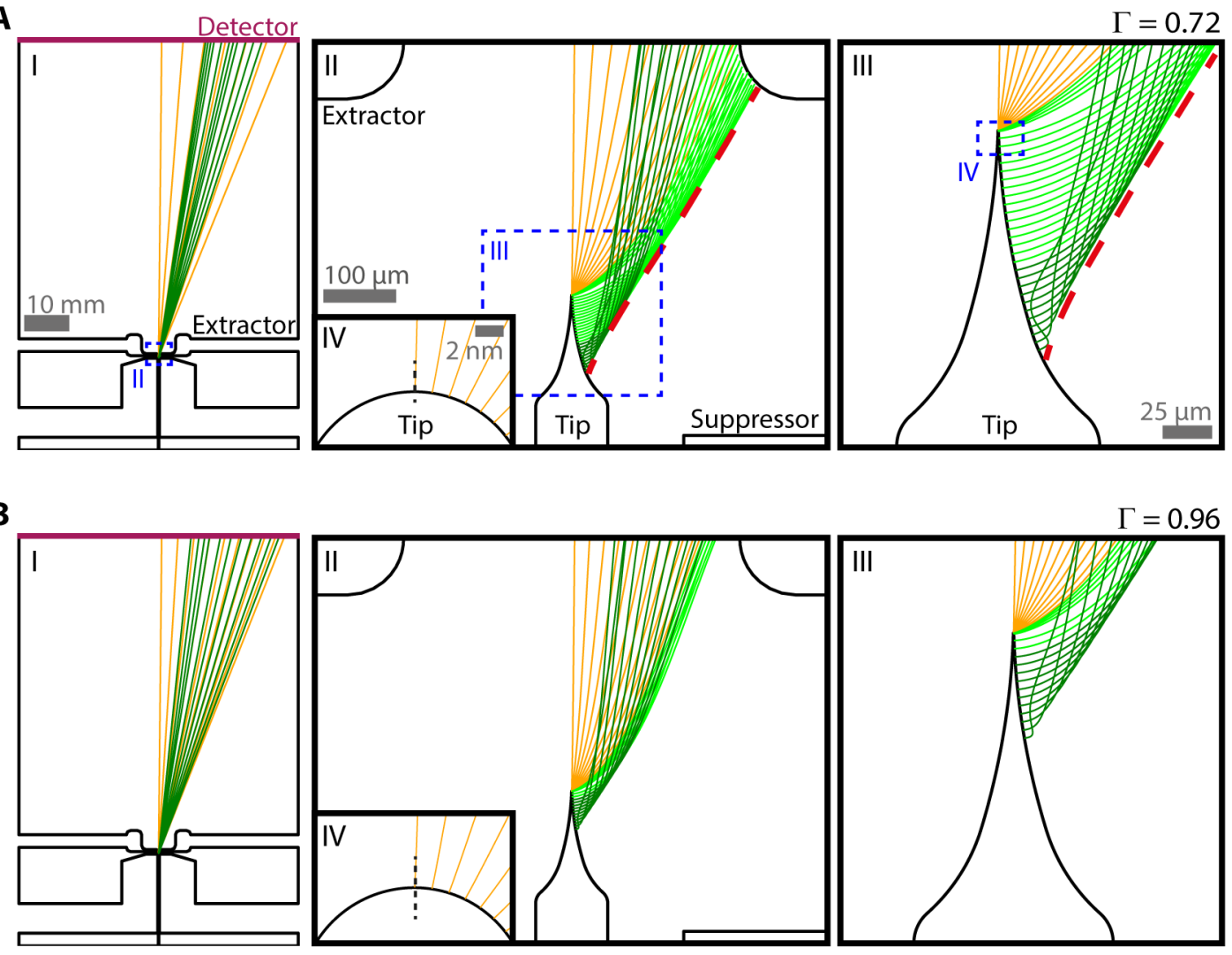

C
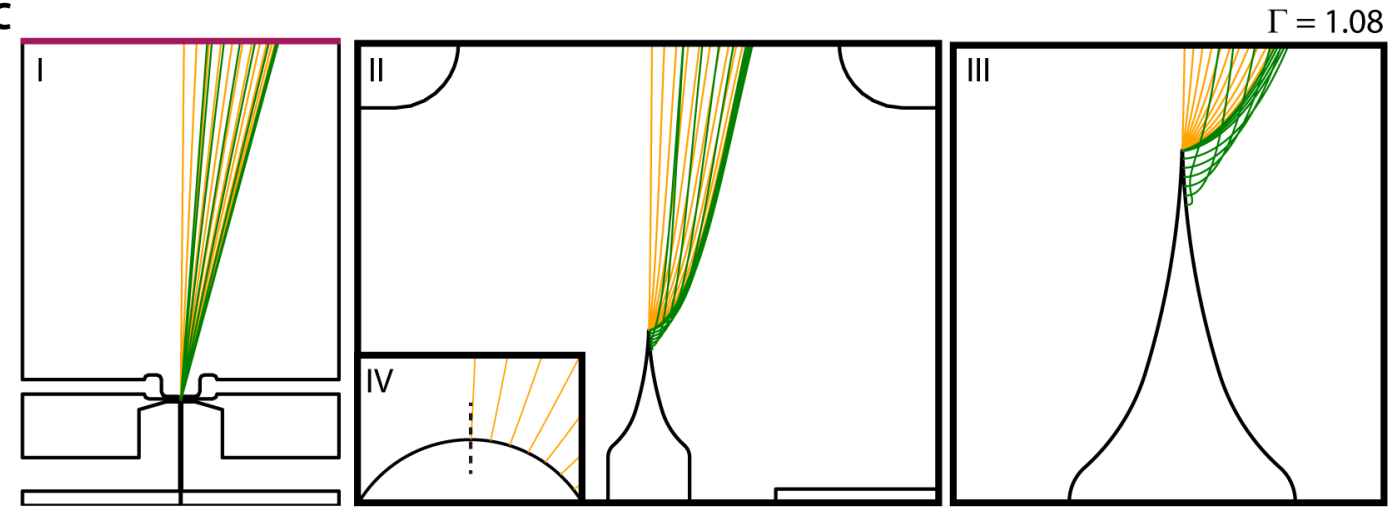

FIgURE 5.2: Exemplary electron trajectories for three different voltage settings (A: $\Gamma=0.72, \mathrm{~B}: \Gamma=0.96, \mathrm{C}: \Gamma=1.08)$. Four magnifications are shown, where subfigures I show the overall simulated geometry. Orange lines correspond to trajectories stemming from the apex, and green lines to those originating from the tip shaft. Light green incicates electrons blocked by the extractor, whereas dark green shows trajectories which reach the detector (red in subfigure I). The dashed line in subfigures IV indicate the surface normal. 
pattern remains homogeneous only if the increasing emission area compensates for the decreasing field enhancement.

A more complex picture is given for emission from the shaft site of the emitter. For each voltage setting, the so-called cutoff-point marks the transition of the electric force pointing away from the tip emitter to pointing towards the tip emitter. The location of this point therefore depends on the applied voltages shifting towards the tip apex for increasing values of $\Gamma$ (cf. Sec. 3.2). Electron emission is generally possible from the apex up to this cutoff-point. An electron emitted in the vicinity of the cutoff-point experiences a stronger acceleration in the $z$-direction ${ }^{1}$ than electrons emitted further away from this point. Furthermore, due to the orientation of the shaft surface, an electric force in $r$ direction (for coordinates see Fig. 3.1) affects the electron trajectories. This leads to a strong bending of electrons emitted close to the cutoff point and results in trajectories that pass the extractor aperture (see e.g. green lines in Fig. 5.2A-III). The strength of this bending decreases with increasing distance from the cutoff point. In Fig. 5.2, a line can be identified which appears to act like a "mirror line", i.e. electron trajectories seem to be reflected at this line (shown as red dashed line in Fig. 5.2A(II,III)). Hence, the "inner electrons" on the detector (closest to the center of the pattern) stem from the part of the shaft which is closest to the cutoff point and farthest away from the apex. Since the cutoff-point shifts towards the apex with increasing $\Gamma$, the region on the tip shaft from, where electrons are able to reach the detector shifts as well.

Upon illumination of the tip emitter with a laser pulse, the pattern on the detector depends also on the laser focus size, which leads to a "selection" of electron trajectories. A shift of the laser focus position towards the tip apex at fixed voltage settings would result in an increasing pattern diameter with increasing dark area in the center. The reason for this effect is, that electron trajectories originating from a region closer to the apex impinge at larger radii from the center. If the cutoff point is close to the apex region, the trajectories stemming from the apex are bend in the same way as the shaft electrons. Therefore, in this regime a clear distinction between shaft and apex electron is impossible at the detection plane.

Fig. 5.3 shows a comparison of different emission angles $\alpha$ with respect to the surface normal. For this non-perpendicular emission, the patterns gained from apex electrons

\footnotetext{
${ }^{1}$ The $z$-direction is the direction along the tip axis. Here, cylindrical coordinates are used $(r, \varphi, z)$.
} 

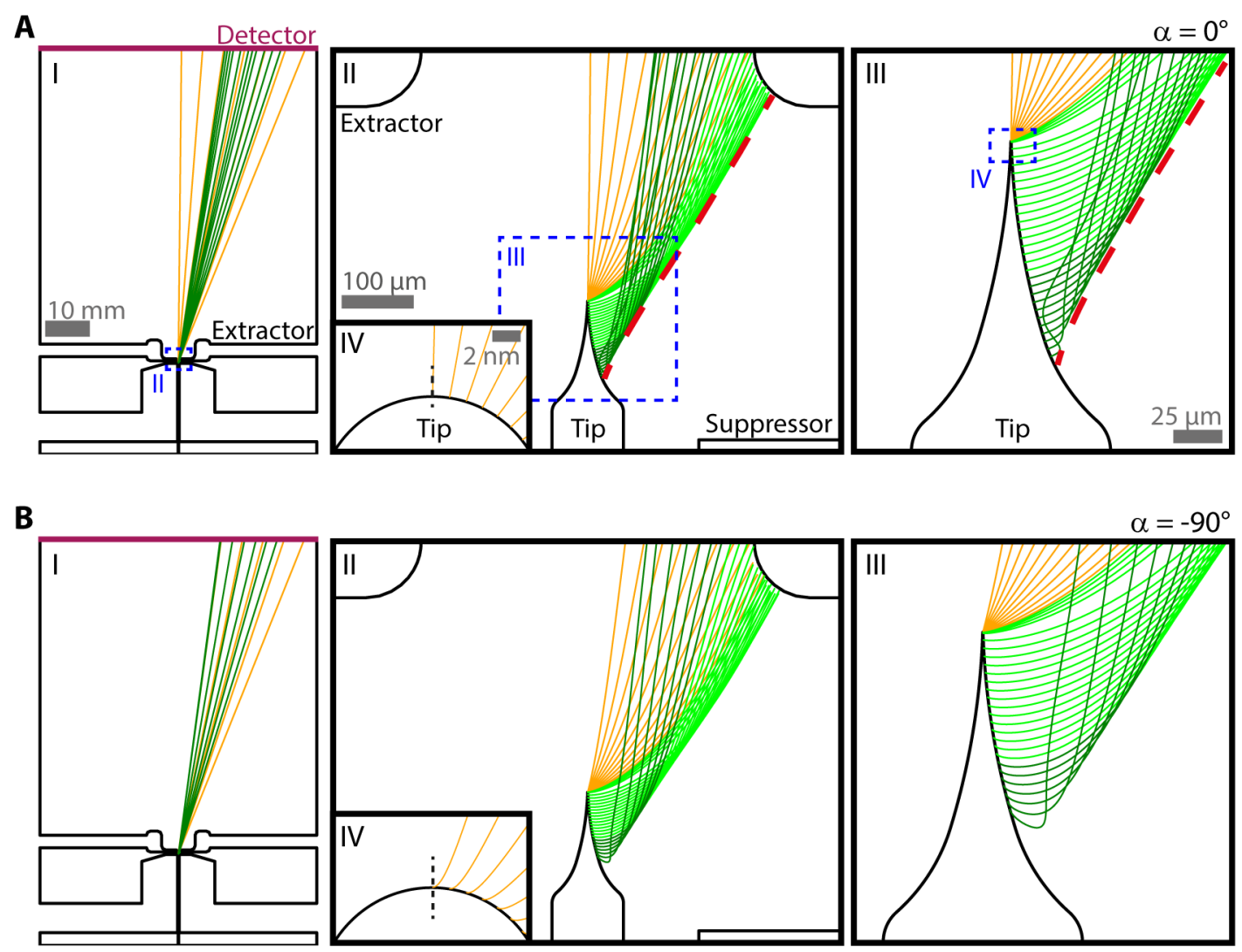

C
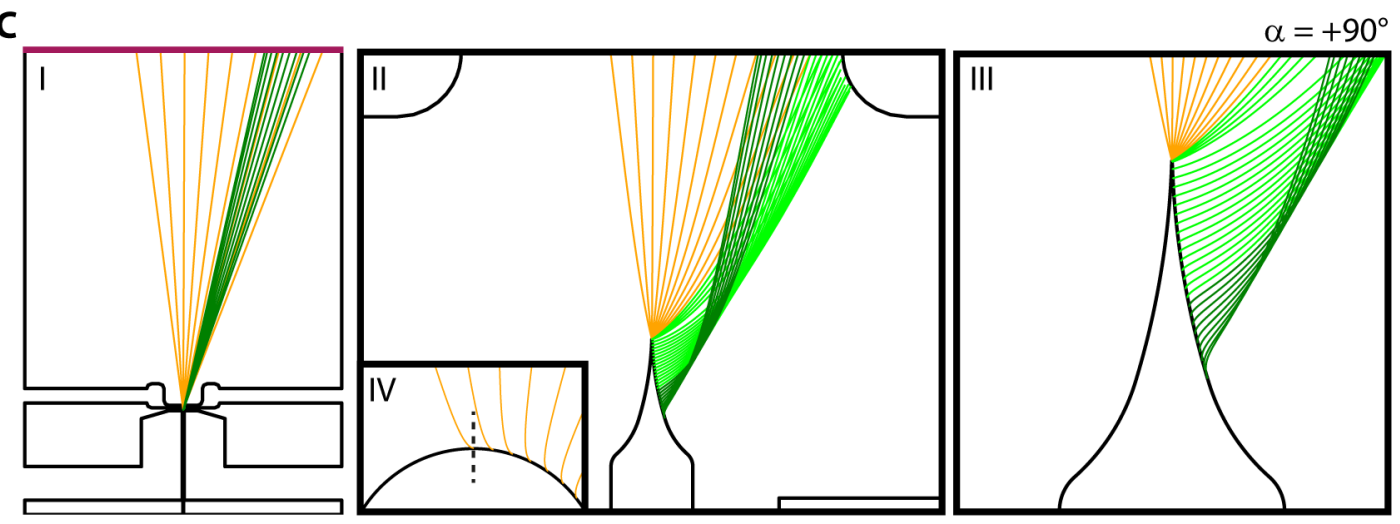

FiguRE 5.3: Electron trajectories for different emission angles $\alpha$ with respect to the tip surface for a fixed voltage setting $\left(\mathrm{A}: 0^{\circ}, \mathrm{B}:-90^{\circ}, \mathrm{C}: 90^{\circ}\right)$. Likewise to Fig. 5.2, different zoom levels are shown, electron trajectories are color coded and the dashed line in subfigures IV indicate the surface normal to emphasize the emission angle. 
result in similar figures as for the emission along the surface normal. For emission angles $-90^{\circ}$ to the surface normal, i.e., away from the symmetry axis, more electrons are blocked by the extractor aperture. The pattern on the detector screen has an inner area where no electron trajectories end. For emission in $90^{\circ}$ direction, more electrons reach the detection plane. Some trajectories cross the symmetry axis, which is important for later discussion of symmetric patterns upon asymmetric illumination. In a simplified description, one can consider electrons emitted with an angle to the surface normal as electrons emitted perpendicularly to the surface but with a shifted starting point on the tip surface. However, the effect of this "shifted starting position" depends on the initial kinetic energy, i.e. for a low initial energy the effect is smaller than for large initial energies.

Electron trajectories from the shaft follow the same pattern. Hence, emission with an angle $\alpha=-90^{\circ}$ results in trajectories which end closer to the symmetry axis and emission in $90^{\circ}$ direction leads to a larger dark area in the middle (considering only shaft emission).

In order to simulate laser induced electron emission patterns, the calculated trajectories are weighted according to the probability density function described in Sec.3.1.1. Calculated emission patterns are shown in the following section, together with measured images.

\subsection{Emission patterns (detector images)}

In this section, photoemission characteristics of a tungsten needle emitter within a suppressor-extractor geometry are studied. Experiments are conducted for different voltages applied to the gun assembly electrodes as well as for different positions of the laser focus relative to the tip emitter. In order to correctly describe the measured emission patterns, knowledge about the position of the tip apex is required. A method of acquiring the emitter position in general, and a coarse estimation of the tip apex position, is described in Sec. 4.5. A quantitatively more accurate method is to use a scan of the voltage ratio versus the laser focus along the tip axis as described later in Sec. 5.3. This part deals with the emission patterns received at different positions for various voltage ratios $\Gamma$ (see Sec. 3.2 for definition). 


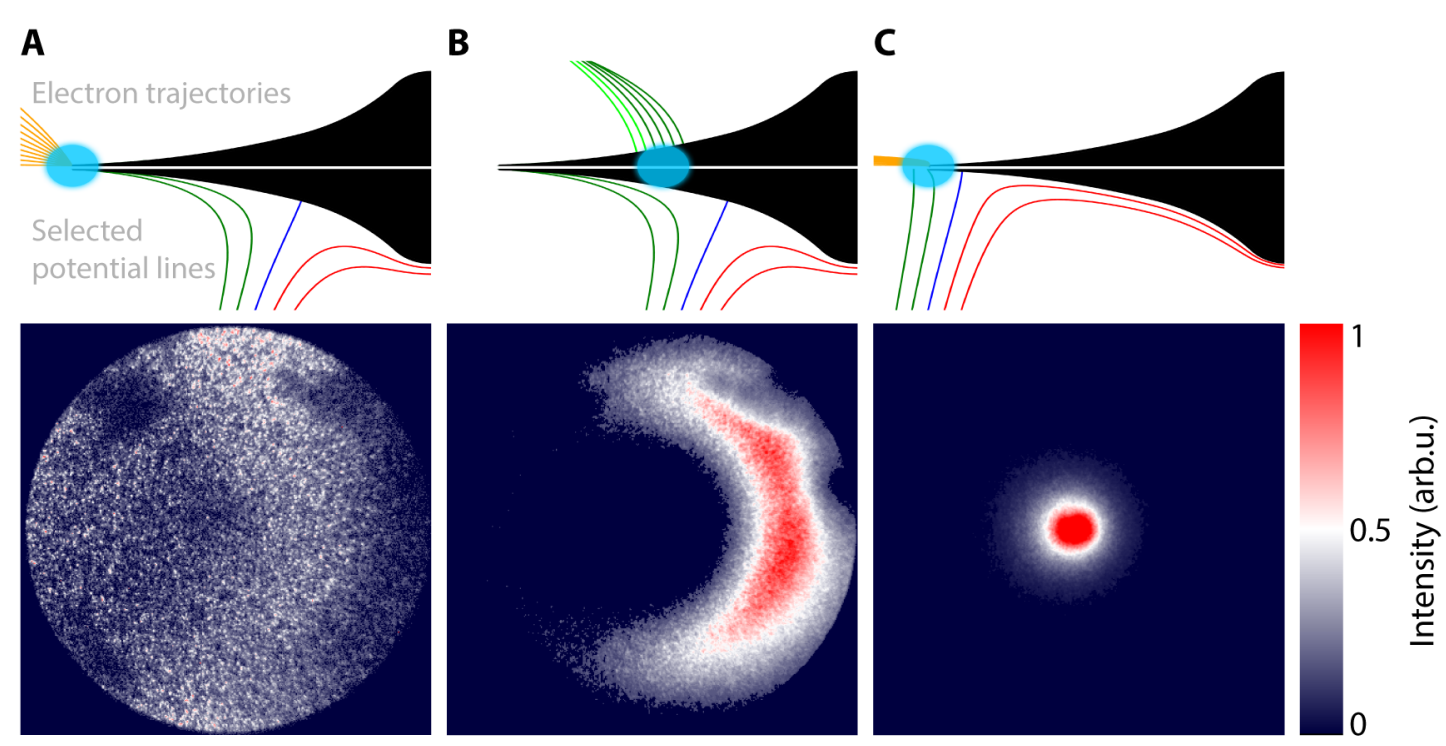

FiguRE 5.4: Three typical patterns (lower images) for different illumination and voltage conditions (sketched in upper images). Sketches depict the laser position (blue dot), electron trajectories (upper half of the sketch; orange and green lines similar to Fig. 5.2) and selected potential lines (lower half of the sketch; green, blue and red lines similar to Fig. 3.3). A: Illumination of the tip apex and low voltage ratio $\Gamma$. B: Shaft illumination and $\mathrm{C}$ : High $\Gamma$ values and illumination of the tip apex.

As a function of the voltage ratio $\Gamma$ and the laser focus position, three typical patterns can be observed, as shown in Fig. 5.4. The first pattern shows a homogeneous distribution throughout the detector area, which indicates emission from the apex region. Fig. 5.4B depicts an arc-shape usually detected upon illumination of the shaft region and the last image shows a focused spot indicating that the voltage ratio $\Gamma$ is close to the field reversal point.

The desired pattern for ultrafast electron microscopy is, at this point, a homogeneous distribution of electrons within a confined ring in order to obtain maximum transmission through subsequent apertures. Furthermore, electrons within the electron beam should all emanate from the tip apex and not the shaft in order to receive a small (virtual) source size and a short pulse duration.

\subsubsection{Apex electrons}

The most anticipated emission pattern for laser induced electron emission from a needle emitter is a homogeneous distribution on the detector. However, this image will only form for electrons emitted from the tip apex at a voltage ratio $\Gamma$ significantly below the field reversal $(\Gamma \ll 1.2)$. For voltages close to the field reversal, a ring shape is observed, 


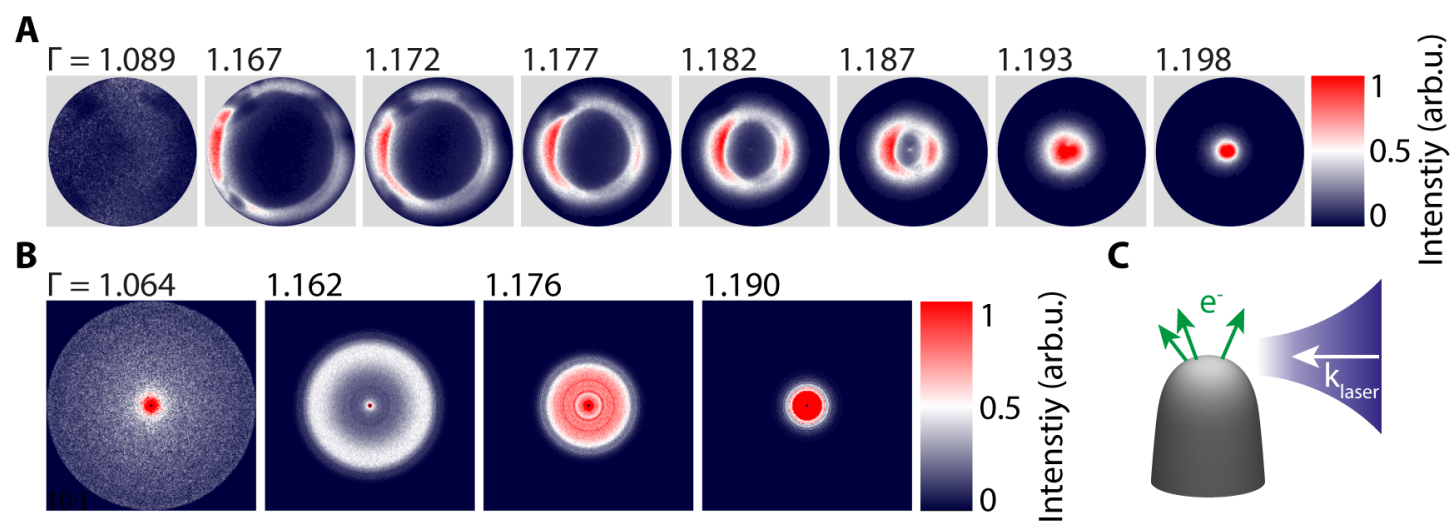

FiguRE 5.5: Emission patterns for different values of $\Gamma$ for illumination of the tip apex $(d=40 \mathrm{~nm})$. A: Measured data. B: Simulation results. The intensity is normalized to the same value across all images in $\mathrm{A}$ and $\mathrm{B}$, respectively. Only the first image $(\Gamma=1.064)$ in $\mathrm{B}$ is plotted with a ten times higher intensity. C: illustration of the direction of the incident laser light.

as depicted in Fig. 5.5A. By increasing the voltage ratio $\Gamma$, the ring shape is more and more focused, until it forms a spot and subsequently vanishes. If $\Gamma$ is decreased, the ring disappears from the detector, and a homogeneous pattern is observed.

This ring pattern solely occurs under illumination of the apex region and is only observed for an apex diameter below $100 \mathrm{~nm}$ (for $400 \mathrm{~nm}$ laser wavelength). For blunter tips, an arc shape is found, similar to illumination of the tip shaft (Fig. 5.4B). It seems that the geometry is too large to enable diffraction of the laser light around the tip with significant intensity, which restricts the electron emission to the illuminated side, resulting in an arc shaped emission pattern. However, a homogeneous emission pattern from the apex for low $\Gamma$-values (image for $\Gamma=1.089$ in Fig. 5.5A) is found for blunt as well. The homogeneous pattern across the whole detector implies, that diffraction around the apex itself is apparently significant, otherwise the (homogeneous) pattern should appear only on one side of the detector. To pursue this thought, a ring pattern for blunt tips might be present, as well. But only if the cutoff-point is very close to the apex, so that the arc (or ring) pattern stems from a region closer to the apex (cf. 5.2.4). In this scenario, the ring pattern is not observable with this experimental setup.

Another feature visible in Fig. 5.5A is the higher intensity of the ring on the left side, even though the laser illuminates the tip from the right side (cf. Fig. 5.5C). Similar observations have been made by Yanagisawa et al., who investigated the emission facet under laser illumination of sharp crystalline tungsten tips [30, 139, 141, 151]. They attributed the asymmetric pattern to an asymmetric field distribution over the tip apex 


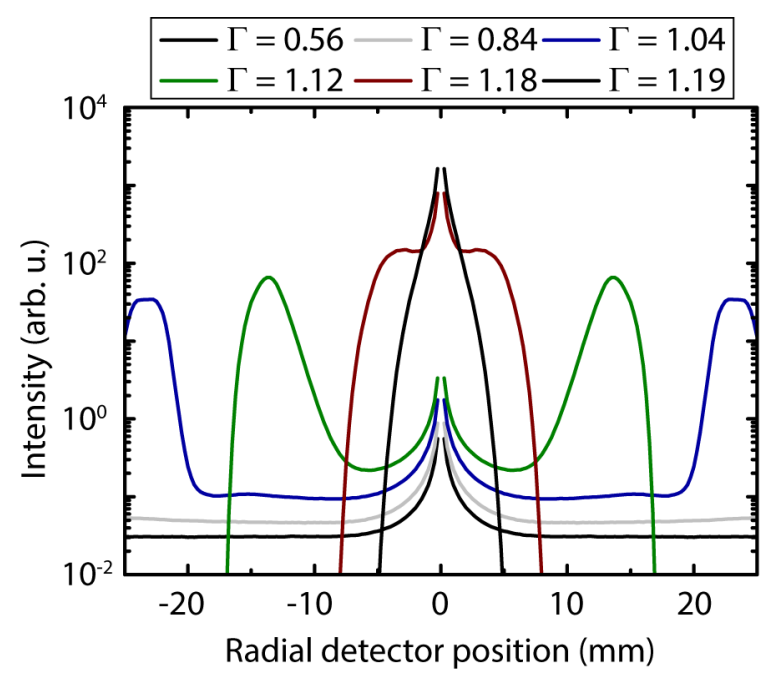

Figure 5.6: Cross section of simulated detector images for different values of $\Gamma$ and for illumination of the tip apex, assuming radially symmetric electron emission.

stemming from interference patterns of (photo-) excited surface electromagnetic waves (e.g. surface plasmon-polaritons, Zenneck waves [30, 152, 153]).

Detector patterns gained from the simulated data are shown in Fig. 5.5B. Since the simulation is done with cylindrical symmetry the information of the arrival points only exists along the radial component. The images are obtained by plotting radial arrival positions of electrons with a random azimuthal angle. To this end, $10^{6}$ electrons with different starting parameters are randomly chosen. A good agreement between the simulated and measured patterns is visible concerning the non-central area. In the center of the simulated patterns, a peak is observed, stemming from an effective $1 / r$ scaling. Whereas the very center of the pattern is expected to be an artifact, a central peak in intensity might stem from a caustic-like focusing effect of electron trajectories, since in Fig. 5.4B, the image for $\Gamma=1.187$ contains such a central feature, as well. For better visibility of the simulation results, the radial intensity distribution is again plotted in Fig. 5.6. Clearly visible are the peaks corresponding to the dominant ring pattern, which shift with increasing $\Gamma$ towards the center. The background signal between peaks is attributed to electrons emitted from the apex.

\subsubsection{Shaft electrons}

In Fig. 5.7, detector images from shaft-emitted electrons are shown. The intensity is again normalized for each image series in $\mathrm{A}$ and $\mathrm{B}$, respectively. 
A

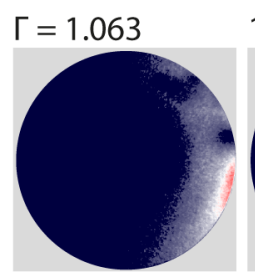

B

$\Gamma=1.036$

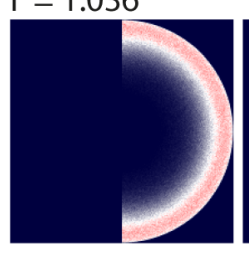

1.076

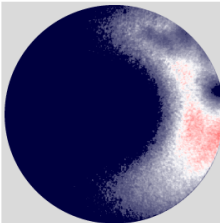

1.089

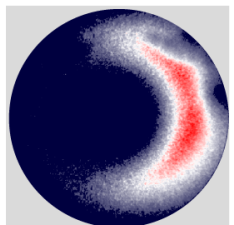

1.102

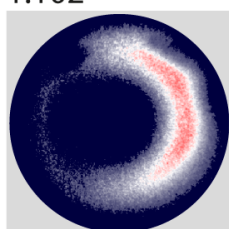

1.115

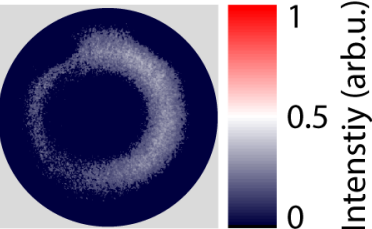

C
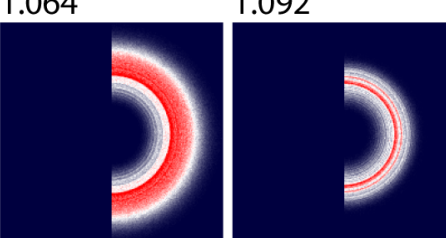

1.120
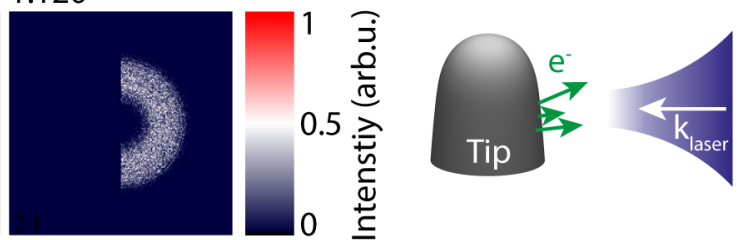

Figure 5.7: A: Detector images of electrons from the shaft region. B: Simulated emission patterns. The intensity is normalized across the pictures in A and B, respectively.

C: illustration of the direction of the incident laser light.

Similar to the emission from the apex region, a focusing effect occurs for increasing values of $\Gamma$. The main difference and hence the identifying feature of emission from the shaft occurs for large values of $\Gamma$. Before the electron emission is completely suppressed, the pattern incorporates a dark area in the center. This dark area is visible in the measurements as well as the simulated patterns (cf. image for $\Gamma=1.15$ in Fig. 5.7A and $\Gamma=1.120$ in Fig. 5.7B). Note that the intensity of the image for $\Gamma=1.120$ Fig. 5.7B is doubled compared to the other patterns. In comparison to the image for $\Gamma=1.092$, this illustrates the vanishing of the electron yield incorporating an electron-free center and a, compared to the apex electrons, large minimal pattern (cf. pattern sizes in Fig. 5.7 and Fig. 5.5).

This minimal arc is due to the radial starting position of the trajectory closest to the cutoff point. For illumination of the same shaft region and a voltage ratio close to emission cutoff, a ring pattern can form due to diffraction around the tip shaft (pattern for $\Gamma=1.115$ in Fig. 5.7). The effect becomes visible since the total number of electrons is very low.

For an excitation with increasing distance from the tip apex, the diameter of the dark area in the center of the pattern increases. This follows from the increasing radial distance of the shaft surface from the central symmetry axis. As outlined in the previous section, the radially innermost electrons originate from the area farthest away from the tip apex. The increase of the region in the center, where no electrons arrive on the 
A

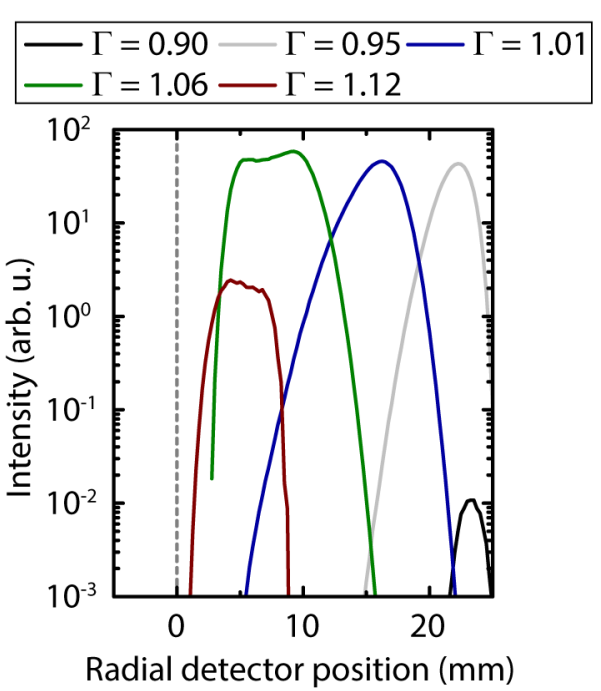

B

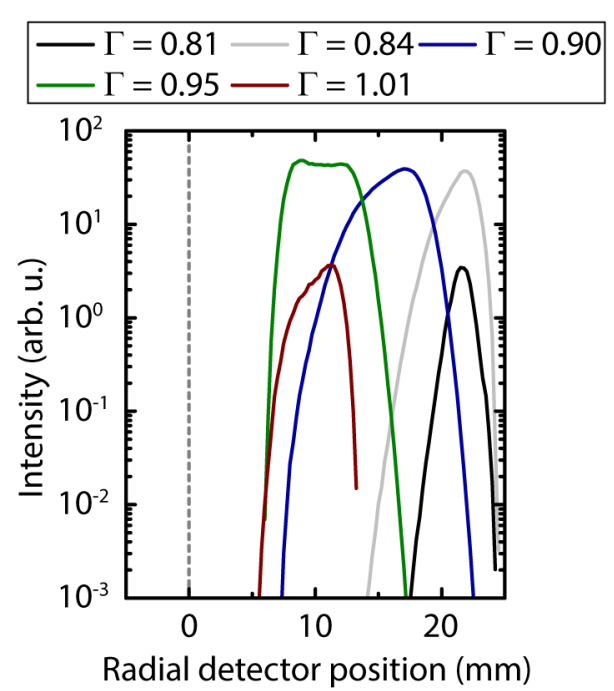

FiguRE 5.8: Cross section of the detector images with the assumption of radially symmetric electron emission for two different laser positions at the tip shaft (A: apex + $30 \mu \mathrm{m}, \mathrm{B}:$ apex $+60 \mu \mathrm{m})$.

detector, is nicely visible by comparing Fig. 5.8A and B. Fig. 5.8A shows the radial intensity distribution simulated for a laser position $30 \mu \mathrm{m}$ away from the tip apex, whereas Fig. 5.8B depicts the distribution for excitation of the shaft with $60 \mu \mathrm{m}$ distance to the apex. Between the curves for values of $\Gamma=0.90$ and $\Gamma=1.06$ in Fig. $5.8 \mathrm{~A}$ ( $\Gamma=0.81$ and $\Gamma=0.95$ in Fig. $5.8 \mathrm{~B}$ ), the maximum intensity is shifting towards the center. Whereas between $\Gamma=1.06$ and $\Gamma=1.12(\Gamma=0.95$ and $\Gamma=1.01)$, the intensity drops without a further shift of the signal towards the center, leaving an electron-free inner circle.

\subsubsection{Transition-region}

The transition region between apex and shaft emission is pictured in Fig. 5.9. The first row shows images acquired by illuminating the emitter apex. The next two rows show pictures for a laser position $10 \mu \mathrm{m}$ and $20 \mu \mathrm{m}$ away from the apex $(y=0 \mu \mathrm{m})$, respectively. Note that the distance the emitter is protruding from the suppressor aperture is usually around $250 \mu \mathrm{m}$ (270 $\mathrm{mm}$ for this specific emitter). The sketches illustrating the laser position and the potential lines in Fig. 5.9 are exaggerated.

Again, the difference in the emission pattern near the cutoff point for apex (Fig. 5.9A-I) and shaft (Fig. 5.9C-VI) electrons is clearly visible. A focusing of the ring or arc shape upon increasing $\Gamma$ parameter occurs at all laser positions. 


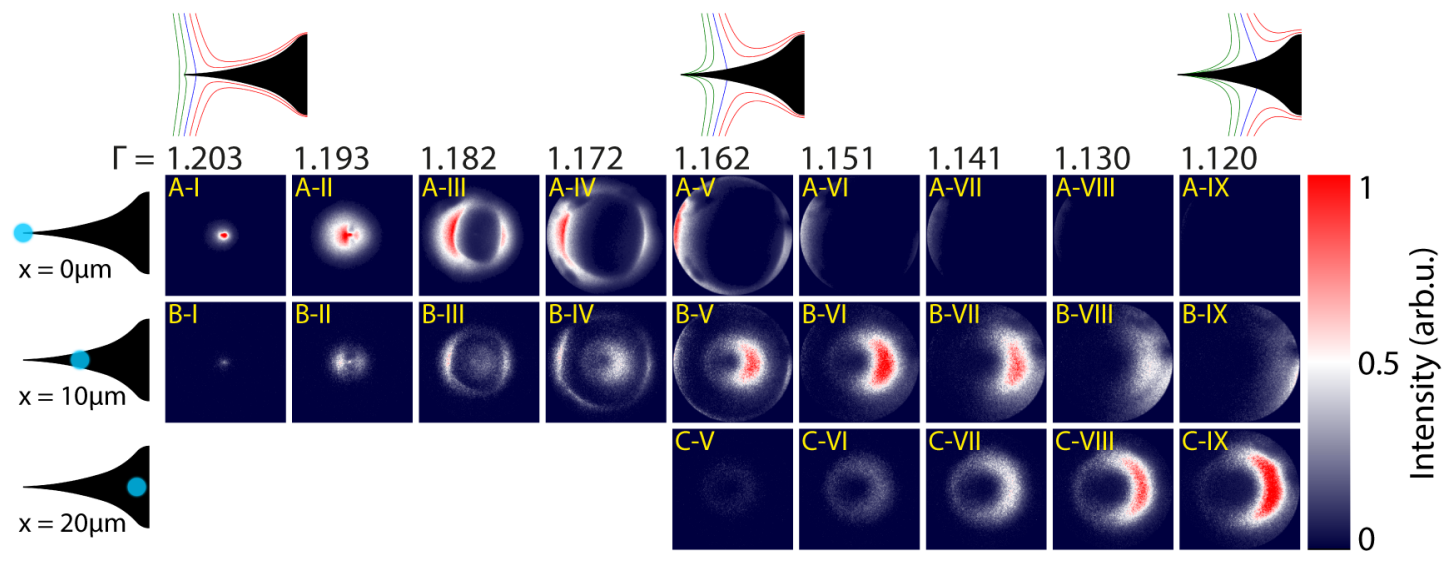

FiguRE 5.9: Detector images for three different laser positions and various voltage settings. The intensity is normalized across all pictures. Sketches (left and above) illustrate laser position and potential lines (not to scale).

An somewhat unexpected behavior is found when illuminating the tip at a distance $10 \mu \mathrm{m}$ from the apex position. In the range of $\Gamma=1.18$ until $\Gamma=1.16$, two features are visible simultaneously: The ring shape found when illuminating the apex and the arc shape from shaft illumination at $x=20 \mu \mathrm{m}$. The ring shape appears in the same size as for apex illumination but with lower intensity (e.g. comparison between Fig. 5.9B-IV and Fig. 5.9A-IV). The inner arc shape is slightly larger compared to the arc in Fig. 5.9C (e.g. Fig. 5.9B-VII vs. Fig. 5.9C-VII). This corresponds to an origin of the electrons closer to the apex as expected for this laser position. Additionally, the arc in Fig. 5.9B can be focused more tightly (Fig. 5.9B-III vs. Fig. 5.9C-VI) and is visible for higher $\Gamma$ values, which underlines that the electrons originate from a spot closer to the apex compared to the electrons from Fig. 5.9C.

The simultaneous presence of a ring and an arc feature, however, is difficult to interpret with the current knowledge about the tip shape and electron trajectories. One would expect that there is a signal in the intermediate range between the arc and the ring, as well. Contributions from a ghosting effect, which appears in later measurements, can also be ruled out. If that were the case, there should be a second signal in either Fig. 5.9C or Fig. 5.9A, and the diameter of the ring shape would decrease from Fig. 5.9A to Fig. 5.9B. Furthermore, following the measurements, the employed tungsten tip was investigated in a SEM and was found to be smooth in the range of $0-40 \mu \mathrm{m}$. A reasonable explanation includes, that the outer signal stems from electrons emitted at the apex, or in the vicinity of the apex. Since the laser focus size in this experiment is on the order of $20 \mu \mathrm{m}$, the laser intensity is still significant at the apex region, leading to 
a field-enhanced emission. Thus, the signal is expected to be lower than in the case of direct apex illumination. The inner feature in the detector pattern stems from regular shaft emission, and the area between the two features contains no signal due to the localization of the field enhancement to the apex region. This double-feature signal will later on be helpful for the investigation of the polarization dependence. It is, however, necessary to know from which region on the tip surface the electrons originate, which are forming the ring shape visible inFig. 5.9.

\subsubsection{Formation of the ring pattern}

The arc pattern observed on the detector under illumination of the tip shaft is easily explained with the trajectories shown in Fig. 5.2B. The trajectories which impinge on the detector closest to the symmetry axis stem from the tip surface near the cutoff point. Trajectories originating closer to the tip apex arrive with a larger distance to the symmetry axis. At a certain point, the trajectories are clipped by the extractor aperture. The resulting pattern on the detector therefore has a peak and results in an arc pattern. The width of the arc shape depends on the voltage setting and the laser focal size.

A more involved situation is found when illuminating the tip apex. In order to observe a ring pattern (arc pattern for blunter tips), the voltage ratio $\Gamma$ is required to be high $(\geq 1)$. A simulated electron distribution is shown in Fig. 5.10A. The graph shows the normalized electron count as a function of the radial detector position. In order to identify trajectories which cross the symmetry line (crossover trajectories), only the contribution from one side of the tip is shown. The green bar marks the part which corresponds to the ring feature. The remaining signal is marked with red color. In Fig. 5.10B and C, the link between the electron starting point on the tip surface ( $z$-component) and the radial impinging position at the detector is illustrated. Fig. 5.10B shows the relation for a fixed initial kinetic energy of $0.7 \mathrm{eV}$ for different emission angles $\alpha$, whereas Fig. 5.10C depicts the relation for different kinetic energies at a fixed emission angle. The dashed line shows the transition between apex and shaft regions. By comparing the position of the ring pattern in Fig. 5.10A with the curves in Fig. 5.10B, C, one can already deduce the origin of the electrons forming the ring feature. Note that the electron distribution is gained by weighting of the trajectories (see Sec.3.1.1). For this voltage setting, the 
A

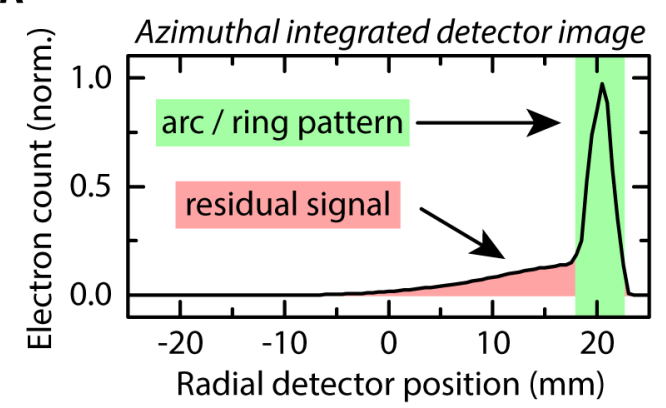

B

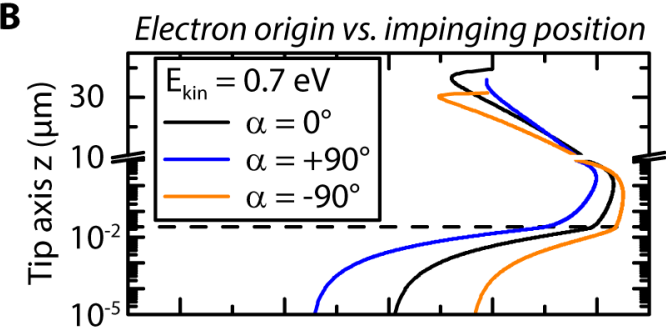

C

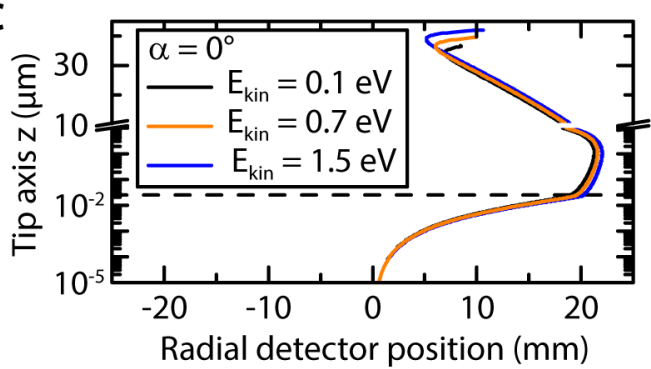

D

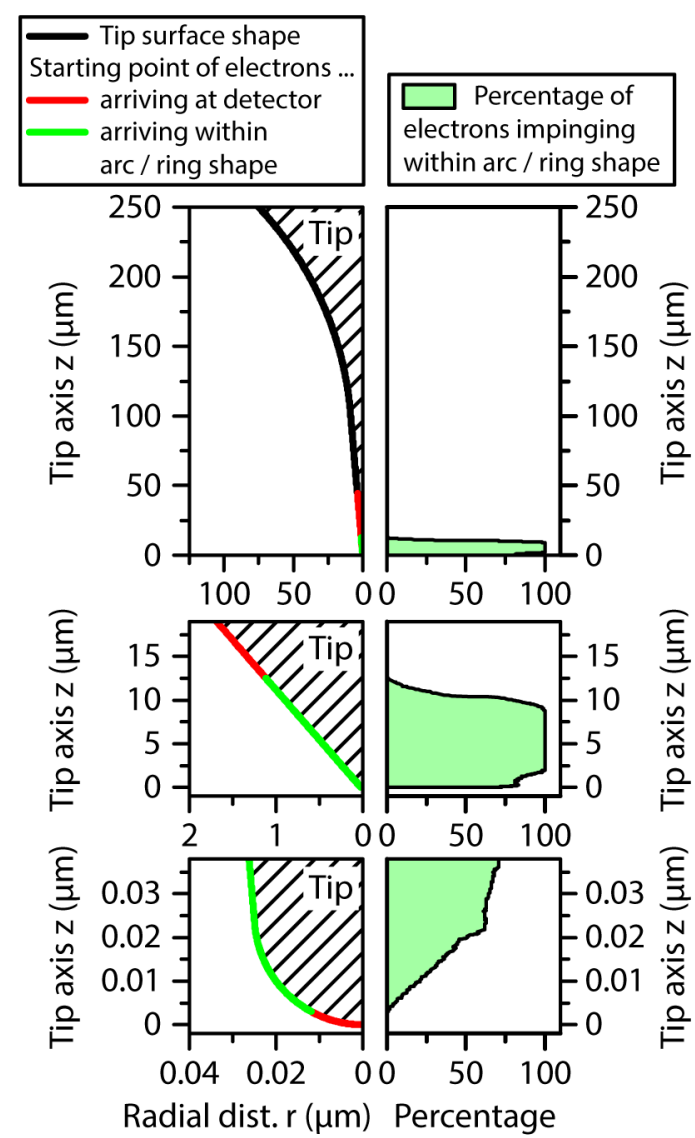

FIGURE 5.10: Relation between impinging position on the detector and origin on the tip surface of photoemitted electrons for illumination of the tip apex. A: Normalized radial electron distribution on the detector for $\Gamma=1.06$. Only electrons emitted from a half sphere are taken into account in order to identify crossover trajectories. B, $\mathrm{C}$ : $z$-component of electron origin on the tip surface versus radial impinging position on the detector for different starting angles at constant initial kinetic energy (B) and different kinetic energies for a constant angle (C). Dashed line displays transition from apex to shaft region. D: Tip shape at different magnifications. Red and green regions mark the areas, from which electrons reach the detector. Green regions depict the area, from which electrons impinge on the detector in the ring pattern. E: Percentage of the electrons contributing to the ring pattern as a function of starting positions.

electrons stem from a part of the shaft which extends up to $10 \mu \mathrm{m}$ from the apex. Furthermore, also a contribution from the apex is visible. For better visibility, the starting points of the electron trajectories which leads to the ring pattern are shown in Fig. 5.10D (green line) for different magnifications together with the tip surface (black line) and the residual trajectories which impinge on the detector at a different positions (red line). Furthermore, in Fig. 5.10E, the percentage of the electrons which contribute to the ring pattern is shown for the corresponding starting position on the tip surface. One can easily see that a part of the apex, as well as the nearby shaft, contributes to the ring pattern. 
In summary, the ring shape upon illumination of the apex is formed by electrons stemming from the tip shaft as well as the tip apex.

\subsection{Maps of electron emission sites}

In order to achieve knowledge about the emission sites of photoemitted electrons, raster scans are performed for various voltage ratios $\Gamma$.

\subsubsection{Two-dimensional raster scans}

By raster scanning the laser focus over the emitter, maps of the effective electron emission sites are recorded. Fig. 5.11 shows raster scans of two different tips for various voltage ratios $\Gamma$. In this experiment, the overall intensity on the detector is counted and the resulting maps are normalized in Fig. 5.11A and B, respectively. The images in Fig. 5.11A are obtained using a blunt tungsten tip with a diameter of about $400 \mathrm{~nm}$. In contrast, Fig. 5.11B shows scans for a sharp tip with $40 \mathrm{~nm}$ apex diameter. Blue regions in Fig. 5.11A correspond to laser positions which do not result in a signal on the detector. For a low value of $\Gamma$, mainly electrons extracted by illuminating the tip shaft are able to reach the detector. This area shifts towards the apex region for increasing $\Gamma$, which corresponds to a shift of the cutoff-point, as discussed in Sec. 3.2. The combination of maps for different voltage settings results in an image of the tip itself, convoluted with the laser focus size. The lower limit for the voltage ratio $\Gamma$ is mainly given by the onset of static field emission. The increase of the maximum signal towards high $\Gamma$-values is a result of the focusing effect and the associated clipping of the electron pattern at the detector (cf. Fig. 5.7A).

In Fig. 5.11B, a second contribution to the emission is visible. This signal is independent of $\Gamma$ and remains at the same position, and corresponds to electron emission from the tip apex. Hence, it is only visible for sharp tips, as substantial field enhancement required to obtain a signal of comparable intensity to the shaft contribution. The intensity of the apex signal is almost independent of $\Gamma$. This is expected, as an increase in $\Gamma$ only leads to a slight focusing of the electron trajectories (cf. 5.1). 

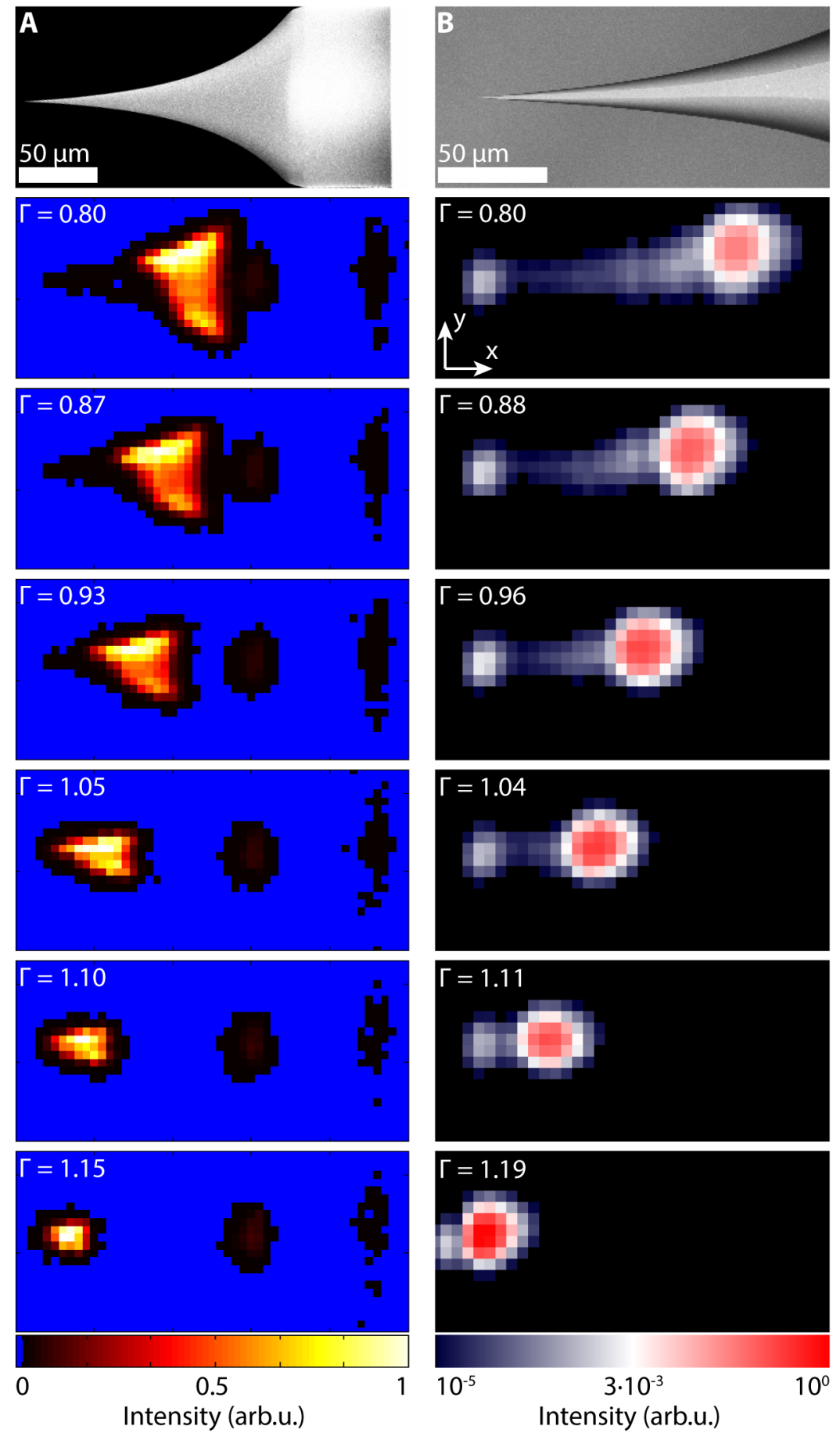

FiguRE 5.11: Intensity maps of the electron yield gathered by raster scanning the laser focus over the emitter for various values of $\Gamma$. The maps are measured using a A: blunt $\left(d_{\text {apex }} \approx 400 \mathrm{~nm}\right)$ and B: sharp tip $\left(d_{\text {apex }} \approx 40 \mathrm{~nm}\right)$. The first sub-figures show SEM images of the corresponding tips. 
A

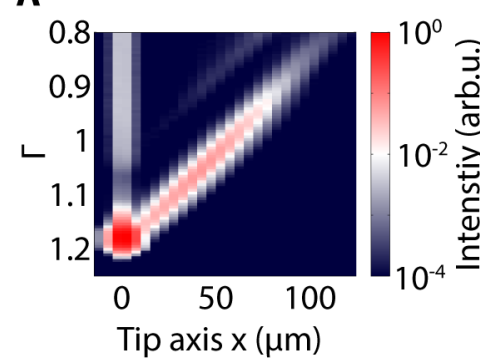

B

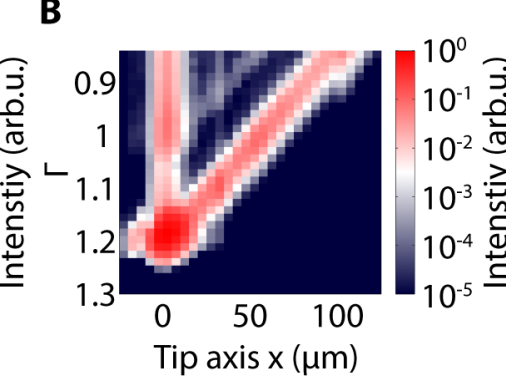

C

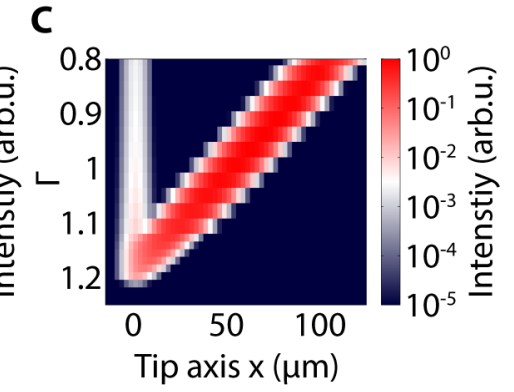

FiguRe 5.12: Electron signal as a function of voltage ratio $\Gamma$ and laser spot position along the tip axis z. A, B: experimental data of two different tungsten tips. C: Simulation results.

The combination of voltage setting and laser focus position enables the selection of an emission region from the macroscopic tip. It can be used to effectively separate apex from shaft emission. The separation of the two is important in order to achieve optimal performance in a UTEM, since the source size should be as low as possible while maintaining high electron currents and a short pulse duration. Electrons emitted from the tip shaft are undesired, as they drastically corrupt the source size, and hence increase the emittance.

\subsubsection{Laser focus position vs. voltage ratio $\Gamma$}

A distinction between shaft electron yield and the apex signal is again visible in Fig. 5.12 (A and $\mathrm{B}$ are measurements, $\mathrm{C}$ depicts simulated data). Here, the $z$-axis corresponds to the laser position along the tip axis. In the orthogonal direction ( $y$-axis in Fig. 5.11), the laser focus is centered with respect to the tip emitter using the emitter map gained in Fig. 5.11. Again, the apex contribution is clearly visible at the tip axis position $(z=0 \mu \mathrm{m})$ and is not shifting upon changes in $\Gamma$. The shaft contribution, on the other hand, is evident as a diagonal line. The signal from shaft electrons is decreasing in intensity for decreasing values of $\Gamma$. This effect is, as explained before, a result from the spatially increasing pattern of the electron signal on the detector and the subsequent clipping due to the limited detector size (cf. Fig. 5.7A).

At the apex position, if $\Gamma$ is decreased, as discussed in 5.2, the ring pattern (arc pattern for blunt tips) disappears from the detector, and a homogeneous electron distribution is observed. However, an intermediate region is found in the measured data, in which the ring is not observed anymore but the uniform pattern is not yet intense. This 
intermediate region can be seen in Fig. 5.12A for values between $\Gamma=1.1$ and $\Gamma=1.05$ at $z=0 \mu \mathrm{m}$, and can be explained considering the field enhancement at the tip apex. The static field-enhancement is suppressed at the field reversal point and subsequently increases for decreasing values of $\Gamma$. As a consequence, between the $\Gamma$ value where the ring shape disappears from the detector and the onset of the homogeneous pattern, the field enhancement is not yet large enough to allow for the same intensity in electron emission as for lower voltage ratios.

A large agreement is given when comparing simulated (Fig. 5.12C) with measured (Fig. 5.12A, B) data. In particular, the apex and shaft signals follow the same general behavior. Only the enhanced emission near the field reversal point is weaker in the simulations.

\subsubsection{Double image}

The second diagonal line in Fig. 5.12A stems from a ghosting effect of the incident light at the sapphire window of the vacuum chamber (Fig. 5.13). Assuming that the laser light impinges on the glass with an angle $\alpha$, the distance $L$ between the original transmitted light and a doubly reflected beam can be calculated using Snell's law to

$$
L=\cos (\alpha) \cdot 2 \cdot \tan \left(\arcsin \left(\frac{\sin (\alpha)}{n_{2}} n_{1}\right)\right) \cdot d
$$

The window thickness is $d=3 \mathrm{~mm}$, and the refractive index of sapphire for a laser wavelength of $400 \mathrm{~nm}$ is $n_{2}=1.7865$ [154]. Assuming an incident angle of $\alpha=1^{\circ}$ results in a lateral distance of about $58 \mu \mathrm{m}$ ( $n_{1}$ set to unity). This is approximately the distance found in Fig. 5.12A between the two diagonal signals (the distance in $\mathrm{x}$-direction between the two features). For a given tip axis position, the second signal is visible for a lower $\Gamma$. Hence, the ghosting signal is shifted towards the tip shaft.

\subsubsection{Benefit for the UTEM}

Considering the alignment of an ultrafast TEM, large raster scans or scans of the voltage ratio are not feasible. However, locating the position of the apex with a single electron image is an involving task. Utilizing the features shown above, one can easily locate the laser focus position on the tip. In case the laser focus is at the tip apex position, the electron beam should not move upon change of the suppressor voltage (thus changing 


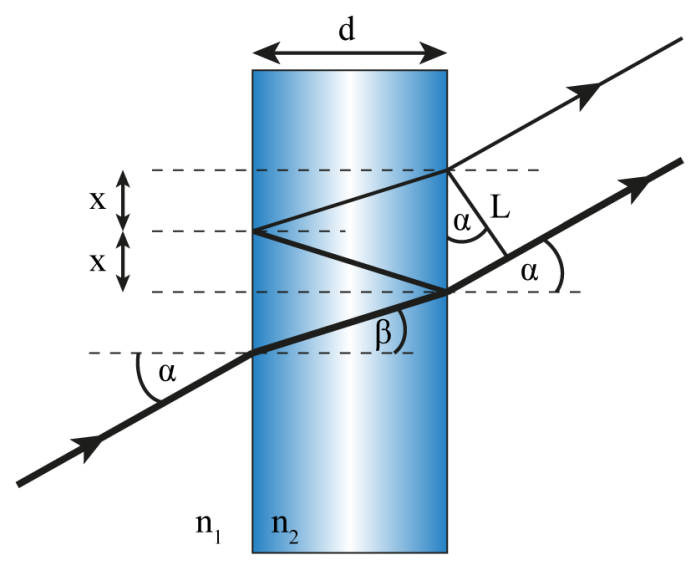

FiguRE 5.13: Sketch of beam paths through a glass window illustrating the ghosting effect.

$\Gamma)$, but instead (de-)focus. Furthermore, the intensity of the signal should remain almost constant during this process.

Near the field reversal setting $(\Gamma \approx 1.2)$, the detected electrons originate from the apex itself and from the apex-near region of the tip shaft (cf. Sec. 5.2.4). In this setting, the source size is increased due to the shaft contribution and therefore corrupts the spatial coherence (cf. Sec. 2.4.3) and the electron pulse duration. Latter stems mainly from different path lengths between electrons starting at the tip apex and such starting at the tip shaft, resulting in a time-of-flight difference. Although this "high- $\Gamma$ "-mode is not the favorable setting for high magnifications, it might still be useful for lower magnifications. It is clearly visible in Fig. 5.11B or Fig. 5.12A, B, that the overall electron yield for a setting close to the field reversal is two to three orders of magnitude larger compared to electron emission from the apex for lower $\Gamma$. Hence, a setting with $\Gamma \approx 1.2$ can be used as a high current low, magnification mode which can be useful for, e.g., finding the relevant structure for an investigation.

\subsection{Polarization dependence}

The electron yield via nonlinear photoemission should strongly depend on the polarization when illuminating a sharp metal tip. However, an effect in the emission pattern is barely noticeable. By comparing the upper and lower row in Fig. 5.14, this becomes evident. The upper row (Fig. 5.14A) shows detector images for different voltage ratios with a polarization vector of the laser light along the tip axis. Images with a polarization 


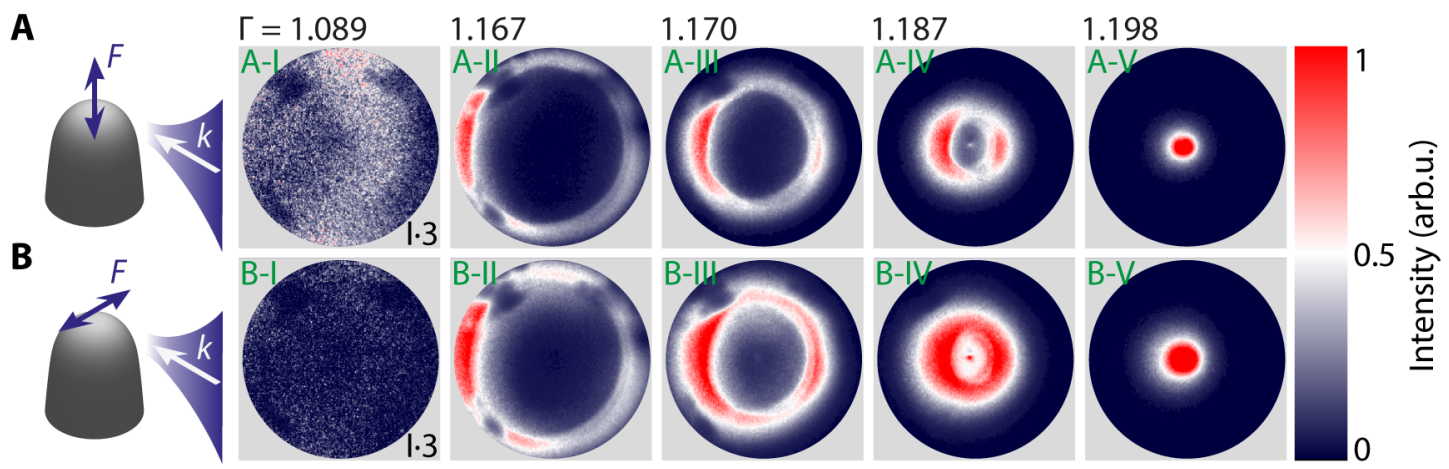

Figure 5.14: Electron signal for illumination of the tip apex with polarization vector along (A) and perpendicular (B) to the tip axis for different voltage ratios $\Gamma$. The intensity is normalized across all pictures, i.e. not to the maximum within each individual image. For $\Gamma=1.089$, the intensity is multiplied by a factor of three.

vector perpendicular to the tip axis are depicted in Fig. 5.14B. It should be noted that the laser pulse energy in the lower row was $9 \%$ larger compared to the measurements with parallel polarization. The images in Fig. 5.14 are measured by illuminating the tip apex. A significant difference in the spatial distribution of electrons is not visible. However, for a low voltage ratio $\Gamma$ (leftmost picture) the intensity is higher when the polarization vector is parallel to the tip axis. The following arguments can be used to explain this behavior.

Due to the geometric field enhancement (lightning rod effect, cf. Fig. 2.6) in combination with the optical field enhancement, a significant difference in the electron count rate between the two polarization directions is expected. However, even though largest right in front of the apex, the field enhancement is not negligible in the vicinity of the apex (cf. Ref. [106]). Also, the static field enhancement can be reduced by a suitable suppressor voltage which is the case for the patterns shown in Fig. 5.14(II-V). This would, however, explain why the intensity on the detector is the same. Considering the emission area, the intensity for polarization perpendicular to the tip axis could even be higher in that case.

Fig. 5.15 shows polarization dependent measurements of the electron currents for different voltage settings and positions of the laser focus on the tip. The curves in Fig. 5.15A and $\mathrm{B}$ are obtained under illumination of the tip apex, and in Fig. 5.15C the laser focus was on the shaft with a distance of $30 \mu \mathrm{m}$ from the apex. Fig. 5.15A and B are measured for two different values of $\Gamma$. The plot in Fig. 5.15A corresponds to a setting of a low $\Gamma$ value with a homogeneous emission pattern (cf. Fig. 5.14A), and the curve in Fig. 5.15B 

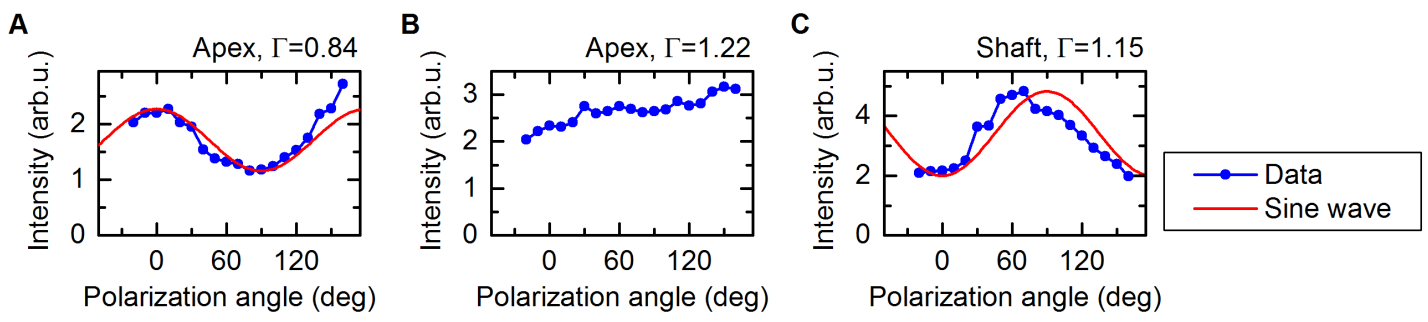

Figure 5.15: Polarization-dependent measurement of the electron yield for different laser positions and voltage settings. A polarization angle of zero degrees corresponds to a polarization vector pointing along the tip axis. Sine waves are added in red for comparison. A, B: Illumination of the apex and a voltage ratio of $\Gamma=0.84$ (A) and $\Gamma=1.22(\mathrm{~B})$. C: Illumination of the shaft $($ apex $+30 \mu \mathrm{m})$ and a $\Gamma$-value close to the cutoff-point.

to a $\Gamma$ value near the cutoff point where the ring shaped pattern is observable. A polarization angle of zero degrees denotes that the polarization vector points along the tip axis. As expected, the electron emission from the tip apex follows a sinusoidal curve as a function of polarization direction with a maximum signal for polarization along the tip axis. In contrast, electron emission from the shaft follows the same behavior but with a maximum shifted by $90^{\circ}$ in the polarization vector angle, which corresponds to a rotation of $45^{\circ}$ of the lambda half wave-plate. In Fig. 5.15C, the maximum signal is at about $70^{\circ}$ to $80^{\circ}$ instead of $90^{\circ}$. This is, however, not surprising considering the shape of the tip shaft. In a distance of $30 \mu \mathrm{m}$ from the tip apex, where this curve was measured, the tip surface is not parallel to the tip axis but has an angle of about $5^{\circ}$ to $10^{\circ}$ with respect to the tip axis (see Fig. 4.2B). This results in a shift of the maximum signal in the measurements, since the maximum signal is obtained for a polarization direction perpendicular to the surface.

A significantly different signal is received in Fig. 5.15B. The curve stems from the measured ring shape which is visible for a large voltage ratio $\Gamma$ upon apex illumination. As revealed in Fig. 5.2A, for this setting, a distinction between apex and shaft electrons is not possible when averaging the electron signal over the detector. The combination of apex and shaft electron dependence on the polarization cancel each other out, which is why no polarization dependence is observable. 



\section{Chapter 6}

\section{Gun performance capabilities}

In this chapter, characteristics of the electron gun are shown continuing the measured and simulated results presented in the previous chapter. Parameters such as the transmission of generated electrons through the electron gun, the emittance and pulse duration are extracted from the simulated data. In the previous chapter, very good agreement between simulation and experiment was found with regard to the spatial electron distribution. Based on this conformity, further simulations are carried out confidently assuming similar agreement with the experiment.

\subsection{Transmission}

At first, the transmission of electrons through the first apertures is considered. In static electron emission mode, most of the electrons are already blocked by those apertures, which is evident by comparing the emission current (current through the emitter tip) with the beam current (current at detection plane). The former is on the order of $100 \mu \mathrm{A}$, whereas the latter is in the range of $300 \mathrm{pA}-10 \mathrm{nA}^{1}$. However, in the case of ultrafast microscopy with only $1-100$ emitted electrons per laser pulse, such a high loss rate cannot be afforded. Hence, the question arises, how many electrons emitted from the tip are able to pass through the electron gun.

To investigate the transmission, the geometry consisting of tip, suppressor and extractor is used, and a virtual aperture is introduced at the detection plane (cf. Fig. 4.4A). The

\footnotetext{
${ }^{1}$ Data taken from JEOL manual and acceptance report of the employed JEOL-2100F microscope, respectively.
} 
A

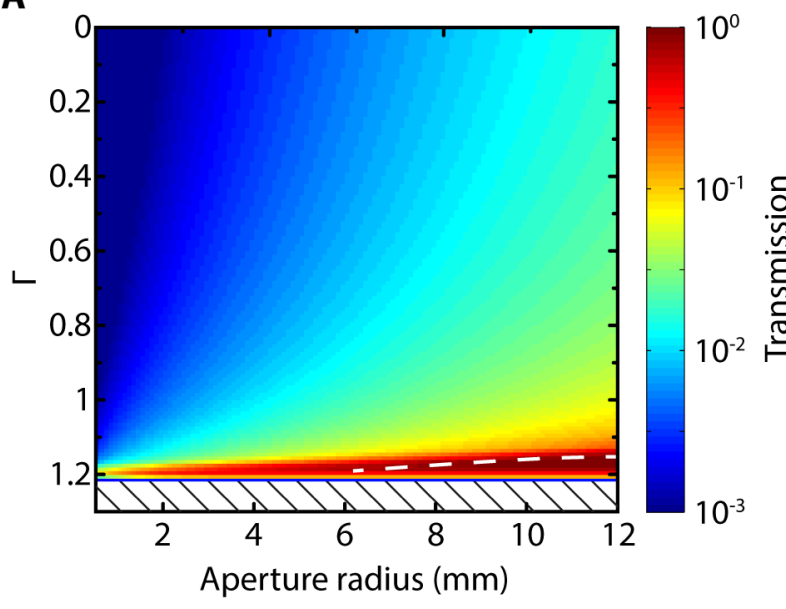

B
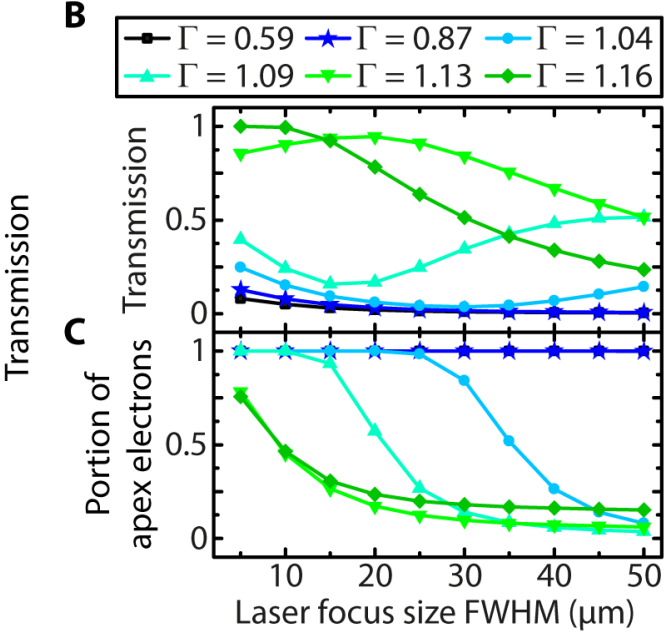

FIGURE 6.1: Transmission of electrons through the extractor electrode and a virtual aperture at the detection plane. The transmission corresponds to the fraction of incident electrons to the number of emitted electrons. A: Transmission as a function of aperture radius and $\Gamma$. Below white dashed line: $100 \%$ transmission. Shaded area at the bottom: Suppressed electron emission due to field reversal. B: Transmission as a function of laser focus size for different voltage ratios. C: Relative contribution of apex electrons to the overall electron count at the detector.

suppressor voltage is fixed at $U_{\text {sup }}=-1000 \mathrm{~V}$, and the extractor voltage at $U_{\text {ext }}=0 \mathrm{~V}$. Varying the tip bias voltage leads to different values of $\Gamma$. The emission angle with respect to the surface normal is again equally distributed in the range of $-90^{\circ}$ to $90^{\circ}$. The initial kinetic energy is modeled with a Gaussian distribution centered at $E_{0}=0.75 \mathrm{eV}$ with $\sigma_{E}=0.32 \mathrm{eV}$. For this calculation, the laser focus position is set to the apex position, since this is the desired mode of operation. The transmission is given by the ratio of electrons impinging on the detection plane to the number of electrons emitted from the tip. The result is visible in Fig. 6.1A. The aperture radius belongs to a virtual aperture placed directly in front of the detector.

Originally, an additional electrode belongs to the electron gun as well, the focus lens with phosphor screen. Due to complexity, this electrode is not implemented in the measurements and simulations of this setup. In order to obtain a better comparison between the simulation and measurements conducted within the UTEM, this focus electrode is considered as an aperture. The size of the virtual aperture, which corresponds to the projected aperture of the focus lens, has a radius of $2 \mathrm{~mm}$ at the detection plane of the simulation. Therefore, this value is used as an aperture radius if not stated otherwise.

The effect of varying the aperture radius is visible in Fig. 6.1A. By decreasing the aperture radius, as expected, the transmission decreases by cutting off more electrons at 
the aperture. For a lower voltage ratio $\Gamma$, the transmission is reduced as well. This is explained with the observed focusing effect for increasing $\Gamma$ found in the previous chapter, i.e., the transmission is lower due to a defocused electron beam upon decreasing $\Gamma$. Considering an aperture radius of $2 \mathrm{~mm}$ for example, the transmission increases from $3 \cdot 10^{-3}$ for $\Gamma=0$ to 0.4 for $\Gamma=1.19$.

Below the white dashed line in Fig. 6.1A, the transmission is unity (100\%), as for these voltages, all trajectories from shaft and apex are bent strongly enough to pass the extractor aperture and subsequently the aperture at the detector position.

Fig. 6.1B depicts the transmission for different values of $\Gamma$ as a function of the laser focus size. For low $\Gamma$, a decrease in the transmission is observed for increasing focus size. However, in the case of $\Gamma$-values close to the cutoff point, an increase is visible as soon as shaft electrons become present at the detection plane. This reflects the shift in the weighting between shaft and apex electrons for increasing focus size. In the results shown here, the center of the laser focus is fixed at the apex position. Therefore, an increase of the focus size leads to a higher weighting of shaft electrons compared to apex electrons. Thus, the transmission increases once electrons form the tip shaft pass through the apertures. This can be verified by comparing the onset of increasing transmission with the onset of the decrease of the ratio between apex and shaft electron count shown in Fig. 6.1C.

\subsection{Emittance}

For the description of an electron microscope, the emittance is an important figure-ofmerit. The emittance allows for a determination of the achievable focus size for a given illumination angle and imaging setup.

Fig. 6.2 depicts the normalized RMS-emittance (cf. Sec. 2.4.1) calculated from the numerical simulations. In accordance with the transmission (Fig.6.1A), the emittance decreases for smaller apertures and smaller $\Gamma$ (Fig.6.2A). A major increase is visible for high values of $\Gamma$, where electrons emitted from the tip shaft impinge on the detector as well, thus drastically deteriorating the emittance. Values of the emittance vary from $1 \mathrm{nmmrad}$ (e.g. $\Gamma=0.56,0.5 \mathrm{~mm}$ aperture radius) to almost $10^{5} \mathrm{~nm} \operatorname{mrad}$ (e.g. 
A

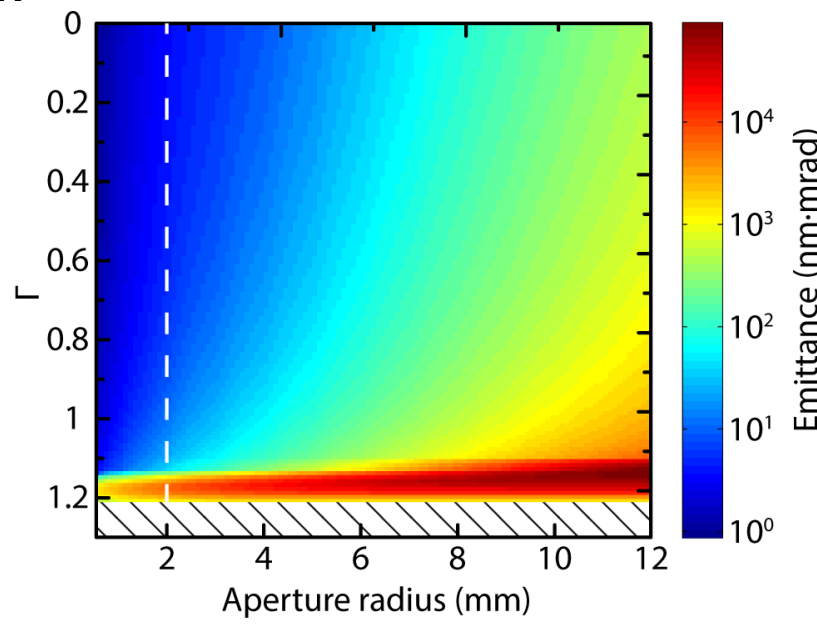

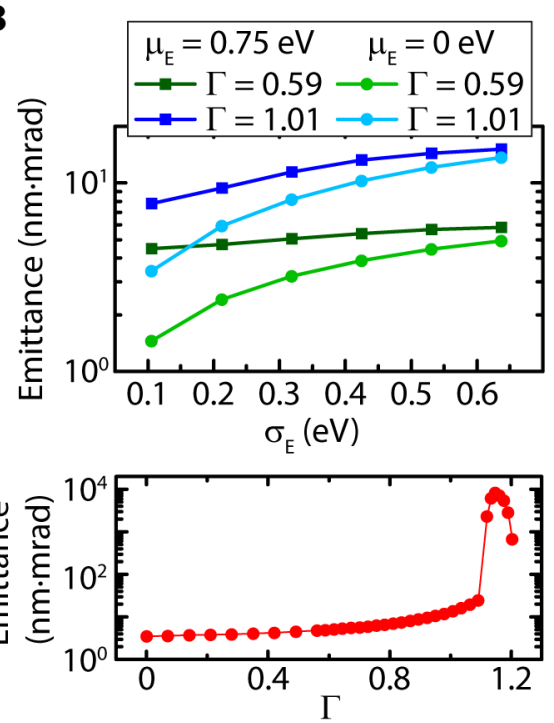

FiguRE 6.2: Normalized RMS-emittance. A: As a function of aperture radius (aperture positioned directly in front of the detector, cf. Fig. 5.2) and voltage ratio $\Gamma$. Gaussian distribution of initial kinetic energy distribution with $\sigma_{E}=0.32 \mathrm{eV}$ and $\mu_{E}=0 \mathrm{eV}$. Shaded area: No electron emission possible. B: Emittance depending on the standard deviation of the initial kinetic energy distribution for two voltage ratios (color coded) and two means of the electron distribution (symbol coded). Aperture radius is set to $2 \mathrm{~mm}$. C: $\Gamma$-dependence of the emittance for an aperture radius of $2 \mathrm{~mm}$ (corresponding to the white dashed line in A).

$\Gamma=1.12,12 \mathrm{~mm}$ aperture radius). In Fig. $6.2 \mathrm{C}$, a cross section along an aperture diameter of $2 \mathrm{~mm}$ is plotted. The increase of the emittance for increasing $\Gamma$ is nicely visible, again. For $\Gamma \geq 1.1$, electrons originating from the tip apex as well as the apex-near shaft region are bent onto the detector and through the aperture. Hence, the emittance experiences a steep increase.

In Fig. 6.2B, the dependence on the standard deviation of the initial kinetic energy $\left(\sigma_{E}\right)$ is plotted. Blue and green colors correspond to a $\Gamma$ of 1.01 and 0.59 , respectively. Squares and circles indicate the different means $\left(\mu_{E}\right)$ of the assumed Gaussian distribution. It is evident that for a broader distribution, the emittance becomes larger. This broadening effect is more pronounced for a mean of $0 \mathrm{eV}$. The ratio of the emittance between $\sigma_{E}=0.64$ and $\sigma_{E}=0.1$ is a factor of approximately 2 for $\mu_{E}=0.75 \mathrm{eV}$, and a factor of 4 for $\mu_{E}=0 \mathrm{eV}$. A reduction of the electron excess energy at the photoemission process offers the possibility to reduce the emittance, even though the effect is less than one order of magnitude.

The energy distribution function of photoemitted electrons usually depends on the excess energy and the density of (filled) electron states overlapped by a background signal 
of inelastic scattered electrons. In a two-photon photoemission process with a photon energy of $3.1 \mathrm{eV}$ and a work function of $4.5 \mathrm{eV}$ (tungsten), the maximum excess energy is $1.7 \mathrm{eV}$. In case of tungsten, the density of states down to $1.7 \mathrm{eV}$ below the Fermi energy is almost constant, and thus the energy distribution after photoemission is expected to be the same [155]. However, for linear and nonlinear photoemission with femtosecond laser pulses, different spectra are given throughout literature. Wytrykus et al. illuminated a thin gold film with $800 \mathrm{~nm}, 400 \mathrm{~nm}$ and $266 \mathrm{~nm}$ laser light corresponding to a three, two and one photon photoemission process, respectively [156]. The electron spectrum was found to fit an exponential function for nonlinear emission and a Gaussian distribution for single-photon emission. In all cases the maximum of the functions was set to $0 \mathrm{eV}$. In case of photoemission with a large bias voltage, Yanagisawa et al. found that the electron energy distribution has peaks at intervals of the photon energy, which can be approximated by Gaussian distributions [139]. However, in this case, the emission process is photon-assisted tunnel-emission rather than pure nonlinear photoemission. Since, in the experiments within this thesis, the energy distribution is expected to be constant up to the maximal excess energy (see above) and is usually assumed and measured as a Gaussian distribution in the literature [34, 35, 92], it is modeled as a Gaussian distribution with a mean of $0 \mathrm{eV}$ and standard deviation of $0.32 \mathrm{eV}$.

\subsection{Temporal properties}

The pulse duration of the generated electron pulses is one of the most important properties of an ultrafast TEM, as it ultimately limits the achievable time resolution. By using a femtosecond laser system with a pulse duration of $30 \mathrm{fs}$ for the generation of electrons, this value gives a lower bound of the electron pulse duration. However, different starting positions of the electrons on the tip surface and hence different distances yield a time difference which leads to a further increase of the pulse duration. In the simulations shown here, the electron emission time is assumed to be equal for all electrons, an assumption which corresponds to an instantaneous emission. The resulting values of the pulse duration thus have to be convoluted with the respective laser pulse duration of the experimental setup in order to give more realistic values. However, since the simulated pulse duration is significantly larger $(\sim 1 \mathrm{ps})$ than the laser pulse duration $(\sim 30 \mathrm{fs})$, the effect can be neglected for now. The pulse durations presented here are calculated 
for the prototype setup. In the UTEM setup, already after the extractor electrode, the electrons will be strongly accelerated (by the focus electrode with $U_{\text {foc }} \approx 7-9 \mathrm{kV}$ ). This additional fast acceleration after the extractor electrode will lead to a smaller temporal dispersion and thus shorter electron pulses. However, the behavior with regard to changing voltage settings of the electron gun is expected to be similar.

The electron pulse duration $\sigma_{t}$ is calculated by the second moment of the electron time of flight distribution:

$$
\sigma_{t}^{2}=\iiint d E_{0} d r d z\left(t\left(E_{0}, r, z\right)-t_{\text {mean }}\right)^{2} G\left(E_{0}, r, z\right),
$$

where $t\left(E_{0}, r, z\right)$ is the time of flight depending on the initial conditions, $t_{\text {mean }}$ the respective mean value and $G\left(E_{0}, r, z\right)$ the probability density function as introduced in Sec. 3.1.1.

The pulse duration calculated from the simulated trajectories are shown in Fig. 6.3, computed as a function of aperture radius and voltage ratio $\Gamma$. The plots to the right and at the bottom show cross sections in the respective directions. Again, the voltages at the suppressor and extractor are fixed $(-1000 \mathrm{~V}$ and $0 \mathrm{~V}$, respectively) and the variation of $\Gamma$ stems from varying the tip voltage. Furthermore, the center of the laser focus is set to the tip apex and a standard deviation of $\sigma_{z}=10 \mu \mathrm{m}$ is applied.

For an aperture radius of $2 \mathrm{~mm}$, the pulse duration rises from $640 \mathrm{fs}$ at $\Gamma=0$ to $1.2 \mathrm{ps}$ at $\Gamma=1.12$ before significantly increasing to $9 \mathrm{ps}$ at $\Gamma=1.17$. For even higher values of $\Gamma$, the pulse duration drops again, which is due to the suppression of electron emission when the cutoff-points shifts towards the tip apex. In case of a fixed voltage ratio $\Gamma$, the pulse duration rises with increasing aperture radius, as more electrons from different regions of the tip are collected. Eventually, a saturation sets in (Red line at the bottom plot of Fig. 6.3) when the voltage ratio is high and the aperture is larger than the maximum angle electrons are emitted into (cf., e.g., Fig. 5.2C). Most interestingly, the pulse duration is not significantly rising for increasing $\Gamma$, as long as shaft electrons are not bent through the apertures. This is important because the transmission and thus the overall electron count increases for larger $\Gamma$ (see Fig. 6.1A). Therefore, the UTEM can be run with a large current at high values of $\Gamma$ without losing temporal resolution. 


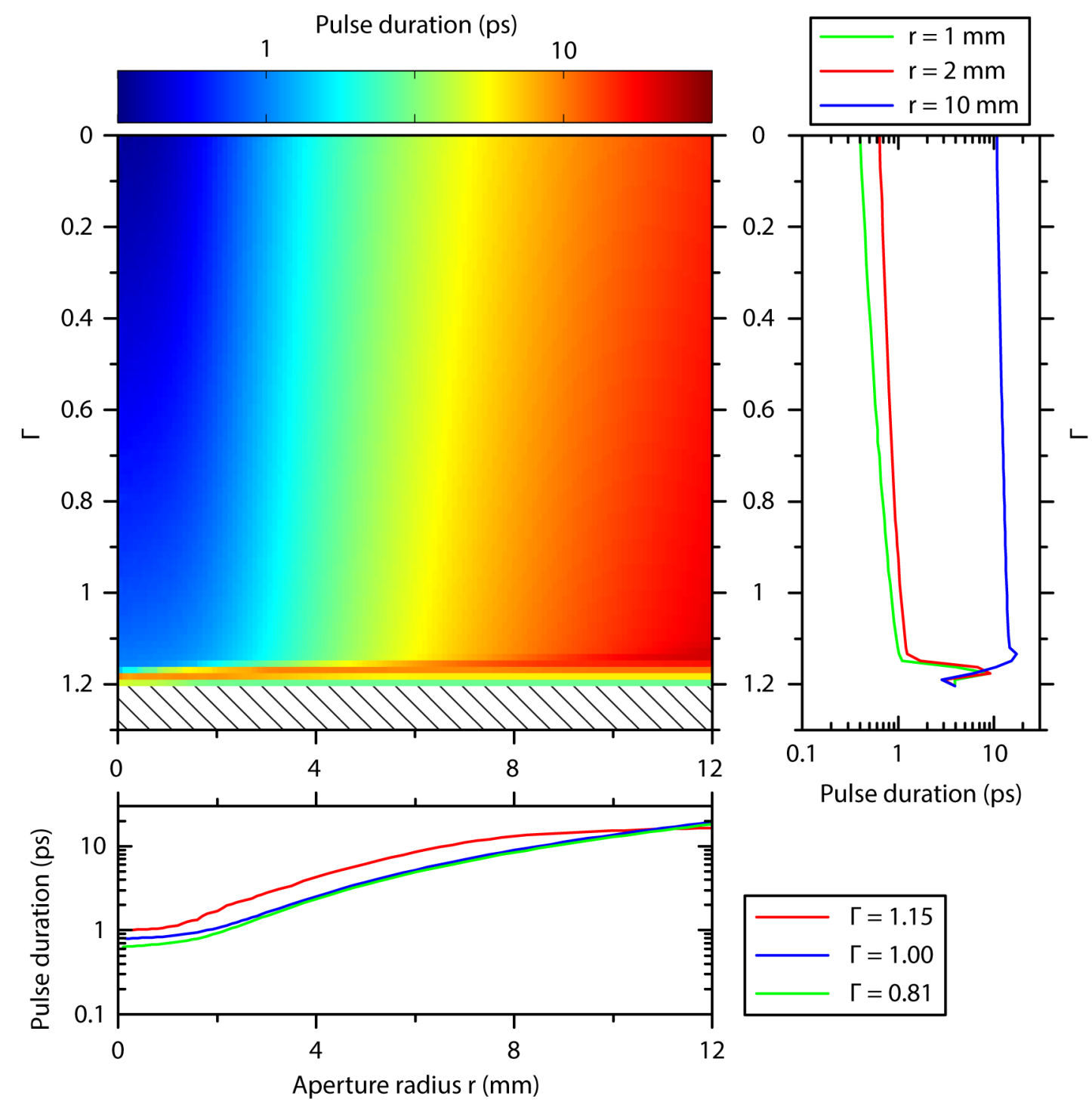

FiguRE 6.3: Calculated pulse duration from numerical simulations depending on $\Gamma$ and for apertures positioned at the detection plane, $75 \mathrm{~mm}$ away from the tip apex.

The plots at the bottom and to the right show cross-sections.

\subsubsection{Impact of the initial kinetic energy spread}

A further reduction of the pulse duration can be achieved by minimizing the velocity spread of the electrons, which stems from the initial kinetic energy distribution. Fig. 6.4 shows the dependence of the achievable pulse duration as a function of the standard deviation of the energy distribution for an aperture radius of $2 \mathrm{~mm}$ and a voltage ratio of $\Gamma=1$. However, the gain in pulse duration between a standard deviation of $0.4 \mathrm{eV}$ and $0.05 \mathrm{eV}$ is only a factor of two. Nonetheless, reducing $\sigma_{E_{0}}$ also has a positive effect on the emittance. 


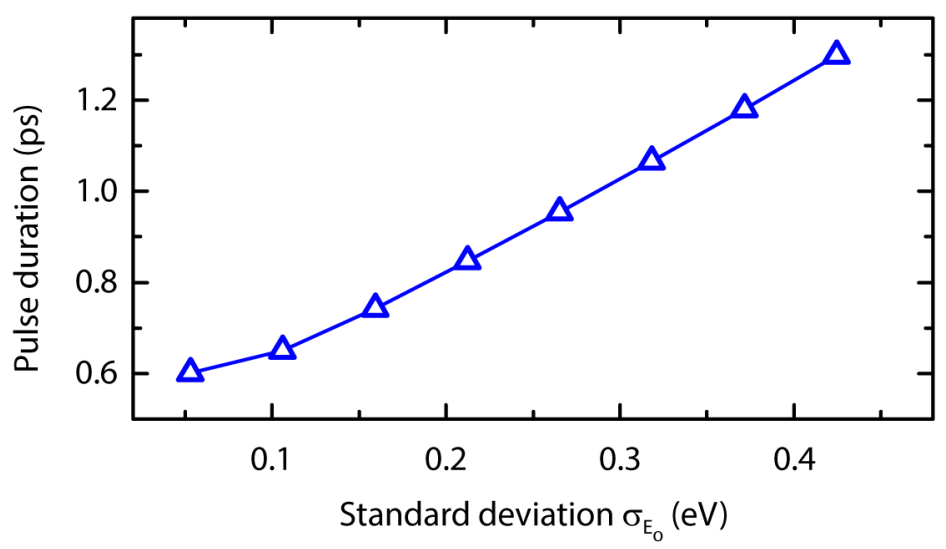

FIgURE 6.4: Pulse duration as a function of the standard deviation of the initial kinetic energy $\sigma_{E_{0}}$ with a mean of $0 \mathrm{eV}$. The aperture radius is $2 \mathrm{~mm}$ and the voltage ratio $\Gamma=1$.

\subsubsection{Considerations regarding the reduction of the kinetic energy spread}

The initial energy spread is, among others things, affected by the spectral width of the exciting laser, variations in the electron momentum within the metal, non-perpendicular emission to the metal surface (thus different velocities in the forward direction), and thermal blurring of the Fermi-Dirac distribution of the electron gas at room temperature.

The latter is even further increased due to the ultra-short laser pulse $(\sim 30 \mathrm{fs})$ excitation, which is shorter than the electron-phonon coupling time and therefore leads to a high electron gas temperature during the emission process. The temperature is expected to be about $1000 \mathrm{~K}[35,146]$. It can be further increased by cumulative heating of the photocathode due to a high repetition rate of the laser system. The high electron gas temperature then results in thermally assisted photoemission, which leads to a broadening of the initial electron energy distribution. In order to compensate at least for the cumulative heating, the emitter can be cooled, e.g. with liquid nitrogen [39]. However, the repetition rate in the experiments conducted within this work are relatively low $(250 \mathrm{kHz})$, thus cumulative heating can be excluded.

Adapting the wavelength of the laser illuminating the tip also leads to a control over the excess energy of emitted electrons. The wavelength can be controlled by implementing an optical parametric amplifier (OPA). An advantage of adjusting the laser wavelength is that the path difference between trajectories of electrons emitted non-perpendicularly to the surface is not as large compared to high excess energies, as electrons are easily 
accelerated away from the surface (see Fig. 5.10B and appendant text). However, the wavelength has to be adapted whenever the voltage settings at the electron gun are changed, as this changes the effective work function (cf. Eq. 2.3).

Reducing the initial kinetic energy spread can also be achieved by changing the effective work function of the material. The work function can be directly reduced by using a different material or by coating with another material (e.g. $\mathrm{ZrO}_{2}$ on tungsten). Concerning the coating with $\mathrm{ZrO}_{2}$, the emitter is heated to at least $1500 \mathrm{~K}$. In order to obtain a lower kinetic energy spread with regard to the thermally assisted photoemission, the emitter has to be cooled down after the $\mathrm{ZrO}_{2}$-layer buildup. First measurements indicate that the layer remains upon laser illumination. The effective work function can also be reduced by applying different static voltages. As pointed out in the previous chapters, the voltage ratio within the electron gun is essential. In order for the voltage ratio $\Gamma$ to remain constant, increasing voltages is only possible by a multiplicative factor, i.e. by changing the voltages of all electrodes. Precaution is necessary, as a high voltage difference between suppressor and tip can lead to a sparkover, even though the DC breakdown potential is on the order of $40 \mathrm{kV} / \mathrm{mm}$ for ultra-high vacuum (UHV) conditions [157]. With a gap between the tip and suppressor electrode of only $75 \mu \mathrm{m}$ (cf. Fig. 4.3), this corresponds to $3 \mathrm{kV}$. However, a discharge might appear already at lower voltages due to the relatively rough tip surface.

\subsubsection{Further Considerations regarding the reduction of the electron pulse duration}

Aside from directly adjusting parameters of the emission process, the pulse duration can also be modified after the generation of the electron pulse, e.g., by implementing a radio frequency compressor $[120,121,158]$. This is especially interesting beyond the single-electron limit in order to compensate for (temporal) space charge broadening of the electron pulse. Another approach is to use an so-called omega filter, which is originally placed between intermediate and projector lenses of an TEM to separate inelastically scattered electrons from elastically scattered and unscattered electrons in order to increase image contrast and resolution [46, 128, 159, 160]. To this end, the electrons are directed on an omega-shaped path by at least four sector magnets [161]. Using such a device above instead of beneath the specimen would have two benefits: 
First, the electron energy can be filtered, compensating for the emission process. Second, the electron pulse can be chirped due to the different path lengths of electrons with different kinetic energies. This may drastically shorten the pulse duration. Furthermore, the pulse duration could be compensated in a way that the pulse duration is lowest at the place of the specimen.

Georg Herink et al. recently demonstrated spectral compression of electrons pulses using an optical THz pulse [147, 162, 163]. Upon simultaneous illumination of a metal needle emitter with a near-infrared and a THz laser pulse, the electron emission is streaked and gated due to the modification of the metal potential barrier and the local electric field. For an appropriate time delay between $\mathrm{THz}$ and $\mathrm{NIR}^{2}$ pulse, the electron pulse can be compressed both, spectrally and temporally [147].

\subsection{Summary}

The electron beam emittance as well as the pulse duration benefit from a lower voltage ratio $\Gamma$. On the other hand, the transmission and thus the electron current decreases for lower values of $\Gamma$. For the operation of a UTEM, a trade-off is necessary between minimizing the emittance in order to achieve high spatial and temporal resolution, and the required electron current to obtain images within reasonable integration times. In case electrons emitted from the tip shaft are able to pass the apertures in the gun, both, emittance and pulse duration are strongly deteriorated.

A reduction of the electrons initial kinetic energy offers the possibility to reduce the emittance by one order of magnitude and the pulse duration by at least a factor of two. Such a reduction can be achieved by adjusting the laser photon energy and emitter work function relative to one another.

Within a UTEM, a reduction of the electron energy spread can be obtained after the generation of electrons employing an omega filter for energy selection. Alternatively, utilizing a radio frequency compressor, the pulse duration can be decreased as well.

\footnotetext{
${ }^{2}$ Near-infrared
} 


\section{Chapter 7}

\section{UTEM characterization}

In this chapter, an electron gun identical to the one used for measurements and simulations in the previous chapters is integrated into a transmission electron microscope. In the first part, the minimal achievable spot size of the electron beam and the electron current are investigated for different voltage settings at the electron gun. Thereafter, measurements of the spot diameter in imaging mode and the angle distribution in diffraction mode for different settings of the TEM illumination system are performed. These measurements allow to determine beam parameters such as the emittance and brightness at the specimen. The last part is addressed to show beam patterns upon illumination of the tip apex and tip shaft.

Since the alignment of the microscope is rather involved, and realignment is necessary whenever the electron gun emission characteristics are changed excessively, only selective properties are tested. Except for the last section, all of the data is recorded by illuminating the tip apex. For all measurements, the acceleration voltage of the TEM is set to $120 \mathrm{kV}$, which states the voltage applied to the tip emitter. Other voltages (e.g. extractor, suppressor voltage) are specified relative to this value.

The measurements in this chapter have been performed in close collaboration with Armin Feist and Sascha Schäfer. 
A

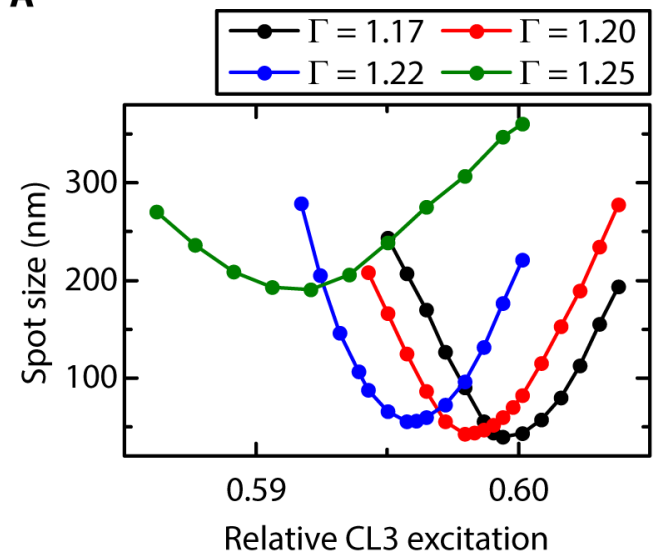

B

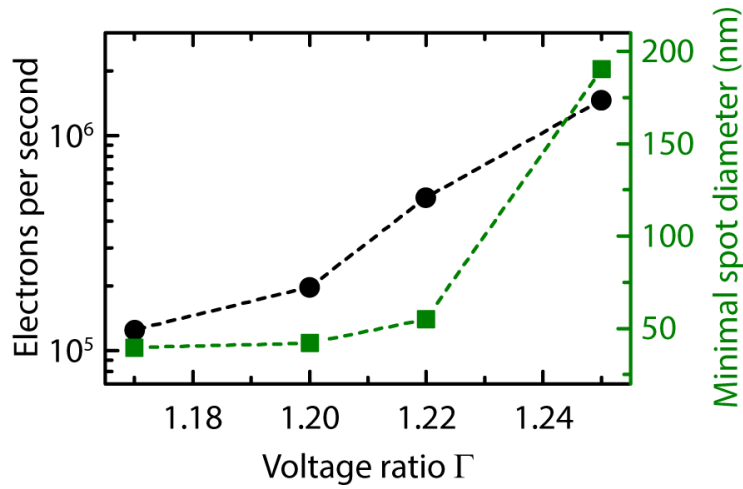

Figure 7.1: Spot diameter and electron yield for illumination of the tip apex with $400 \mathrm{~nm}$ laser light $(8.8 \mathrm{~nJ}, 30 \mathrm{~cm}$ focal length). A: Electron beam spot size (FWHM) as a function of CL3 lens (cf. Fig. 2.12) excitation for different values of $\Gamma$. B: Minimal spot size (green) and electron current in electrons per second (black) as a function of $\Gamma$.

\subsection{Electron beam $\Gamma$-dependence}

By increasing the voltage ratio $\Gamma$ at the electron gun under illumination of the tip apex, the emittance rapidly increases for $\Gamma$-values close to the field reversal (cf. Sec.6.2). This effect is a result of electrons emitted from the tip shaft being deflected through the aperture of the electron gun in addition to electrons originating from the tip apex (cf. Fig. 6.2C). As a result, the (virtual) source size and therefore the emittance and minimal achievable focus sizes increase. The effect can be measured within the UTEM by changing the suppressor voltage at constant tip and extractor potentials. Changing only the suppressor electrode has the advantage that almost no TEM realignment is necessary. The focus size is measured by changing the "brightness"-knob of the TEM, which adapts the CL3 lens and thus (de-)focuses the electron beam in the specimen plane (see Sec. 2.5.2). For the determination of the spot size itself, the root-mean-square in $x$ - and $y$-direction is calculated and converted to a full-width-half-maximum (FWHM) value of a corresponding Gaussian distribution. The gained spot sizes of both directions are subsequently averaged with a quadratic mean. In Fig. 7.1A, the results are plotted for $\Gamma$-values close to the field reversal. For this measurement, the tip voltage is set to $120 \mathrm{kV}$. Relative to this voltage, the extractor is fixed to $230 \mathrm{~V}$ and the suppressor electrode is varied between $-250 \mathrm{~V}$ and $-220 \mathrm{~V}$. The pulse energy of the $400 \mathrm{~nm}$ laser light is $8.8 \mathrm{~nJ}$. 
As predicted, the minimal spot size of the electron beam increases for rising $\Gamma$, with a strongest increase once shaft electrons dominate (cf. green and blue curves in Fig. 7.1A). The green curve in Fig. 7.1A is very close to the field reversal point, at which electron is suppressed (see Fig. 3.3 and appendent text). For a suppressor voltage of $-260 \mathrm{~V}$, corresponding to $\Gamma=1.27$, no electron emission occurred. In Fig. 7.1B, the minimal spot size is plotted as a function of $\Gamma$ (green curve) and the large increase for high $\Gamma$ values is nicely visible. In addition to the spot size, the electron current in electrons per second is shown in Fig. 7.1B as well (black curve). From $\Gamma=1.17$ to $\Gamma=1.25$, the electron current increases by more than one order of magnitude. Even though the minimal spot size increases as well, the large- $\Gamma$ regime is still useful if high resolution is not required, e.g. for coarse calibration in a low magnification, high current mode. It should be noted that in addition to the spot size, the pulse duration for high- $\Gamma$ operation of the electron beam is larger, as well (cf. Sec.6.3).

\subsection{Electron beam properties}

In order to characterize and compare the capabilities of the ultrafast electron microscope, beam characteristics such as the (transverse) emittance and brightness are measured and calculated in this section. The emittance describes the focusability of the electron beam, whereas the brightness is a combination of total electron current and emittance (see 2.4). For the calculation of the emittance, the position and momentum distributions of the electron beam are required (cf. Eq. 2.18). At the focus of the electron beam, the crosscorrelation term in Eq. 2.18 vanishes, and only the RMS-values of the spatial and angular (momentum) distribution are retained (cf. Eq. 2.21): $\varepsilon=\beta \gamma \sigma_{x} \sigma_{\alpha}$.

The spatial distribution is, again, received by averaging the RMS of the intensity profile in the two orthogonal directions. By switching the imaging system between diffraction and imaging mode, both angular and spatial distributions are obtained. The angular distribution $\left(\sigma_{\alpha}\right)$ is calculated in analogy to the spatial distribution by the RMS of the intensity profile. In order to extract a value for the convergence angle $(\alpha)$, the fullwidth-at-tenth-maximum (FWTM) is used and calculated from the standard deviation ${ }^{1}$, assuming a Gaussian profile. In Fig. 7.2A, an exemplary image of the minimal spot size is shown, and the corresponding picture in diffraction mode is depicted in Fig. 7.2B.

\footnotetext{
${ }^{1} \mathrm{FWTM}=2 \sqrt{2 \ln (10)} \cdot \sigma \approx 4.29 \cdot \sigma$
} 

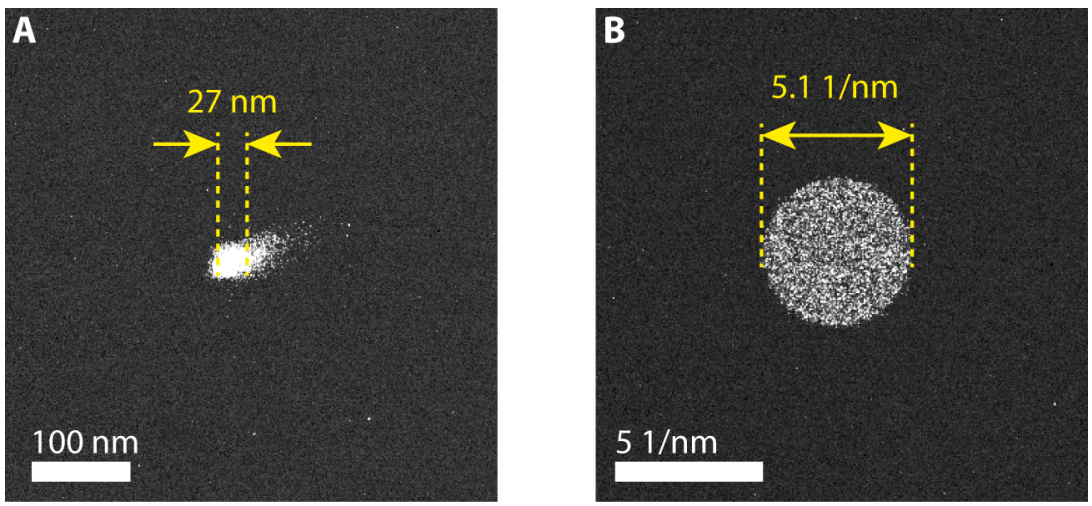

Figure 7.2: Electron beam at minimal spot size in imaging (A) and diffraction (B) mode. Illumination system settings: Spot 5, Alpha 3, CLA $3(40 \mu \mathrm{m})$.

Further results are shown in Tab.7.1. Here, the spot selector, alpha selector and condenser lens aperture (CLA) are systematically varied. Their influence on the beam shaping process is described in Sec. 2.5.2. In the first part (Tab. 7.1A), the spot selector is varied. A high spot value corresponds to a small source size, since the electron beam is defocused at the position of the CLA and subsequently blocked. As expected, the minimal achievable spot size for Spot 1 is larger than for Spot 5 (hence the name). On the other hand, the convergence angle stays constant, since the settings after the CLA are not influenced by the spot selector. In consequence of the decreasing focus size, the emittance decreases as well from a value of $145 \mathrm{~nm} \operatorname{mrad}$ (Spot 1) to $86 \mathrm{nmmrad}$ (Spot 5). For a larger spot selector setting, the electron current drops as a direct effect of blocking the electrons. However, the combination of electron current and emittance, the brightness, stays almost constant at a mean value of $6.4 \cdot 10^{4} \mathrm{~A} /\left(\mathrm{m}^{2} \mathrm{sr}\right)$.

The effect of the alpha selector (convergence angle selector) is shown in Tab.7.1B. The convergence angle can be raised from $2.5 \mathrm{mrad}$ to $6 \mathrm{mrad}$ for Spot 5 and CLA 2 (100 $\mu \mathrm{m})$. For an increasing convergence angles, the minimal beam diameter shrinks. As a result, the emittance remains constant upon variation of the alpha selector. For the used Spot-selector and CLA, a mean of $27 \mathrm{~nm}$ mrad is accomplished. By changing the convergence angle, no further electrons are cut away. Values for the brightness range from $1.74 \cdot 10^{5} \mathrm{~A} /\left(\mathrm{m}^{2} \mathrm{sr}\right)$ to $1.16 \cdot 10^{5} \mathrm{~A} /\left(\mathrm{m}^{2} \mathrm{sr}\right)$.

The third possibility to form the electron beam is the condenser lens aperture (CLA). Using Spot 1 and Alpha 3, the CLA is varied in Tab. 7.1C. The main purpose of the CLA is to trim the electron beam in order to obtain a smaller source size. However, also the convergence angle is influenced (see Sec. 2.5.2). This is reflected by the values shown 


\begin{tabular}{|c|c|c|c|c|c|c|c|c|c|c|}
\hline & $\begin{array}{c}\widetilde{0} \\
\text { की }\end{array}$ & $\frac{\widetilde{\pi}}{\stackrel{\vec{Z}}{\vec{Z}}}$ & $\underset{ت}{J}$ & 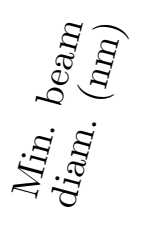 & 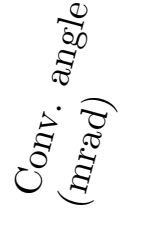 & 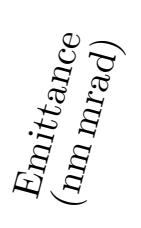 & 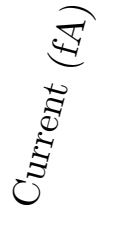 & 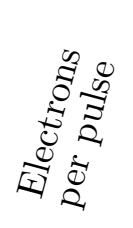 & 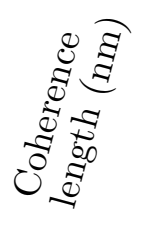 & 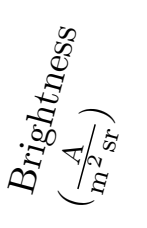 \\
\hline \multirow[t]{3}{*}{ A } & 1 & 3 & 1 & 35.1 & 11.6 & 145 & 119 & 3.0 & 0.09 & 72000 \\
\hline & 3 & 3 & 1 & 24.3 & 11.6 & 100 & 48 & 1.2 & 0.09 & 61000 \\
\hline & 5 & 3 & 1 & 20.9 & 11.5 & 86 & 35 & 0.9 & 0.09 & 60000 \\
\hline \multirow[t]{3}{*}{ B } & 5 & 1 & 2 & 31.8 & 2.5 & 25 & 8 & 0.2 & 0.49 & 174000 \\
\hline & 5 & 2 & 2 & 18.1 & 4.2 & 26 & 7.3 & 0.2 & 0.27 & 142000 \\
\hline & 5 & 3 & 2 & 14.2 & 6.0 & 30 & 8.2 & 0.2 & 0.18 & 116000 \\
\hline \multirow[t]{3}{*}{ C } & 1 & 3 & 1 & 35.1 & 11.6 & 145 & 119 & 3.0 & 0.09 & 72000 \\
\hline & 1 & 3 & 2 & 23.6 & 6.1 & 50 & 23 & 0.6 & 0.18 & 119000 \\
\hline & 1 & 3 & 3 & 20.4 & 2.6 & 17.5 & 4.2 & 0.1 & 0.45 & 173000 \\
\hline
\end{tabular}

TABLE 7.1: Measured and derived beam parameters for different settings of the illumination system (Spot selector, Alpha selector, CL-aperture). The convergence angle is defined as the opening half-angle, in accordance with the literature [46]. The emittance and brightness are normalized, as defined in Sec. 2.4.1 and Sec. 2.4.2, respectively. The numbers of the CLA aperture correspond to aperture sizes of 1: $200 \mu \mathrm{m}, 2: 100 \mu \mathrm{m}, 3$ : $40 \mu \mathrm{m}$.

in Tab.7.1C. The focus size, as well as the convergence angle, decrease for decreasing aperture sizes (increasing CLA-number). This leads to a large drop of the emittance from $145 \mathrm{~nm}$ to $17.5 \mathrm{~nm}$. Even though a smaller aperture results in a smaller electron beam current (119fA vs. $4.2 \mathrm{fA})$, the brightness increases from $7.2 \cdot 10^{4} \mathrm{~A} /\left(\mathrm{m}^{2} \mathrm{sr}\right)$ to $1.73 \cdot 10^{5} \mathrm{~A} /\left(\mathrm{m}^{2} \mathrm{sr}\right)$. The change in brightness can be explained by an inhomogeneous electron distribution at the CL aperture. Therefore, the electron current is not decreasing in the same way as the aperture area. Hence, even though the emittance is changing as well, the brightness is not necessarily conserved.

\subsubsection{Space charge considerations}

During the variation of the illumination system, a maximum of three electrons per pulse were detected. This maximum transmission is given for a setting of Spot 1 and CLA 1. The alpha-selector has no influence, as discussed before. For $\Gamma \approx 1$, the transmission through the electron gun from the simulations is $10^{-2}$ in the worst case (cf. Fig. 6.1A, aperture radius: $2 \mathrm{~mm}$ ). Assuming no further losses in the electron current, approximately 300 electrons per laser pulse are emitted from the tungsten tip. This is, however, the upper limit of the electron yield. 
In a previous work, a gold nanotip with was illuminated with $800 \mathrm{~nm}$ laser light at a repetition rate of $1 \mathrm{kHz}$. The experiment showed that even for several hundred electrons emitted with one laser pulse, the solid angle of emission did not increase [29, 144, 145]. It was concluded that electron emission from geometries with a sharp tip are less susceptible to space charge than planar surfaces.

Passlack et al. investigated two-photon photoemission spectra from a planar $\mathrm{Cu}(111)$ surface using $400 \mathrm{~nm}, 40 \mathrm{fs}$ laser light at a repetition rate of $1 \mathrm{kHz}$ and a focal size of $1 \mathrm{~mm}^{2}$ [148]. In order to preserve meaningful spectra, the photoemission yield has to be kept below 3000 electrons per pulse.

Mancini et al. constructed an electron gun consisting of a flat cathode illuminated by $266 \mathrm{~nm}$ laser pulses [164]. The generated electron pulses are temporally compress using a RF cavity. In their experiment, considering the RMS pulse duration (around $400 \mathrm{fs}$ ), $10^{5}$ electrons per pulse are feasible before the pulse duration cannot be efficiently compressed anymore [164].

In summary, the effect of space charge highly depends on the performed experiment. Specifically, the laser intensity, the emission area and the emitter shape are important. It is not possible to generally rule out any space charge effects. However, judging from the experiments described above, for a tip geometry space charge effects might be irrelevant for 100 electrons per pulse. The exact effect on the beam parameters is a matter of further research.

\subsubsection{Comparison to other UEMs}

A comparison with conventional (static) electron sources reveals that the achieved brightness of the UTEM is inferior to a static thermionic source by at least four orders of magnitude. Whereas the maximum measured normalized brightness in the UTEM is $1.74 \cdot 10^{5} \mathrm{~A} /\left(\mathrm{m}^{2} \mathrm{sr}\right)$, the brightness of a thermionic source is around $(2-10) \cdot 10^{9} \mathrm{~A} /\left(\mathrm{m}^{2} \mathrm{sr}\right)$ (cf. 2.1). For a field emission gun, the brightness easily exceeds $10^{12} \mathrm{~A} /\left(\mathrm{m}^{2} \mathrm{sr}\right)[39,45$, $46,73]$.

In order to compare pulsed electron sources among each other, as well as with static electron sources, it is reasonable to introduce a peak brightness. The peak brightness takes into account the duty cycle of an electron source. Considering the very small duty 
cycle of the UTEM $\left(\approx 10^{-7}\right)^{2}$, the peak brightness of the UTEM setup calculates to $1.74 \cdot 10^{12} \mathrm{~A} /\left(\mathrm{m}^{2} \mathrm{sr}\right)$, which is comparable to the brightness of a field emission gun.

The low energy electron gun of Merano et al. has a (normalized) brightness of $2.3 \cdot 10^{8}$ $\mathrm{A} /\left(\mathrm{m}^{2} \mathrm{sr}\right)$, which is three orders of magnitude higher than the measured value of the UTEM. However, taking into account that the laser repetition rate is over 300 times higher and the laser pulse duration is about 5 times larger compared to the UTEM setup, the peak brightness is similar in both experiments. The emittance for their setup can be calculated using the brightness and the electron current of $24 \mathrm{nA}$ to $1.14 \mu \mathrm{m} \mathrm{mrad}$. Hence, the emittance in the UTEM setup is almost two orders of magnitude better than the emittance of Merano et al..

In a theoretical work, Dowell et al. investigated the photoelectric emittance for metal cathodes [49]. With $\Phi$ as the work function and $\hbar \omega$ the photon energy, the derived normalized emittance reads

$$
\varepsilon_{n}=\sigma_{x} \sqrt{\frac{\hbar \omega-\Phi}{3 m c^{2}}}
$$

With the values of Merano et al. $(\Delta E=0.3 \mathrm{eV}, 2.5 \mu \mathrm{m}$ spot size $)$, this results in an emittance of $1.11 \mu \mathrm{m}$ mrad. The value is in very good agreement with the one calculated from the brightness. Therefore, the formula can be used to extract an expected emittance from other setups using flat photocathodes.

The group of Ahmed H. Zewail has realized a different ultrafast electron microscope with a pulsed photocathode $\left(\mathrm{LaB}_{6}\right.$ crystal) [165-168]. With the used wavelength of $343 \mathrm{~nm}$, a spot size of $30 \mu \mathrm{m}$ (FWHM) and using the above stated formula, the emittance can be calculated to $10 \mu \mathrm{m}$ mrad. This value is again significantly larger than the measured emittance in the UTEM experiment. With about one electron per pulse and a repetition rate of $80 \mathrm{MHz}$, the (normalized) brightness can be calculated to $1.6 \cdot 10^{3} \mathrm{~A} /\left(\mathrm{m}^{2} \mathrm{sr}\right)[168]$. Furthermore, taking into account the duty cycle (120 fs pulse duration), the value of the peak brightness is $1.6 \cdot 10^{8} \mathrm{~A} /\left(\mathrm{m}^{2} \mathrm{sr}\right)$, which is significantly lower than in the case of Merano et al. or the UTEM.

For single-shot experiments, at least $10^{6}$ electrons per pulse are necessary. However, due to space charge effects, the emittance will become large. Musumeci et al. prevent this by employing a radio frequency $(\mathrm{RF})$ photogun, where electrons are accelerated with more

\footnotetext{
${ }^{2}$ For a repetition rate of $250 \mathrm{kHz}$ and a pulse duration of $400 \mathrm{fs}$.
} 
than $75 \mathrm{MV} / \mathrm{m}$ to energies of several $\mathrm{MeV}$. A solenoid and a pinhole behind the gun are used to filter electrons and reach an emittance of $75 \mu \mathrm{mmrad}$. In another single-shot setup, Campbell et al. are working with $10^{9}$ electrons per pulse [169]. However, the pulse duration in their setup is on the order of $12 \mathrm{~ns}$. Using $211 \mathrm{~nm}$ laser light focused onto a tantalum disk with a focus size of $350 \mu \mathrm{m}$ (FWHM) gives an emittance of $153 \mu \mathrm{m} \mathrm{mrad}$ according to the formula of Dowell et al. [49]. This is a best case scenario, since this formula is not valid when space charge effects become relevant. The calculation of a peak brightness seems unsuited for single shot experiments, since there is no repetition and, hence, no duty cycle.

\subsection{Electron beam pattern within the TEM}

In the previous chapter, emission patterns from the electron gun assembly were shown for different voltage settings and laser focus positions on the tip emitter. In Fig. 7.3A and $\mathrm{B}$, two patterns of the electron beam are visible recorded with the UTEM setup. Both show the minimal achievable spot size for the corresponding settings. Whereas in Fig. 7.3A the tip apex is illuminated, Fig. 7.3B shows the pattern for a laser focus position $70 \mu \mathrm{m}$ away from the apex. A large difference in the spot size is clearly visible, as well as a different shape of the pattern itself. The electron beam for apex illumination can be focused to a spot of $13.5 \mathrm{~nm} \times 15.3 \mathrm{~nm}(\mathrm{FWHM})$. However, the electrons from the tip shaft form a ring pattern with a minimal diameter of $7 \mu \mathrm{m}$. Fig. 7.3C-E depict the respective profiles as indicated in Fig. 7.3A and B. The difference between the two signals can be explained in accordance to the previous chapter. For shaft emission, a dark center is observable, which corresponds to the shadowing of electron trajectories by the tip itself (see Sec.5.2). The source size in case of shaft illumination is mainly given by the laser focus size, which is on the order of $20 \mu \mathrm{m}$. For the case of apex emission, field enhancement and the curved surface of the apex itself drastically reduce the virtual source size, and thus allow for better focusing of the electron beam.

The minimal focus size achievable for this TEM is approximately $0.5 \mathrm{~nm}$ (for the applied $120 \mathrm{kV}$ acceleration voltage), considering spherical aberration of the objective lens and diffraction at the final aperture. The value is obtained by the intersection of the spot 

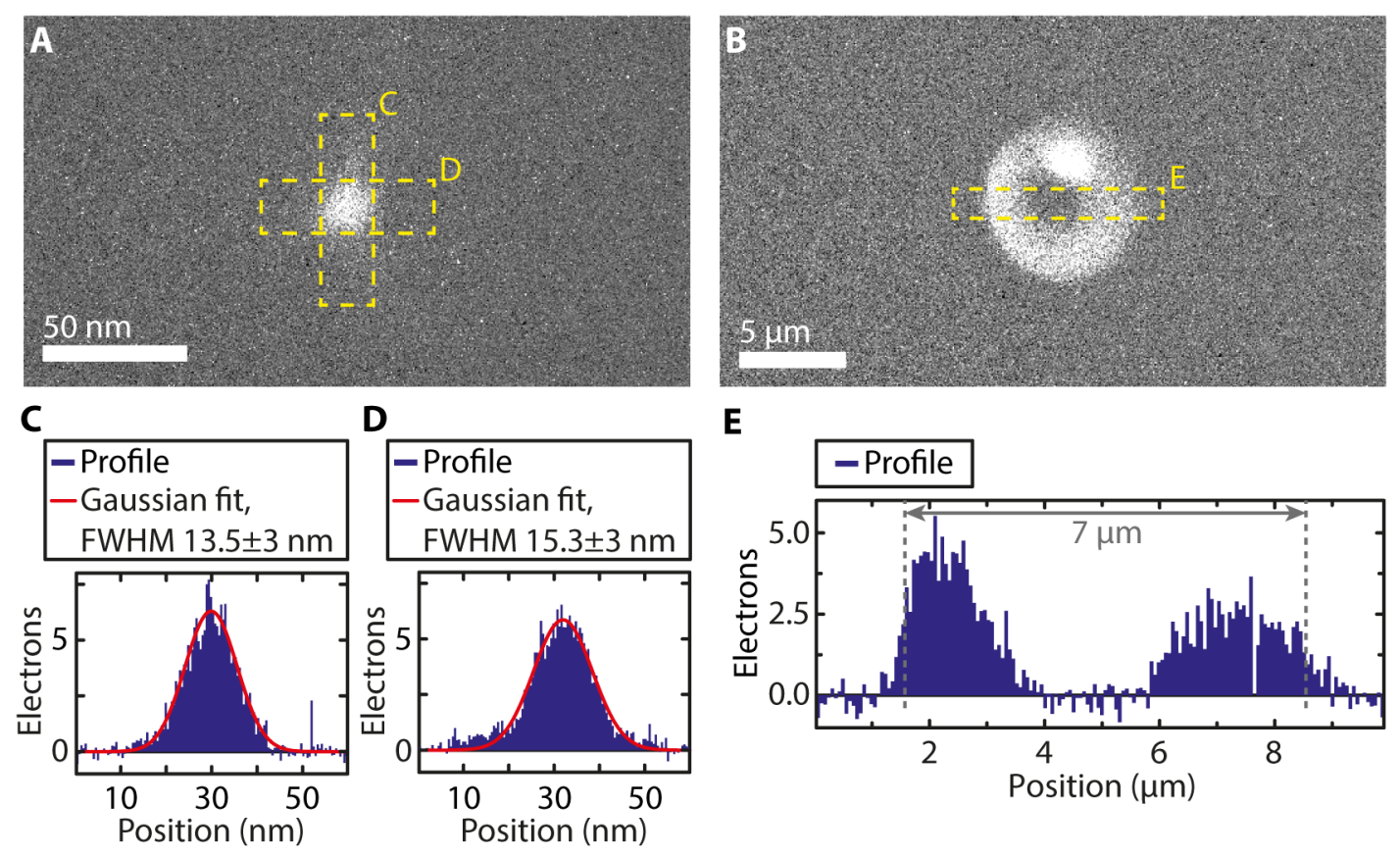

Figure 7.3: Electron beam pattern of the UTEM under illumination of the apex (A) and shaft (B). C-E: Respective cross sections of the beam profiles.

diameter dependence on the convergence angle for the two criteria. With a given spherical aberration coefficient ${ }^{3}$ of $C_{s}=1.4 \mathrm{~mm}$, the minimal spot diameter due to spherical aberrations is calculated by $[46,133]$ :

$$
d_{s}=0.5 C_{s} \alpha^{3}
$$

The diffraction limited spot diameter is given by the Rayleigh criterion [46]:

$$
d_{d}=1.22 \frac{\lambda}{\alpha}
$$

The combination of both yields an estimate of the resolution limit, even though a Gaussian distribution of the electron beam is assumed [46]. It shows that the achieved spot diameters of the experiment are neither diffraction-limited nor limited due to the spherical aberration of the objective lens. However, spherical aberrations can also occur at any other lens within the TEM column. In static TEM mode, the largest impact stems from the objective lens, hence, it is the only lens the manufacturer of the TEM states aberration coefficients for. In the UTEM mode, aberrations can also be expected at the condenser lenses (CL1-3) or even the focus lens within the electron gun assembly, since

\footnotetext{
${ }^{3}$ Value taken from the JEOL user manual.
} 
the angle of collected electrons is larger than in the conventional operation, in addition to a broader initial kinetic energy spread.

In conclusion, the minimal spot size in the UTEM is only achievable for illumination of the tip apex at electron gun voltages significantly below a $\Gamma$-value of 1.2 , in accordance with measurements and simulations of the previous chapters. 


\section{Chapter 8}

\section{Conclusions}

\subsection{Summary}

In this thesis, an electron microscope gun assembly was characterized regarding the achievement of optimum working conditions as a pulsed electron source. The findings were corroborated with numerical FEM simulations. The electron gun geometry was incorporated into a fully functional TEM, and beam parameters such as the emittance were quantified.

The main findings for optimal working conditions concerning laser-illumination and voltage settings of the gun geometry are found as the following:

- In order minimize the emittance and, thus, to achieve the smallest possible focus spot of the electron beam, electrons have to stem from the apex region of the tip emitter. The presence of a ring or arc shape indicates that either the laser is not focused onto the tip apex but the tip shaft, or the voltage ratio $\Gamma$ is above the optimum for high-magnification mode, with shaft electrons dominating the current.

- Increasing the electron gun voltage ratio $\Gamma$ (see Eq. 3.7 for definition) leads to an increase of the overall electron number on the detector and to an increase of the transverse beam emittance. Thus, a trade-off has to be made between the electron count rate, setting the recording time for capturing a single image, and the minimal emittance for high spatial resolution. 
- The simulations show that the pulse duration is only slightly affected by the voltage ratio $\Gamma$, unless shaft electrons deteriorate the beam parameters in terms of pulse duration and emittance.

- A lower excess energy, due to a better adaptation of the laser photon energy to the work function of the emitter, results in a decrease of the emittance. However, the improvement is less than one order of magnitude, and the pulse duration is only reduced by a factor of two. The effort of reducing the excess energy of the photoelectrons, e.g., by using a tunable excitation frequency from an OPA, seems to be a matter of future fine-tuning (regarding improvements see next section).

The numerical simulation results indicate an emittance in the range of $1-10 \mathrm{~nm} \mathrm{mrad}$ and the pulse duration was calculated to have an upper limit of about 1 ps. However, in the UTEM setup, an additional focus electrode is implemented in the electron gun assembly. Due to complexity, this electrode was not taken into account in the prototype chamber and in the numerical simulations. Since this electrode induces an additional fast acceleration (typical applied voltage is $6-9 \mathrm{kV}$, distance from extractor is about $20 \mathrm{~mm}$ ), the pulse duration in the UTEM setup is expected to be in the range of $100 \mathrm{fs}$ (standard deviation, cf. Ref. [37]).

In the experiments conducted with the UTEM, an emittance of $145 \mathrm{~nm}$ mrad was measured for the largest aperture size. Naturally, by introducing smaller apertures, the emittance can be decreased. The lowest measured value of the emittance was $25 \mathrm{~nm}$ mrad. Without apertures installed in the UTEM, the minimal spot diameter is $35.1 \mathrm{~nm}$ (cf. Tab. 7.1). The measured maximum (normalized) brightness is $1.74 \cdot 10^{5} \mathrm{~A} /\left(\mathrm{m}^{2} \mathrm{sr}\right)$. Considering the duty cycle of the $\mathrm{UTEM}^{1}$, this value corresponds to a peak brightness of $1.74 \cdot 10^{12} \mathrm{~A} /\left(\mathrm{m}^{2} \mathrm{sr}\right)$, which is comparable to the brightness of a field emission gun. It has to be noted that these are preliminary values, since the UTEM is still under development and subject to continuous improvement. In terms of peak current, the UTEM delivers approx. $10^{5}$ electrons per second. For a conventional field emission gun, assuming a dc-current on the detector of $100 \mathrm{pA}$, the same duty cycle would correspond to about 10 electrons per second, rendering image acquisition impossible.

Electrons stemming from the shaft area of the emitter are undesired in order to receive optimum conditions. Shaft-emitted electrons start to contribute to the detected current

\footnotetext{
${ }^{1}$ The duty cycle calculates to $10^{-7}$ for a repetition rate of $250 \mathrm{kHz}$ and a pulse duration of $400 \mathrm{fs}$.
} 
close to the field reversal at the apex of the emitter, which occurs for very high values of $\Gamma$ even if the laser is illuminating the tip apex. A characteristic feature of shaft electrons is a drastic increase of the minimal achievable focal spot and a simultaneous increase of the total electron current upon increasing $\Gamma$ (cf. Fig. 7.1). This mode of operation is particularly useful to enable a short integration time at a reduced beam quality, e.g., as a low magnification, high electron count mode. Such a source mode could be used in order to either find the region of interest within the specimen, or for large scale overview images with lower resolution ( $\mu \mathrm{m}-\mathrm{scale})$.

\subsection{Outlook}

This thesis elaborates the impact of voltage settings and illumination conditions onto spatial and temporal beam characteristics for an ultrafast electron gun. Research continuing this work can be divided into short term goals using the existing setup with minor modifications, and general, more involved ideas.

As a direct continuation of this thesis, electron pulse durations should be systematically measured within the UTEM for a variety of parameters. The method of choice for characterizing electron pulses is based on the photon-induced inelastic near-field scattering [170-173]. Here, the energy spectrum of the electron pulse is modified upon passing a nanostructure, which is illuminated by an intense optical pulse. The modification depends on the temporal overlap between the electron and laser pulse, and allows to determine the pulse duration of the electron pulse [37, 171]. In a first experiment using the UTEM, a value of $340 \mathrm{fs}$ was measured [37]. In order to find an optimum configuration for high temporal and high spatial resolution, measuring the pulse duration as a function of electron gun parameters is required. Specifically, the dependence on the voltage ratio $\Gamma$ and apertures (CLA) in the UTEM is expected to have the largest impact.

In order to increase the total number of electrons and to be able to adjust the pulse duration of the electron pulse more easily by tuning the laser pulse duration, the electron emission process can be changed from pure two-photon photoemission to a thermally assisted one-photon photoemission process $[21,174]$. In this scenario, a $\mathrm{ZrO}_{2}$-tungsten emitter is employed and heated above $1500 \mathrm{~K}$ in order to obtain a $\mathrm{ZrO}_{2}$-layer covering the tungsten tip. The reduced work function $(\Phi=2.7 \mathrm{eV})$ in combination with a heated tip 
results in single-photon photoemission for $400 \mathrm{~nm}$ laser light. By applying a high bias voltage between the tip and extractor electrode, the work function can be selectively reduced at the tip apex (Schottky effect) to further improve the localization of the emission process [175]. A side-effect of this approach is, however, that the surface at the tip apex is modified due to the applied heat and voltage. The result is a flat frontfacet, the size of which depends on the initial apex diameter [39, 176]. Most of the emitted electrons are expected to originate from the front-facet due to the very localized reduction of the work function along different crystallographic orientations. For the thermally assisted single-photon approach, the emittance was measured very recently in our group by Armin Feist to be as low as $4 \mathrm{~nm}$ mrad. Furthermore, due to the linear response of the effect and the fact that no high intensities are necessary for single-photon photoemission, the duration of the electron pulse can be tuned more easily than in the case of nonlinear photoemission. It will be up to further research whether the thermallyfield-assisted single-photon emission approach is superior to a two-photon photoemission process.

A second approach for one-photon photoemission is to use the third harmonic of a titanium sapphire laser, which has a photon energy of $4.65 \mathrm{eV}$. In combination with an applied bias voltage and a single-crystalline tungsten wire in, e.g., $\langle 100\rangle$ orientation (work function $4.6 \mathrm{eV}$ ), the energy of a single photon is sufficient to overcome the workfunction. The main advantage of this single-photon approach is the low excess energy for this combination and - in comparison to a heated tip - no expected structural change of the emitter. However, one complicating issue could arise from the electron gun material. The stainless steel used for the electrodes within the TEM-gun has a work function of only $4.5 \mathrm{eV}$. Therefore, unwanted electron emission from gun parts illuminated by stray light might occur and interfere with the desired emission process.

Aside from single-photon emission, interesting questions arise in case of nonlinear photoemission, as well. As a direct continuation of this work, two-photon photoemission employing an optical parametric amplifier (OPA) should be investigated. Utilizing such an OPA allows for tuning the laser wavelength in such a way, that the excess energy of emitted electrons is minimized. A higher nonlinearity might be beneficial, e.g., a threephoton process, since electrons emitted from the tip apex will be dominant compared to electrons stemming from the tip shaft. Furthermore, the localization of the emission at the front side of the apex will be improved, which might further reduce the emittance. 
Further, more involved investigations include $\mathrm{THz}$ streaking and gating of the electron pulse upon emission (see, e.g., Ref. [147, 162, 163]) within the UTEM, the dependence of the tip apex diameter on the beam parameters, or employing a gold nanotip with a grating etched into the surface in order to obtain localized electron emission due to excited plasmons. The latter has the advantage of providing better absorption of the incident laser light as well as better heat transport due to the illumination of a larger surface area. Furthermore, an increase in the electron yield was recently reported [177]. Ongoing research concerning this topic is done in our group by Benjamin Schröder and already achieved promising results [178].

Generally, improvements of the setup could be made by implementing either a radiofrequency compressor or an $\Omega$-filter in order to reduce the electron pulse duration (see Sec.6.3). With the utilization of an $\Omega$-filter, reducing the energy spread of the electron pulse is possible, however, at the cost of sacrificing the overall number of electrons, which will increase the integration time of the camera in order to obtain a picture. A combination of adjusting the initial kinetic energy of photoemitted electrons (low excess energy) and overcoming the drop of the electron yield (high repetition rate) could be achieved by an optical parametric oscillator.

To conclude, this work demonstrates the advantages of a tip-based ultrafast electron gun for time-resolved electron microscopy. The approach allows for tailoring of the photoelectron emission site in order to achieve optimum working conditions regarding a high magnification or a large count rate, respectively. With further effort, the tipbased UTEM approach also bears the potential to perform single-shot experiments, allowing for extended application in materials science. Whereas the UTEM in its current state already allows for unprecedented time-resolved measurements with high spatial resolution, improving the UTEM-performance is a subject of intense ongoing research. Consequently, the emerging field of ultrafast dynamics at inhomogeneities, defects or interfaces finally becomes accessible via ultrafast electron microscopy. 



\title{
Appendix A
}

\section{Abbreviations and symbols}

\author{
Abbreviations \\ $\mathrm{BBO}$ beta barium borate \\ BK7 borosilicate crown glass \\ Chap. chapter \\ CBED convergent beam diffraction \\ CCD charge-coupled device \\ CL condenser lens \\ CLA condenser lens aperture \\ CM condenser mini-lens \\ CMOS complementary metal-oxide-semiconductor \\ DC direct current \\ $e^{-} \quad$ electron \\ Eq. equation \\ ext extractor \\ FEM finite element method \\ Fig. figure \\ FN Fowler-Nordheim \\ fps frames per second \\ FWHM full-width-at-half-maximum \\ FWTM full-width-at-tenth-maximum \\ $\mathrm{KOH}$ potassium hydroxide
}


$\mathrm{LaB}_{6} \quad$ lanthanum hexaboride

LEED low-energy electron diffraction

MCP micro-channel-plate

ND neutral density

NIR near-infrared

OPA optical parametric amplifier

PS phosphorous screen

$\mathrm{RF} \quad$ radio frequency

RMS root-mean-square

Sec. section

SHG second harmonic generation

STEM scanning transmission electron microscope

STM scanning tunneling microscope

sup suppressor

TEM transmission electron microscope

UHV ultra-high vacuum

UTEM ultrafast transmission electron microscope

$\mathrm{Zr} \quad$ zirconium

$\mathrm{ZrH}_{2} \quad$ zirconium hydride

$\mathrm{Zr}-\mathrm{O}$ zirconium oxide

$\mathrm{ZrO}_{2} \quad$ zirconium dioxide

\section{Symbols}

$\begin{array}{ll}c & \text { speed of light } \\ e & \text { elementary charge } \\ \varepsilon_{0} & \text { vacuum permittivity } \\ \hbar, h & \text { (reduced) Planck constant } \\ k_{B} & \text { Boltzmann constant } \\ m_{e} & \text { electron rest mass } \\ A & \text { area } \\ B & \text { brightness } \\ C_{c} & \text { chromatic aberration coefficient }\end{array}$




\begin{tabular}{|c|c|}
\hline$C_{s}$ & spherical aberration coefficient \\
\hline$d$ & distance; length \\
\hline$d_{c}$ & coherence length \\
\hline$E$ & energy \\
\hline$f_{\text {rep }}$ & repetition rate \\
\hline$F$ & electric field \\
\hline$G$ & probability density function \\
\hline$I$ & intensity \\
\hline$J$ & current density \\
\hline$k$ & geometry factor \\
\hline$k_{\text {laser }}$ & wave vector \\
\hline$L$ & distance \\
\hline$n$ & refractive index \\
\hline$N$ & number of electrons \\
\hline$r$ & radius \\
\hline$s$ & length \\
\hline$t$ & time \\
\hline$T$ & temperature \\
\hline$U$ & potential \\
\hline$U_{p}$ & ponderomotive potential \\
\hline$v$ & velocity \\
\hline$x$ & distance \\
\hline$\alpha$ & angle \\
\hline$\beta$ & field enhancement factor; relativistic factor \\
\hline$\gamma$ & Keldysh parameter; relativistic factor \\
\hline$\Gamma$ & voltage ratio (see Eq. 3.7 for definition) \\
\hline$\varepsilon$ & emittance \\
\hline$\eta$ & intercept \\
\hline$\theta$ & half-angle \\
\hline$\Theta$ & Heaviside function \\
\hline$\lambda$ & wavelength \\
\hline$\mu$ & mean \\
\hline$\xi$ & slope \\
\hline
\end{tabular}


standard deviation

$\Phi \quad$ work-function

$\omega \quad$ angular frequency

$\Omega \quad$ solid angle

$(h k l),\langle h k l\rangle \quad$ miller indices

$\mathbf{n}$

surface normal vector

$\mathbf{r}$

coordinate vector

$x, y, z$

Cartesian space coordinates

$r, \varphi, z$

cylindrical space coordinates

$p_{x}, p_{y}, p_{z}$

momentum coordinates 


\section{Bibliography}

[1] S. L. Johnson, E. Vorobeva, P. Beaud, C. J. Milne, and G. Ingold. Full Reconstruction of a Crystal Unit Cell Structure during Coherent Femtosecond Motion. Phys. Rev. Lett., 103(20):205501, 2009. Cited on page 1.

[2] C. Rischel, A. Rousse, I. Uschmann, P.-A. Albouy, J.-P. Geindre, P. Audebert, J.-C. Gauthier, E. Fröster, J.-L. Martin, and A. Antonetti. Femtosecond timeresolved X-ray diffraction from laser-heated organic films. Nature, 390(6659):490492, 1997. Cited on page 1.

[3] A. Rousse, C. Rischel, and J.-C. Gauthier. Femtosecond x-ray crystallography. Rev. Mod. Phys., 73(1):17-31, 2001. Cited on page 1.

[4] S. L. Johnson, P. Beaud, C. J. Milne, F. S. Krasniqi, E. S. Zijlstra, M. E. Garcia, M. Kaiser, D. Grolimund, R. Abela, and G. Ingold. Nanoscale DepthResolved Coherent Femtosecond Motion in Laser-Excited Bismuth. Phys. Rev. Lett., 100(15):155501, 2008. Cited on page 1.

[5] K. Sokolowski-Tinten, C. Blome, J. Blums, A. Cavalleri, C. Dietrich, A. Tarasevitch, I. Uschmann, E. Förster, M. Kammler, M. Horn-von Hoegen, and D. von der Linde. Femtosecond X-ray measurement of coherent lattice vibrations near the Lindemann stability limit. Nature, 422(6929):287-289, 2003. Cited on page 1.

[6] G. Sciaini and R. J. D. Miller. Femtosecond electron diffraction: heralding the era of atomically resolved dynamics. Reports Prog. Phys., 74(9):096101, 2011. Cited on pages 1,20 and 27.

[7] B. J. Siwick, J. R. Dwyer, R. E. Jordan, and R. J. D. Miller. An atomic-level view of melting using femtosecond electron diffraction. Science, 302(5649):1382-1385, 2003. Cited on pages 1,19 and 20. 
[8] M. Eichberger, H. Schäfer, M. Krumova, M. Beyer, J. Demsar, H. Berger, G. Moriena, G. Sciaini, and R. J. D. Miller. Snapshots of cooperative atomic motions in the optical suppression of charge density waves. Nature, 468(7325):799-802, 2010. Cited on page 1.

[9] P. Baum, D.-S. Yang, and A. H. Zewail. 4D visualization of transitional structures in phase transformations by electron diffraction. Science, 318(5851):788792, 2007. Cited on page 1.

[10] T. LaGrange, G. H. Campbell, B. W. Reed, M. Taheri, J. B. Pesavento, J. S. Kim, and N. D. Browning. Nanosecond time-resolved investigations using the in situ of dynamic transmission electron microscope (DTEM). Ultramicroscopy, 108(11):1441-1449, 2008. Cited on page 1.

[11] F. Carbone, O.-H. Kwon, and A. H. Zewail. Dynamics of Chemical Bonding Mapped by Energy-Resolved 4D Electron Microscopy. Science, 325(5937):181184, 2009. Cited on page 1.

[12] D. J. Flannigan and A. H. Zewail. 4D Electron Microscopy: Principles and Applications. Acc. Chem. Res., 45(10):1828-1839, 2012. Cited on pages 1, 19 and 20.

[13] M. Gao, C. Lu, H. Jean-Ruel, Lai C. Liu, A. Marx, K. Onda, S. Koshihara, Y. Nakano, X. Shao, T. Hiramatsu, G. Saito, H. Yamochi, R. R. Cooney, G. Moriena, G. Sciaini, and R. J. D. Miller. Mapping molecular motions leading to charge delocalization with ultrabright electrons. Nature, 496(7445):343-346, 2013. Cited on page 1 .

[14] A. Hanisch-Blicharski, A. Janzen, B. Krenzer, S. Wall, F. Klasing, A. Kalus, T. Frigge, M. Kammler, and M. Horn-von Hoegen. Ultra-fast electron diffraction at surfaces: From nanoscale heat transport to driven phase transitions. Ultramicroscopy, 127:2-8, 2013. Cited on page 1.

[15] M. Eichberger, N. Erasmus, K. Haupt, G. Kassier, a. Von Flotow, J. Demsar, and H. Schwoerer. Femtosecond streaking of electron diffraction patterns to study structural dynamics in crystalline matter. Appl. Phys. Lett., 102(12), 2013. Cited on page 1. 
[16] L. Piazza, C. Ma, H. X. Yang, A. Mann, Y. Zhu, J. Q. Li, and F. Carbone. Ultrafast structural and electronic dynamics of the metallic phase in a layered manganite. Struct. Dyn., 1(1):014501, 2014. Cited on page 1.

[17] M. Gulde, S. Schweda, G. Storeck, M. Maiti, H. K. Yu, A. M. Wodtke, S. Schäfer, and C. Ropers. Ultrafast low-energy electron diffraction in transmission resolves polymer/graphene superstructure dynamics. Science, 345(6193):200-204, 2014. Cited on pages 1, 2, 19 and 38 .

[18] A. H. Zewail. Four-Dimensional Electron Microscopy. Science, 328(5975):187193, 2010. Cited on pages 1 and 6 .

[19] R. J. D. Miller. Femtosecond Crystallography with Ultrabright Electrons and Xrays: Capturing Chemistry in Action. Science, 343(6175):1108-1116, 2014. Cited on page 1 .

[20] M. Müller, A. Paarmann, and R. Ernstorfer. Femtosecond electrons probing currents and atomic structure in nanomaterials. Nat. Commun., 5:5292, 2014. Cited on page 1 .

[21] D.-S. Yang, O. F. Mohammed, and A. H. Zewail. Scanning ultrafast electron microscopy. Proc. Natl. Acad. Sci., 107(34):14993-14998, 2010. Cited on pages 1 and 101.

[22] J. C. H. Spence, U. Weierstall, and H. N. Chapman. X-ray lasers for structural and dynamic biology. Reports Prog. Phys., 75(10):102601, 2012. Cited on page 1.

[23] M. Aeschlimann, E. Hull, J. Cao, C. A. Schmuttenmaer, L. G. Jahn, Y. Gao, H. E. Elsayed-Ali, D. A. Mantell, and M. R. Scheinfein. A picosecond electron gun for surface analysis. Rev. Sci. Instrum., 66(1995):1000-1009, 1995. Cited on page 1.

[24] L. Piazza, M. Cottet, F. Carbone, D. J. Masiel, and T. LaGrange. Principles and Implementation of an Ultrafast Transmission Electron Microscope. Microsc. Microanal., 18(S2):600-601, 2012. Cited on pages 1 and 6.

[25] M. Gao, Y. Jiang, G. H. Kassier, and D. R. J. Miller. Single shot time stamping of ultrabright radio frequency compressed electron pulses. Appl. Phys. Lett., 103(3):033503, 2013. Cited on page 1. 
[26] T. van Oudheusden, E. F. de Jong, S. B. van der Geer, W. P. E. M. Op 't Root, O. J. Luiten, and B. J. Siwick. Electron source concept for single-shot sub-100 fs electron diffraction in the $100 \mathrm{keV}$ range. J. Appl. Phys., 102(9):093501, 2007. Cited on pages 2, 7, 19, 20, 27 and 53 .

[27] P. Baum. On the physics of ultrashort single-electron pulses for time-resolved microscopy and diffraction. Chem. Phys., 423:55-61, 2013. Cited on pages 2, 5, $7,20,25$ and 27.

[28] C. Ropers, D. R. Solli, C. P. Schulz, C. Lienau, and T. Elsaesser. Localized Multiphoton Emission of Femtosecond Electron Pulses from Metal Nanotips. Phys. Rev. Lett., 98(4):043907, 2007. Cited on pages 2, 15, 16 and 39.

[29] R. Bormann, M. Gulde, A. Weismann, S. V. Yalunin, and C. Ropers. TipEnhanced Strong-Field Photoemission. Phys. Rev. Lett., 105(14):147601, 2010. Cited on pages 2, 15, 16, 18, 38, 40, 52, 53 and 94 .

[30] H. Yanagisawa, C. Hafner, P. Doná, M. Klöckner, D. Leuenberger, T. Greber, M. Hengsberger, and J. Osterwalder. Optical Control of Field-Emission Sites by Femtosecond Laser Pulses. Phys. Rev. Lett., 103(25):257603, 2009. Cited on pages $2,15,64$ and 65 .

[31] B. Barwick, C. Corder, J. Strohaber, N. Chandler-Smith, C. Uiterwaal, and H. Batelaan. Laser-induced ultrafast electron emission from a field emission tip. New J. Phys., 9(5):142, 2007. Cited on pages 2, 15, 16 and 18.

[32] P. Hommelhoff, C. Kealhofer, and M. A. Kasevich. Ultrafast Electron Pulses from a Tungsten Tip Triggered by Low-Power Femtosecond Laser Pulses. Phys. Rev. Lett., 97(24):247402, 2006. Cited on pages 2, 15 and 16.

[33] P. Hommelhoff, Y. Sortais, A. Aghajani-Talesh, and M. A. Kasevich. Field Emission Tip as a Nanometer Source of Free Electron Femtosecond Pulses. Phys. Rev. Lett., 96(7):077401, 2006. Cited on pages 2 and 16.

[34] A. Paarmann, M. Gulde, M. Müller, S. Schäfer, S. Schweda, M. Maiti, C. Xu, T. Hohage, F. Schenk, C. Ropers, and R. Ernstorfer. Coherent femtosecond lowenergy single-electron pulses for time-resolved diffraction and imaging: A numerical study. J. Appl. Phys., 112(11):113109, 2012. Cited on pages 2, 38, 39 and 83. 
[35] M. Gulde. Development of an ultrafast low-energy electron diffraction setup. $\mathrm{PhD}$ thesis. Georg-August-Universität Göttingen, 2014. Cited on pages 2, 19, 38, 39, $42,52,83$ and 86 .

[36] A. H. Zewail and J. M. Thomas. 4D Electron Microscopy. Imperial College Press, 2010. Cited on pages 5 and 26.

[37] A. Feist, K. E. Echternkamp, J. Schauss, S. V. Yalunin, S. Schäfer, and C. Ropers. Quantum coherent optical phase modulation in an ultrafast transmission electron microscope. Nature, 521(7551):200-203, 2015. Cited on pages 6, 100 and 101.

[38] A. J. DeMaria, W. H. Glenn, M. J. Brienza, and M. E. Mack. Picosecond laser pulses. Proc. IEEE, 57(1):2-25, 1969. Cited on page 6.

[39] J. Orloff. Handbook of Charged Particle Optics. 2nd edition, CRC Press, 2008. Cited on pages 8, 9, 10, 11, 20, 23, 86, 94 and 102.

[40] S. Dushman. Electron Emission from Metals as a Function of Temperature. Phys. Rev., 21(6):623-636, 1923. Cited on page 8.

[41] C. Herring and M. H. Nichols. Thermionic Emission. Rev. Mod. Phys., 21(2):185270, 1949. Cited on page 8.

[42] N. W. Ashcroft and N. D. Mermin. Festkörperphysik. 2nd edition, Oldenbourg Wissenschaftsverlag, 2005. Cited on page 8 .

[43] A. Modinos. Field, Thermionic, and Secondary Electron Emission Spectroscopy. Springer, 1984. Cited on pages 8, 12 and 13.

[44] D. B. Williams and C. B. Carter. Transmission Electron Microscopy. Plenum Press, 1996. Cited on pages 8, 9 and 13.

[45] R. F. Egerton. Physical Principles of Electron Microscopy. Springer, 2005. Cited on pages $9,11,13,14,29$ and 94.

[46] D. B. Williams and C. B. Carter. Transmission Electron Microscopy. 2nd edition, Springer, 2009. Cited on pages 9, 13, 23, 25, 26, 28, 31, 87, 93, 94 and 97.

[47] M. Bakr, R. Kinjo, Y. W. Choi, M. Omer, K. Yoshida, S. Ueda, M. Takasaki, K. Ishida, N. Kimura, H. Zen, T. Sonobe, T. Kii, K. Masuda, and H. Ohgaki. Comparison of Heating Property of LaB6 and CeB6 by Back Bombardment Effect in Thermionic RF gun. J. Korean Phys. Soc., 59(5):3273-3279, 2011. Cited on page 9 . 
[48] I. Bat'ko, M. Bat'ková, K. Flachbart, V. B. Filippov, Yu. B. Paderno, N. Yu. Shicevalova, and Th. Wagner. Electrical resistivity and superconductivity of LaB6 and LuB12. J. Alloys Compd., 217(2):L1-L3, 1995. Cited on page 9.

[49] D. H. Dowell and J. F. Schmerge. Quantum efficiency and thermal emittance of metal photocathodes. Phys. Rev. Spec. Top. - Accel. Beams, 12(7):074201, 2009. Cited on pages 10, 22, 39, 95 and 96.

[50] E. L. Murphy and R. H. Good. Thermionic Emission, Field Emission, and the Transition Region. Phys. Rev., 102(6):1464-1473, 1956. Cited on page 10.

[51] W. Schottky. Über den Einfluss von Strukturwirkungen, besonders der Thomsonschen Bildkraft, auf die Elektronenemission der Metalle. Phys. Zeitschrift, 15:872-878, 1914. Cited on page 10.

[52] D. C. Bell and N. Erdman. Low Voltage Electron Microscopy. Wiley, 2013. Cited on pages 11,29 and 30 .

[53] Y. Terui, K. Tsunoda, and M. Ide. Thermal field emission cathode. U.S. Patent 5,449,968, issued Sep. 12, 1995. Cited on page 11.

[54] J. E. Wolfe. Operational experience with zirconiated T-F emitters. J. Vac. Sci. Technol., 16(6):1704, 1979. Cited on page 11.

[55] P. R. Thornton. Electron Physics in Device Microfabrication. I General Background and Scanning Systems. Ed. by L. Marton. Advances in Electronics and Electron Physics. Academic Press, 1979, pp. 271-380. Cited on page 11.

[56] L. W. Swanson and N. A. Martin. Field electron cathode stability studies: Zirconium/tungsten thermal-field cathode. J. Appl. Phys., 46(5):2029-2050, 1975. Cited on pages 11 and 12 .

[57] L. R. Danielson and L. W. Swanson. High temperature coadsorption study of zirconium and oxygen on the W(100) crystal face. Surf. Sci., 88(1):14-30, 1979. Cited on pages 11 and 12 .

[58] L. W. Swanson and D. Tuggle. Recent progress in thermal field electron source performance. Appl. Surf. Sci., 8(1-2):185-196, 1981. Cited on pages 11 and 12.

[59] S. C. Lee, Y. Irokawa, M. Inoue, and R. Shimizu. Behavior of zirconium in the $\mathrm{ZrO} / \mathrm{W}(100)$ system at high temperature, studied by ISS, AES and work-function measurements. Surf. Sci., 330(3):289-296, 1995. Cited on page 12. 
[60] H. Satoh, H. Nakane, and H. Adachi. Behavior of zirconium on $\mathrm{ZrO} / \mathrm{W}(100)$ surface. Appl. Surf. Sci., 100-101:216-221, 1996. Cited on page 12.

[61] S. C. Lee, Y. Irokawa, M. Inoue, and R. Shimizu. Surface segregation of zirconium in oxygen-processed system at high temperatures. Surf. Sci., 359(1-3):198-204, 1996. Cited on page 12.

[62] R. H. Fowler and L. Nordheim. Electron Emission in Intense Electric Fields. Proc. R. Soc. London A, 119(781):173-181, 1928. Cited on page 12.

[63] R. G. Forbes. Simple good approximations for the special elliptic functions in standard Fowler-Nordheim tunneling theory for a Schottky-Nordheim barrier. Appl. Phys. Lett., 89(11):113122, 2006. Cited on page 12.

[64] C. A. Spindt, I. Brodie, L. Humphrey, and E. R. Westerberg. Physical properties of thin-film field emission cathodes with molybdenum cones. J. Appl. Phys., 47(12):5248-5263, 1976. Cited on page 12.

[65] J. Paulini, T. Klein, and G. Simon. Thermo-field emission and the Nottingham effect. J. Phys. D, 26(8):1310-1315, 1993. Cited on page 12.

[66] R. E. Burgess, H. Kroemer, and J. M. Houston. Corrected Values of FowlerNordheim Field Emission Functions v(y) and s(y). Phys. Rev., 90(4):515, 1953. Cited on page 12 .

[67] R. Gomer. Field Emission and Field Ionization. Harvard University Press, 1961. Cited on pages 12, 13, 55 and 56.

[68] H. D. Beckey, H. Krone, and F. W. Roellgen. Comparison of tips, thin wires and sharp metal edges as emitters for field ionization mass spectrometry. J. Phys. E., 1(2):118-120, 1968. Cited on page 13.

[69] K. Skotnicová, J. Drápala, and V. Kolarík. Study of emission properties of tungsten single crystal with crystallographic orientation. 19th Int. Conf. Metall. Mater. - Met. 2010. Roznov pod Radhostem, Czech Republic, 2010, p. 5. Cited on pages 13,52 and 56 .

[70] C. E. Mendenhall and C. F. DeVoe. The Photoelectric Work Functions of the 211 and 310 Planes of Tungsten. Phys. Rev., 51(5):346-349, 1937. Cited on page 13.

[71] J. C. H. Spence. High-Resolution Electron Microscopy. 4th edition, Oxford University Press, 2013. Cited on pages 13 and 24. 
[72] R. Brydson. Aberration-Corrected Analytical Transmission Electron Microscopy. Wiley, 2011. Cited on page 13.

[73] L. Reimer and H. Kohl. Transmission Electron Microscopy. 5th edition, Springer, 2008. Cited on pages 14 and 94.

[74] Values taken from www.YPS-Ltd.com. Cited on page 14.

[75] E. Hecht. Optics. 4th edition, Addison-Wesley, 2001. Cited on page 14.

[76] A. Einstein. Über einen die Erzeugung und Verwandlung des Lichtes betreffenden heuristischen Gesichtspunkt. Ann. Phys., 322(6):132-148, 1905. Cited on page 14.

[77] M. Göppert-Mayer. Über Elementarakte mit zwei Quantensprüngen. Ann. Phys., 401(3):273-294, 1931. Cited on page 14.

[78] P. A. Franken, A. E. Hill, C. W. Peters, and G. Weinreich. Generation of Optical Harmonics. Phys. Rev. Lett., 7(4):118-119, 1961. Cited on pages 15 and 48.

[79] J. H. Bechtel, W. L. Smith, and N. Bloembergen. Four-photon photoemission from tungsten. Opt. Commun., 13(1):56-59, 1975. Cited on pages 15 and 52.

[80] J. H. Bechtel, W. L. Smith, and N. Bloembergen. Two-photon photoemission from metals induced by picosecond laser pulses. Phys. Rev. B, 15(10):4557-4563, 1977. Cited on pages 15 and 52.

[81] R. Yen, J. Liu, and N. Bloembergen. Thermally assisted multiphoton photoelectric emission from tungsten. Opt. Commun., 35(2):277-282, 1980. Cited on pages 15,52 and 53.

[82] P. Agostini, F. Fabre, G. Mainfray, G. Petite, and N. K. Rahman. Free-Free Transitions Following Six-Photon Ionization of Xenon Atoms. Phys. Rev. Lett., 42(17):1127-1130, 1979. Cited on page 15.

[83] L.-A. Lompre, A. L'Huillier, G. Mainfray, and J. Y. Fan. Electron energy measurements in multiphoton ionisation of xenon and neon. J. Phys. B, 17(23):L817L822, 1984. Cited on page 15.

[84] T. J. McIlrath, P. H. Bucksbaum, R. R. Freeman, and M. Bashkansky. Abovethreshold ionization processes in xenon and krypton. Phys. Rev. A, 35(11):46114623, 1987. Cited on page 15. 
[85] M. D. Perry, A Szoke, O. L. Landen, and E. M. Campbell. Nonresonant multiphoton ionization of noble gases: Theory and experiment. Phys. Rev. Lett., 60(13):1270-1273, 1988. Cited on page 15.

[86] S. Augst, D. D. Meyerhofer, D. Strickland, and S. L. Chint. Laser ionization of noble gases by Coulomb-barrier suppression. J. Opt. Soc. Am. B, 8(4):858, 1991. Cited on page 15 .

[87] T. Tsang, T. Srinivasan-Rao, and J. Fischer. Surface-plasmon field-enhanced multiphoton photoelectric emission from metal films. Phys. Rev. B, 43(11):8870-8878, 1991. Cited on page 15.

[88] M. Aeschlimann, C. A. Schmuttenmaer, H. E. Elsayed-Ali, R. J. D. Miller, J. Cao, Y. Gao, and D. A. Mantell. Observation of surface enhanced multiphoton photoemission from metal surfaces in the short pulse limit. J. Chem. Phys., 102(21):8606-8613, 1995. Cited on page 15.

[89] J. Kupersztych, P. Monchicourt, and M. Raynaud. Ponderomotive Acceleration of Photoelectrons in Surface-Plasmon-Assisted Multiphoton Photoelectric Emission. Phys. Rev. Lett., 86(22):5180-5183, 2001. Cited on page 15.

[90] C. Ropers, C. C. Neacsu, T. Elsaesser, M. Albrecht, M. B. Raschke, and C. Lienau. Grating-Coupling of Surface Plasmons onto Metallic Tips: A Nanoconfined Light Source. Nano Lett., 7(9):2784-2788, 2007. Cited on page 15.

[91] M. Schenk, M. Krüger, and P. Hommelhoff. Strong-Field Above-Threshold Photoemission from Sharp Metal Tips. Phys. Rev. Lett., 105(25):257601, 2010. Cited on pages 15,18 and 39 .

[92] G. Herink, D. R. Solli, M. Gulde, and C. Ropers. Field-driven photoemission from nanostructures quenches the quiver motion. Nature, 483(7388):190-193, 2012. Cited on pages 15, 16, 18, 19, 53 and 83 .

[93] C. Ropers, T. Elsaesser, G. Cerullo, M. Zavelani-Rossi, and C. Lienau. Ultrafast optical excitations of metallic nanostructures: from light confinement to a novel electron source. New J. Phys., 9(10):397-397, 2007. Cited on page 15.

[94] R. R. Freeman, T. J. McIlrath, P. H. Bucksbaum, and M. Bashkansky. Pondermotive effects on angular distributions of photoelectrons. Phys. Rev. Lett., 57(25):3156-3159, 1986. Cited on page 15. 
[95] F. Banfi, C. Giannetti, G. Ferrini, G. Galimberti, S. Pagliara, D. Fausti, and F. Parmigiani. Experimental Evidence of Above-Threshold Photoemission in Solids. Phys. Rev. Lett., 94(3):037601, 2005. Cited on page 15.

[96] G. Ferrini, F. Banfi, C. Giannetti, and F. Parmigiani. Non-linear electron photoemission from metals with ultrashort pulses. Nucl. Instruments Methods Phys. Res. Sect. A, 601(1-2):123-131, 2009. Cited on page 15.

[97] H. R. Reiss. Effect of an intense electromagnetic field on a weakly bound system. Phys. Rev. A, 22(5):1786-1813, 1980. Cited on pages 15 and 18.

[98] H. R. Reiss. Energetic electrons in strong-field ionization. Phys. Rev. A, 54(3):R1765R1768, 1996. Cited on page 15.

[99] G Farkas, Z. Gy. Horváth, and I. Kertész. Influence of optical field emission on the nonlinear photoelectric effect induced by ultrashort laser pulses. Phys. Lett. A, 39(3):231-232, 1972. Cited on page 16.

[100] C. Tóth, G. Farkas, and K. L. Vodopyanov. Laser-induced electron emission from an Au surface irradiated by single picosecond pulses at $\lambda=2.94 \mu \mathrm{m}$. The intermediate region between multiphoton and tunneling effects. Appl. Phys. B, 53(4):221225, 1991. Cited on page 16.

[101] L. V. Keldysh. Ionization in the field of a strong electromagnetic wave. Sov. Phys. JETP, 20:1307-1314, 1965. Cited on page 16.

[102] C. C. Neacsu, G. A. Reider, and M. B. Raschke. Second-harmonic generation from nanoscopic metal tips: Symmetry selection rules for single asymmetric nanostructures. Phys. Rev. B, 71(20):201402, 2005. Cited on page 16.

[103] N. Behr and M. B. Raschke. Optical Antenna Properties of Scanning Probe Tips: Plasmonic Light Scattering, Tip-Sample Coupling, and Near-Field Enhancement. J. Phys. Chem. C, 112(10):3766-3773, 2008. Cited on page 16.

[104] Y. C. Martin, H. F. Hamann, and H. K. Wickramasinghe. Strength of the electric field in apertureless near-field optical microscopy. J. Appl. Phys., 89(10):5774, 2001. Cited on page 16.

[105] S. Thomas, M. Krüger, M. Förster, M. Schenk, and P. Hommelhoff. Probing of Optical Near-Fields by Electron Rescattering on the $1 \mathrm{~nm}$ Scale. Nano Lett., 13(10):4790-4794, 2013. Cited on page 16. 
[106] A. Bouhelier, M. Beversluis, A. Hartschuh, and L. Novotny. Near-Field SecondHarmonic Generation Induced by Local Field Enhancement. Phys. Rev. Lett., 90(1):013903, 2003. Cited on pages 16 and 76.

[107] M. K. Miller, A. Cerezo, M. G. Hetherington, and G. D. W. Smith. Atom Probe Field Ion Microscopy. Oxford University Press, 1996. Cited on page 16.

[108] C. Nowak, R. Kirchheim, and G. Schmitz. Electric-field-induced low temperature oxidation of tungsten nanowires. Appl. Phys. Lett., 89(14):143104, 2006. Cited on page 17 .

[109] G. Herink, D. R. Solli, M. Gulde, R. Bormann, and C. Ropers. Strong-Field Photoemission from Metallic Nanotips. Prog. Nonlinear Nano-Optics. Springer International Publishing, 2014. Cited on page 18.

[110] J. Güdde, J. Hohlfeld, J. G. Müller, and E. Matthias. Damage threshold dependence on electron-phonon coupling in Au and Ni films. Appl. Surf. Sci., 127129(1-2):40-45, 1998. Cited on page 18.

[111] S. A. Hilbert, A. Neukirch, C. J. G. J. Uiterwaal, and H. Batelaan. Exploring temporal and rate limits of laser-induced electron emission. J. Phys. B, 42(14):141001, 2009. Cited on page 18.

[112] F. Krausz and M. Ivanov. Attosecond physics. Rev. Mod. Phys., 81(1):163-234, 2009. Cited on page 18.

[113] D. Shorokhov and A. H. Zewail. 4D electron imaging: principles and perspectives. Phys. Chem. Chem. Phys., 10(20):2879-2893, 2008. Cited on pages 19 and 20.

[114] A. Gahlmann, S. Tae Park, and A. H. Zewail. Ultrashort electron pulses for diffraction, crystallography and microscopy: theoretical and experimental resolutions. Phys. Chem. Chem. Phys., 10(20):2894, 2008. Cited on pages 19 and 20.

[115] A. H. Zewail. 4D Ultrafast Electron Diffraction, Crystallography, and Microscopy. Annu. Rev. Phys. Chem., 57(1):65-103, 2006. Cited on pages 19 and 20.

[116] J. Hoffrogge, J. Paul Stein, M. Krüger, M. Förster, J. Hammer, D. Ehberger, P. Baum, and P. Hommelhoff. Tip-based source of femtosecond electron pulses at 30 keV. J. Appl. Phys., 115(9):094506, 2014. Cited on page 19. 
[117] B. J. Claessens, S. B. van der Geer, G. Taban, E. J. D. Vredenbregt, and O. J. Luiten. Ultracold Electron Source. Phys. Rev. Lett., 95(16):164801, 2005. Cited on page 19 .

[118] A. J. McCulloch, D. V. Sheludko, S. D. Saliba, S. C. Bell, M. Junker, K. A. Nugent, and R. E. Scholten. Arbitrarily shaped high-coherence electron bunches from cold atoms. Nat. Phys., 7(10):785-788, 2011. Cited on page 19.

[119] S. B. van der Geer, M. J. de Loos, E. J. D. Vredenbregt, and O. J. Luiten. Ultracold Electron Source for Single-Shot, Ultrafast Electron Diffraction. Microsc. Microanal., 15(04):282-289, 2009. Cited on page 19.

[120] T. van Oudheusden, P. L. E. M. Pasmans, S. B. van der Geer, M. J. de Loos, M. J. van der Wiel, and O. J. Luiten. Compression of Subrelativistic Space-ChargeDominated Electron Bunches for Single-Shot Femtosecond Electron Diffraction. Phys. Rev. Lett., 105(26):264801, 2010. Cited on pages 19 and 87.

[121] M. Gao, H. Jean-Ruel, R. R. Cooney, J. Stampe, M. de Jong, M. Harb, G. Sciaini, G. Moriena, and R. J. D. Miller. Full characterization of RF compressed femtosecond electron pulses using ponderomotive scattering. Opt. Express, 20(11):12048, 2012. Cited on pages 19 and 87.

[122] F. Fu, S. Liu, P. Zhu, D. Xiang, J. Zhang, and J. Cao. High quality single shot ultrafast $\mathrm{MeV}$ electron diffraction from a photocathode radio-frequency gun. Rev. Sci. Instrum., 85(8):083701, 2014. Cited on page 19.

[123] S. P. Weathersby, G. Brown, M. Centurion, T. F. Chase, R. Coffee, J. Corbett, J. P. Eichner, J. C. Frisch, A. R. Fry, M. Gühr, N. Hartmann, C. Hast, R. Hettel, R. K. Jobe, E. N. Jongewaard, J. R. Lewandowski, R. K. Li, A. M. Lindenberg, I. Makasyuk, J. E. May, D. McCormick, M. N. Nguyen, A. H. Reid, X. Shen, K. Sokolowski-Tinten, T. Vecchione, S. L. Vetter, J. Wu, J. Yang, H. A. Dürr, and X. J. Wang. Mega-electron-volt ultrafast electron diffraction at SLAC National Accelerator Laboratory. Rev. Sci. Instrum., 86(7):073702, 2015. Cited on page 19.

[124] M. R. Armstrong, K. Boyden, N. D. Browning, G. H. Campbell, J. D. Colvin, W. J. DeHope, A. M. Frank, D. J. Gibson, F. Hartemann, J. S. Kim, W. E. King, T. B. LaGrange, B. J. Pyke, B. W. Reed, R. M. Shuttlesworth, B. C. Stuart, and B. R. Torralva. Practical considerations for high spatial and temporal resolution 
dynamic transmission electron microscopy. Ultramicroscopy, 107(4-5):356-367, 2007. Cited on page 20.

[125] C. Gerbig, A. Senftleben, S. Morgenstern, C. Sarpe, and T. Baumert. Spatiotemporal resolution studies on a highly compact ultrafast electron diffractometer. New J. Phys., 17(4):043050, 2015. Cited on page 20.

[126] H. Boersch. Experimentelle Bestimmung der Energieverteilung in thermisch ausgelösten Elektronenstrahlen. Zeitschrift für Phys., 139(2):115-146, 1954. Cited on page 20 .

[127] M. Reiser. Theory and Design of Charged Particle Beams. Wiley, 2008. Cited on pages 21, 22, 23 and 24 .

[128] H. Rose. Geometrical Charged-Particle Optics. 2nd edition, Springer, 2012. Cited on pages 22,23 and 87 .

[129] F. J. Sacherer. RMS Envelope Equations with Space Charge. IEEE Trans. Nucl. Sci., 18(3):1105-1107, 1971. Cited on page 22.

[130] W. Lauterborn and T. Kurz. Coherent Optics. 2nd edition, Springer Berlin Heidelberg New York, 2003. Cited on pages 24, 25 and 26.

[131] E. Abbe. Beiträge zur Theorie des Mikroskops und der mikroskopischen Wahrnehmung. Arch. für mikroskopische Anat., 9(1):456-468, 1873. Cited on page 27.

[132] I. M. Watt. The princples and practice of electron microscopy. Cambridge University Press, 1989. Cited on pages 28 and 29.

[133] S. Horiuchi. Fundamentals of High-Resolution Transmission Electron Microscopy. Elsevier Science B.V., 1994. Cited on pages 28, 31, 52 and 97.

[134] C. Kisielowski, B. Freitag, M. Bischoff, H. van Lin, S. Lazar, G. Knippels, P. Tiemeijer, M. van der Stam, S. von Harrach, M. Stekelenburg, M. Haider, S. Uhlemann, H. Müller, P. Hartel, B. Kabius, D. Miller, I. Petrov, E. A. Olson, T. Donchev, E. A. Kenik, A. R. Lupini, J. Bentley, S. J. Pennycook, I. M. Anderson, A. M. Minor, A. K. Schmid, T. Duden, V. Radmilovic, Q. M. Ramasse, M. Watanabe, R. Erni, E. A. Stach, P. Denes, and U. Dahmen. Detection of Single Atoms and Buried Defects in Three Dimensions by Aberration-Corrected Electron Microscope with 0.5-ÅInformation Limit. Microsc. Microanal., 14(05):469477, 2008. Cited on page 28. 
[135] B. A. Szabo and I. Babuška. Finite Element Analysis. Wiley, 1991. Cited on pages 35 and 36 .

[136] J.-M. Jin. The Finite Element Method in Electromagnetics. Wiley, 2014. Cited on pages 35 and 36 .

[137] C. Johnson. Numerical Solution of Partial Differential Equations by the Finite Element Method. Courier Corporation, 2012. Cited on page 36.

[138] S. Strauch. Tip-based photoelectron sources for ultrafast transmission electron microscopy. Master Thesis. Georg-August-Universität Göttingen, 2013. Cited on pages 38,39 and 42 .

[139] H. Yanagisawa, M. Hengsberger, D. Leuenberger, M. Klöckner, C. Hafner, T. Greber, and J. Osterwalder. Energy Distribution Curves of Ultrafast Laser-Induced Field Emission and Their Implications for Electron Dynamics. Phys. Rev. Lett., 107(8):087601, 2011. Cited on pages 39, 64 and 83.

[140] M. Aidelsburger, F. O. Kirchner, F. Krausz, and P. Baum. Single-electron pulses for ultrafast diffraction. Proc. Natl. Acad. Sci., 107(46):19714-19719, 2010. Cited on page 39 .

[141] H. Yanagisawa. Site-selective field emission source by femtosecond laser pulses and its emission mechanism. Ann. Phys., 525(1-2):126-134, 2013. Cited on pages 39 and 64.

[142] S. Schweda. Entwicklung eines Experiments zur zeitaufgelösten Beugung niederenergetischer Elektronen. Master Thesis. Georg-August-Universität Göttingen, 2013. Cited on page 42 .

[143] P. J. Bryant, H. S. Kim, Y. C. Zheng, and R. Yang. Technique for shaping scanning tunneling microscope tips. Rev. Sci. Instrum., 58(6):1115, 1987. Cited on page 46 .

[144] M. Gulde. Optisch induzierte Feldemission an metallischen Nanostrukturen. Diploma Thesis. Georg-August-Universität Göttingen, 2010. Cited on pages 52, 53 and 94.

[145] R. Bormann. Lokalisierte Femtosekunden-Photoemission aus nanometrischen Goldspitzen. Diploma Thesis. Georg-August-Universität Göttingen, 2010. Cited on pages 52,53 and 94 . 
[146] J. G. Fujimoto, J. M. Liu, E. P. Ippen, and N. Bloembergen. Femtosecond Laser Interaction with Metallic Tungsten and Nonequilibrium Electron and Lattice Temperatures. Phys. Rev. Lett., 53(19):1837-1840, 1984. Cited on pages 53 and 86.

[147] G. Herink. Developments in Femtosecond Nanoelectronics - Ultrafast Emission and Control of Electrins in Optical Near-Fields. PhD thesis. Georg-AugustUniversität Göttingen, 2014. Cited on pages 53, 88 and 103.

[148] S. Passlack, S. Mathias, O. Andreyev, D. Mittnacht, M. Aeschlimann, and M. Bauer. Space charge effects in photoemission with a low repetition, high intensity femtosecond laser source. J. Appl. Phys., 100(2):024912, 2006. Cited on pages 53 and 94 .

[149] E. W. Müller. Work Function of Tungsten Single Crystal Planes Measured by the Field Emission Microscope. J. Appl. Phys., 26(6):732, 1955. Cited on page 56.

[150] G. F. Smith. Thermionic and Surface Properties of Tungsten Crystals. Phys. Rev., 94(2):295-308, 1954. Cited on page 56.

[151] H. Yanagisawa, C. Hafner, P. Doná, M. Klöckner, D. Leuenberger, T. Greber, J. Osterwalder, and M. Hengsberger. Laser-induced field emission from a tungsten tip: Optical control of emission sites and the emission process. Phys. Rev. B, 81(11):115429, 2010. Cited on page 64.

[152] F. Yang, J. R. Sambles, and G. W. Bradberry. Long-range surface modes supported by thin films. Phys. Rev. B, 44(11):5855-5872, 1991. Cited on page 65 .

[153] J. Zenneck. Über die Fortpflanzung ebener elektromagnetischer Wellen längs einer ebenen Leiterfläche und ihre Beziehung zur drahtlosen Telegraphie. Ann. Phys., 328(10):846-866, 1907. Cited on page 65.

[154] I. H. Maltison. Refraction and Dispersion of Synthetic Sapphire. J. Opt. Soc. Am., 52(12):1377, 1962. Cited on page 74 .

[155] Z. Lin, L. V. Zhigilei, and V. Celli. Electron-phonon coupling and electron heat capacity of metals under conditions of strong electron-phonon nonequilibrium. Phys. Rev. B, 77(7):075133, 2008. Cited on page 83.

[156] D. Wytrykus, M. Centurion, P. Reckenthaeler, F. Krausz, a. Apolonski, and E. Fill. Ultrashort pulse electron gun with a MHz repetition rate. Appl. Phys. B, 96(2-3):309-314, 2009. Cited on page 83. 
[157] R. Hackam. Comparison between $50 \mathrm{~Hz}$ AC and DC vacuum breakdown. J. Phys. D, 8(4):L53-L55, 1975. Cited on page 87.

[158] G. H. Kassier, N. Erasmus, K. Haupt, I. Boshoff, R. Siegmund, S. M. M. Coelho, and H. Schwoerer. Photo-triggered pulsed cavity compressor for bright electron bunches in ultrafast electron diffraction. Appl. Phys. B, 109(2):249-257, 2012. Cited on page 87.

[159] W. Probst, G. Benner, J. Bihr, and E. Weimer. An "omega" energy filtering TEM - Principles and applications. Adv. Mater., 5(4):297-300, 1993. Cited on page 87.

[160] F. Hofer, W. Grogger, P. Warbichler, and I. Papst. Quantitative Energy-Filtering Transmission Electron Microscopy (EFTEM). Microchim. Acta, 132(2-4):273288, 1999. Cited on page 87.

[161] M. J. Nobes and M. G. McLaren. Some basic considerations of sector magnets. Proc. 11th Int. Conf. Ion Implant. Technol. IEEE, 1996, pp. 379-382. Cited on page 87 .

[162] G. Herink, L. Wimmer, and C. Ropers. Field emission at terahertz frequencies: AC-tunneling and ultrafast carrier dynamics. New J. Phys., 16(12):123005, 2014. Cited on pages 88 and 103.

[163] L. Wimmer, G. Herink, D. R. Solli, S. V. Yalunin, K. E. Echternkamp, and C. Ropers. Terahertz control of nanotip photoemission. Nat. Phys., 10(6):432-436, 2014. Cited on pages 88 and 103.

[164] G. F. Mancini, B. Mansart, S. Pagano, B. van der Geer, M. de Loos, and F. Carbone. Design and implementation of a flexible beamline for fs electron diffraction experiments. Nucl. Instruments Methods Phys. Res. Sect. A, 691:113-122, 2012. Cited on page 94 .

[165] H. S. Park, J. S. Baskin, O.-H. Kwon, and A. H. Zewail. Atomic-Scale Imaging in Real and Energy Space Developed in Ultrafast Electron Microscopy. Nano Lett., 7(9):2545-2551, 2007. Cited on page 95.

[166] O.-H. Kwon, B. Barwick, H. S. Park, J. S. Baskin, and A. H. Zewail. 4D visualization of embryonic, structural crystallization by single-pulse microscopy. Proc. Natl. Acad. Sci., 105(25):8519-8524, 2008. Cited on page 95. 
[167] V. A. Lobastov, R. Srinivasan, and A. H. Zewail. Four-dimensional ultrafast electron microscopy. Proc. Natl. Acad. Sci., 102(20):7069-7073, 2005. Cited on page 95.

[168] M. S. Grinolds, V. A. Lobastov, J. Weissenrieder, and A. H. Zewail. Fourdimensional ultrafast electron microscopy of phase transitions. Proc. Natl. Acad. Sci., 103(49):18427-18431, 2006. Cited on page 95.

[169] G. H. Campbell, T. LaGrange, J. S. Kim, B. W. Reed, and N. D. Browning. Quantifying transient states in materials with the dynamic transmission electron microscope. J. Electron Microsc. (Tokyo), 59(S1):S67-S74, 2010. Cited on page 96.

[170] B. Barwick, D. J. Flannigan, and A. H. Zewail. Photon-induced near-field electron microscopy. Nature, 462(7275):902-906, 2009. Cited on page 101.

[171] S. T. Park, M. Lin, and A. H. Zewail. Photon-induced near-field electron microscopy (PINEM): theoretical and experimental. New J. Phys., 12(12):123028, 2010. Cited on page 101.

[172] D. A. Plemmons, S. Tae Park, A. H. Zewail, and D. J. Flannigan. Characterization of fast photoelectron packets in weak and strong laser fields in ultrafast electron microscopy. Ultramicroscopy, 146:97-102, 2014. Cited on page 101.

[173] S. T. Park, O.-H. Kwon, and A. H. Zewail. Chirped imaging pulses in fourdimensional electron microscopy: femtosecond pulsed hole burning. New J. Phys., 14(5):053046, 2012. Cited on page 101.

[174] B. Cook, M. Bronsgeest, K. Hagen, and P. Kruit. Improving the energy spread and brightness of thermal-field (Schottky) emitters with PHAST - PHoto Assisted Schottky Tip. Ultramicroscopy, 109(5):403-412, 2009. Cited on page 101.

[175] D. Ehberger, J. Hammer, M. Eisele, M. Krüger, J. Noe, A. Högele, and P. Hommelhoff. Highly Coherent Electron Beam from a Laser-Triggered Tungsten Needle Tip. Phys. Rev. Lett., 114(22):227601, 2015. Cited on page 102.

[176] A. H. V. van Veen, C. W. Hagen, J. E. Barth, and P. Kruit. Reduced brightness of the ZrO/W Schottky electron emitter. J. Vac. Sci. Technol. B, 19(6):2038, 2001. Cited on page 102. 
[177] J. Vogelsang, J. Robin, B. J. Nagy, P. Dombi, D. Rosenkranz, M. Schiek, P. Groß, and C. Lienau. Ultrafast Electron Emission from a Sharp Metal Nanotaper Driven by Adiabatic Nanofocusing of Surface Plasmons. Nano Lett., 15(7):46854691, 2015. Cited on page 103.

[178] B. Schröder, M. Sivis, R. Bormann, S. Schäfer, and C. Ropers. An ultrafast nanotip electron gun triggered by grating-coupled surface plasmons. submitted for review. 2015. Cited on page 103. 


\section{Publications and Conferences}

\section{Publications related to this work}

- R. Bormann, S. Strauch, S. Schäfer, and C. Ropers, An ultrafast electron microscope gun driven by two-photon photoemission from a nanotip cathode, J. Appl. Phys. 118, 173105 (2015)

- B. Schröder, M. Sivis, R. Bormann, S. Schäfer, and C. Ropers An ultrafast nanotip electron gun triggered by grating-coupled surface plasmons, Appl. Phys. Lett. 107, 231105 (2015)

- G. Herink, D. R. Solli, M. Gulde, R. Bormann, C. Ropers, Strong-Field Photoemission from Metallic Nanotips, Progress in Nonlinear Nano-Optics, Springer International Publishing (2014).

- G. Herink, D. R. Solli, L. Wimmer, M. Gulde, K. Echternkamp, S. V. Yalunin, R. Bormann, C. Ropers, Photoemission at metallic nanostructures: multiphoton and strong-field dynamics, SPIE 8623, 862311 (2013).

- R. Bormann, M. Gulde, A. Weismann, S. V. Yalunin, and C. Ropers, Tip-enhanced strong-field photoemission, Phys. Rev. Lett. 105, 147601 (2010).

- M. Gulde, R. Bormann, A. Weismann, S. Yalunin, and C. Ropers, Confined electron emission with femtosecond timing: nonlinearity, localization, enhancement, SPIE 7600, 76001I (2010). 


\section{Parts of this work have been presented on the following conferences}

- 27th International Vacuum Nanoelectronics Conference, Engelberg, Switzerland (2014)

- 10th International Vacuum Electron Sources Conference, Saint-Petersburg, Russia (2014)

- DPG-Frühjahrstagung, Regensburg, Germany (2013) 


\section{Acknowledgments}

Hiermit möchte ich mich bei allen bedanken, die mich während meiner Promotionszeit unterstützt, mir geholfen oder einfach nur mein Leben bereichert haben.

Mein spezieller Dank geht an Claus Ropers, der mich über die Jahre hinweg stets gefördert und gefordert hat. Im Besonderen bin ich ihm dafür dankbar, dass er jederzeit offen für wissenschaftliche Diskussionen und Anregungen war und eine einzigartige Atmosphäre in der Arbeitsgruppe zu schaffen wusste. So wird mir neben dem physikalischen auch das soziale, gruppeninterne Engagement stets in guter Erinnerung bleiben.

Für eine ebenso ausgezeichnete Betreuung, sowohl mit umfangreichem Wissen, als auch tatkräftiger Laborarbeit, möchte ich mich bei Sascha Schäfer bedanken.

Bei Markus Münzenberg möchte ich mich für die freundliche Bereitschaft, die Korreferenz zu übernehmen, herzlich bedanken.

Der Arbeitsgruppe danke ich für die tolle Atmosphäre, sowohl im wissenschaftlichen, als auch im zwischenmenschlichen Bereich. Die Gruppenausflüge und -aktivitäten waren immer eine Freude. Hervorheben möchte ich an dieser Stelle insbesondere die langjährigen Kollegen Max Gulde, Murat Sivis, Georg Herink und Sergey Zayko. Beim gesamten IV. Physikalischen Institut möchte ich mich für die verschiedensten Dinge bedanken, insbesondere aber für den angenehmen und lockeren Umgang.

Desweiteren danke ich meinen Freunden für eine abwechslungsreiche, lustige und schöne Zeit neben der Physik, insbesondere meinen langjährigen Mitbewohnern Christian, Hajo und Carla.

Zuletzt möchte ich mich noch bei meiner Familie bedanken, die mich stets unterstützt hat und immer für mich da war. 
